\title{
WestVirginiaUniversity
}

THE RESEARCH REPOSITORY @ WVU

Graduate Theses, Dissertations, and Problem Reports

2007

\section{Design and real-time control of shipboard power system testbed}

\author{
Pradeep Pant \\ West Virginia University
}

Follow this and additional works at: https://researchrepository.wvu.edu/etd

\section{Recommended Citation}

Pant, Pradeep, "Design and real-time control of shipboard power system testbed" (2007). Graduate Theses, Dissertations, and Problem Reports. 1862.

https://researchrepository.wvu.edu/etd/1862

This Thesis is protected by copyright and/or related rights. It has been brought to you by the The Research Repository @ WVU with permission from the rights-holder(s). You are free to use this Thesis in any way that is permitted by the copyright and related rights legislation that applies to your use. For other uses you must obtain permission from the rights-holder(s) directly, unless additional rights are indicated by a Creative Commons license in the record and/ or on the work itself. This Thesis has been accepted for inclusion in WVU Graduate Theses, Dissertations, and Problem Reports collection by an authorized administrator of The Research Repository @ WVU. For more information, please contact researchrepository@mail.wvu.edu. 


\title{
Design and Real-Time Control of Shipboard Power System Testbed
}

\author{
By \\ Pradeep Pant \\ Thesis submitted to the \\ College of Engineering and Mineral Resources \\ at West Virginia University \\ in partial fulfillment of the requirements \\ for the degree of \\ Master of Science \\ in \\ Electrical Engineering \\ Karl Schoder, Ph.D. \\ Powsiri Klinkhachorn, Ph.D. \\ Ali Feliachi, Ph.D., Chair
}

Lane Department of Computer Science and Electrical Engineering

Morgantown, West Virginia

2007

Keywords: shipboard power system, hardware prototype, real-time control, reconfigurable power system, agent communication, controller area network, power conversion, power electronics.

Copyright 2007 Pradeep Pant 


\begin{abstract}
Design and Real-Time Control of Shipboard Power System Testbed

by

Pradeep Pant

Master of Science in Electrical Engineering

West Virginia University

Professor Ali Feliachi, Ph.D., Chair
\end{abstract}

The objective of this thesis is to design and test a small scale testbed for the all-electric shipboard power distribution system. Shipboard power system is increasingly becoming more reconfigurable, and multi-agent systems are developed to automate routine operation and emergency reconfiguration. Underlying algorithms of these systems have been verified using software simulation tools. However, these simulators run in soft realtime by using simple mathematical models to represent the physical system. These models do not incorporate every aspect of the physical system. A testbed provides a cost effective physical environment to verify these algorithms and control techniques in the real world. This testbed, based on the Navy's notional all electric ship, keeps characteristic features of the Office of Naval Research's Integrated Power System. It provides a platform for testing local and distributed controls. Local embedded controllers on the testbed run in hard real-time, and a CAN bus builds the communication networking among them. Performance of the controllers has been verified successfully, and the platform provides an environment that allows prototyping and testing agent-based higher-level controls and decision making entities. 
This thesis is dedicated to my mother Rid Kumari Panta. 


\section{Acknowledgement}

I would like to thank my advisor, Dr. Ali Feliachi, for providing me an opportunity to work with him. His encouragement and support has been most valuable to me throughout my research work and studies. I am thankful to Dr. Karl Schoder for working very closely to guide me. I would also like to thank Dr. Powsiri Klinkhachorn for his continued guidance and valuable suggestions. I also express my thanks to faculty members and students at APERC for their help and support.

I am thankful to my mother for her love and encouragement. I also thank my wife Neeru for being a great source of encouragement and support. I would like to thank my brothers, especially Deepak for his extraordinary help during my undergraduate and graduate studies. I would not be here without his support. I would like to thank my kids and rest of my family for their support.

This research is sponsored in part by a grant from the US DEPSCoR and ONR (DOD/ONR N000 14-031-0660). 


\section{Table of Contents}

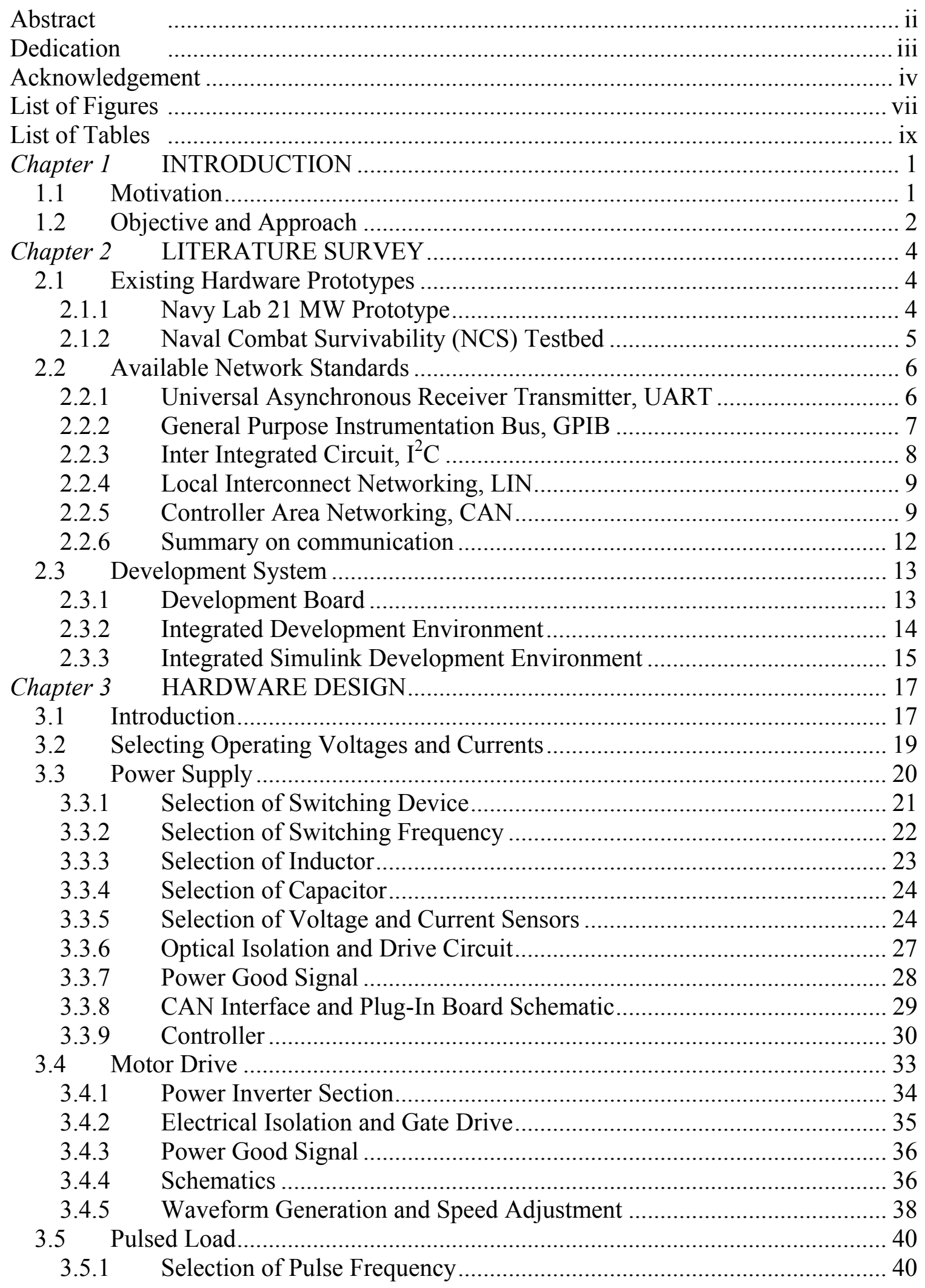




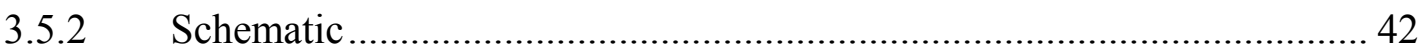

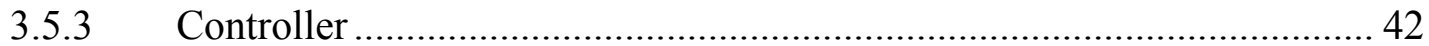

3.6 Shipboard Service Converter Module............................................................. 43

3.6.1 Selection of Output Filter Elements and Sensors ...................................... 44

3.6.2 Circuit Breaker............................................................................... 44

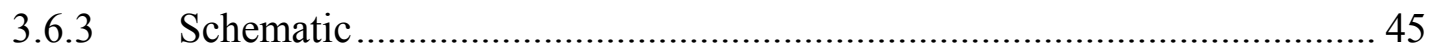

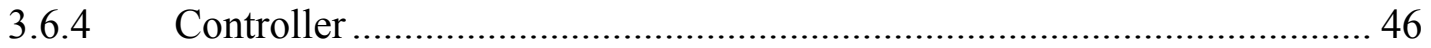

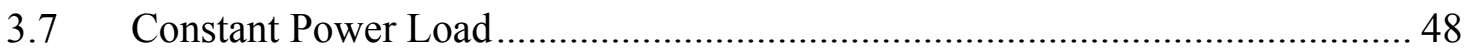

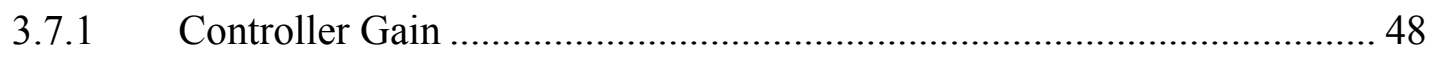

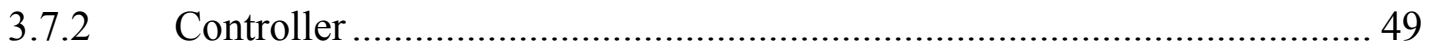

3.8 Internal Power Supply for Sensors and Drives .............................................. 50

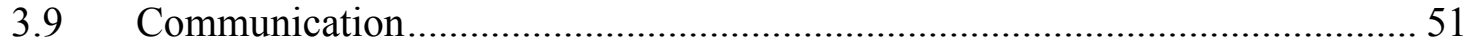

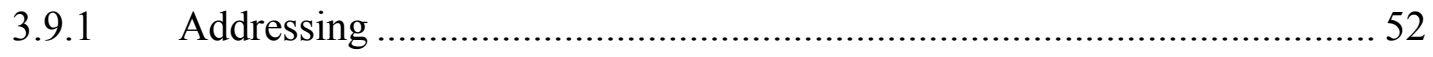

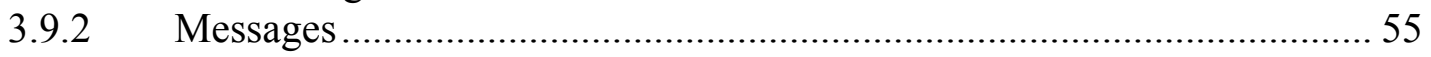

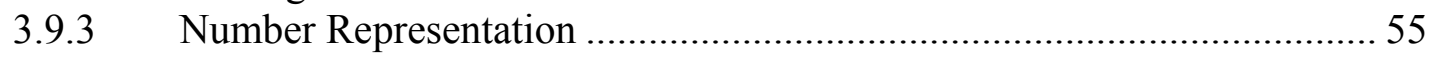

3.10 User Interface for Testbed Monitoring ……….......................................... 59

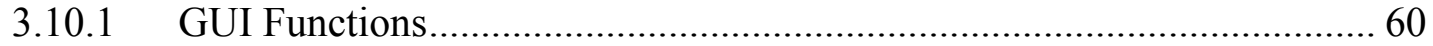

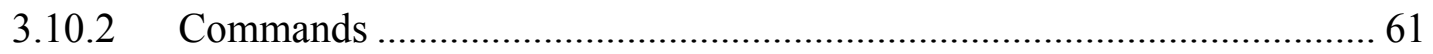

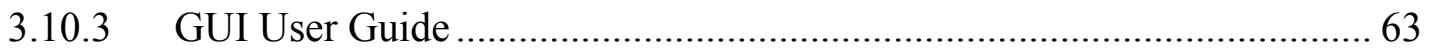

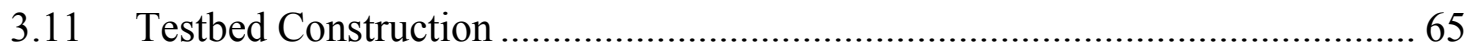

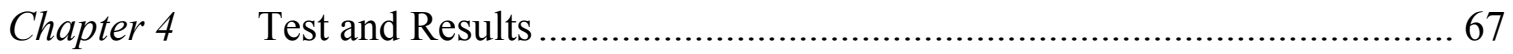

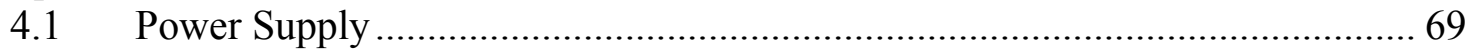

4.2 Shipboard Service Converter Module.............................................................. 71

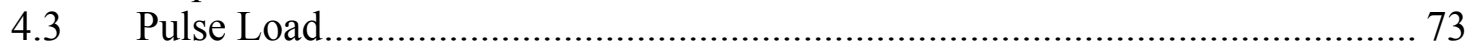

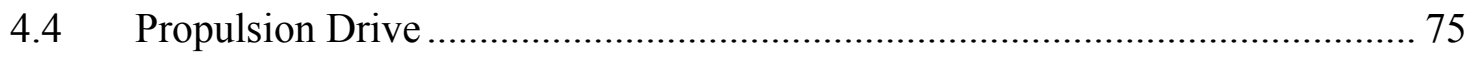

4.5 Constant Power Load............................................................................ 76

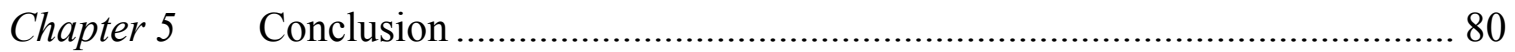

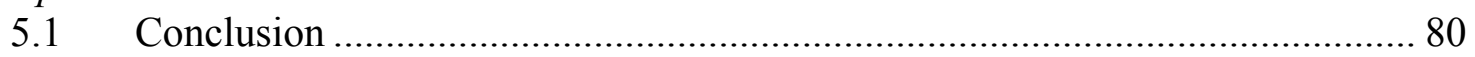

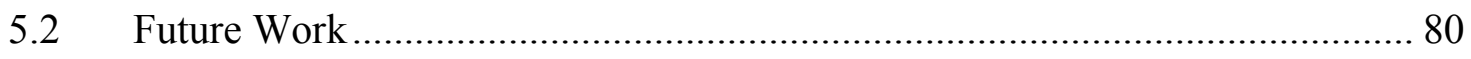

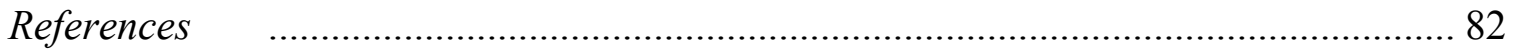

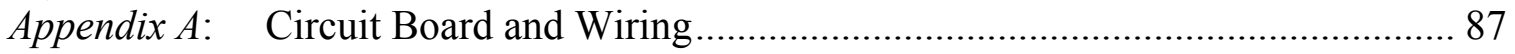




\section{List of Figures}

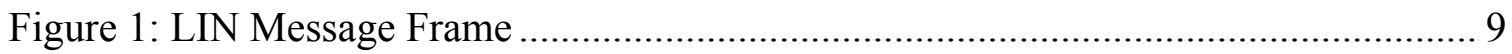

Figure 2: CAN Message Frame …………............................................................ 10

Figure 3: PCAN-USB and DB9 Connector ……………......................................... 12

Figure 4: MC9S12C32 Microcontroller Overview.................................................... 13

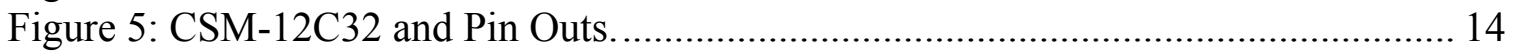

Figure 6: Code Generation Process Flowchart. ......................................................... 15

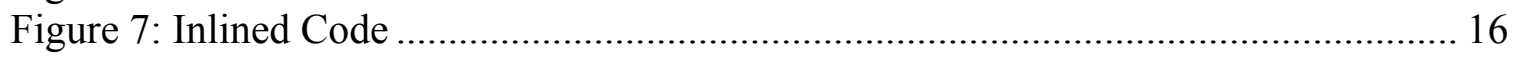

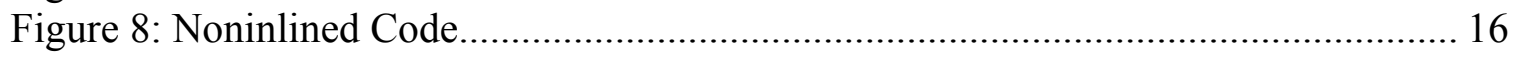

Figure 9: Testbed Components ........................................................................... 18

Figure 10: Voltage Assignment and Estimated Current .................................................. 20

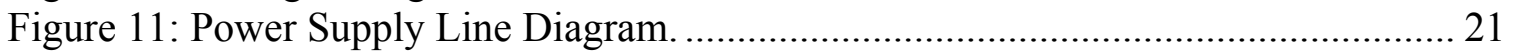

Figure 12: Hall Effect Voltage Sensor...................................................................... 25

Figure 13: Hall Effect Current Sensor. ………………......................................... 26

Figure 14: MOSFET Gate Driver Turned-On and Turned-Off Mode of Operation......... 27

Figure 15: Schematic of MCU Plug-in Board and CAN Driver for Power Supply ......... 29

Figure 16: Detailed Schematic of Power Supply.......................................................... 30

Figure 17: Simulink Model for Power Supply Controller................................................. 32

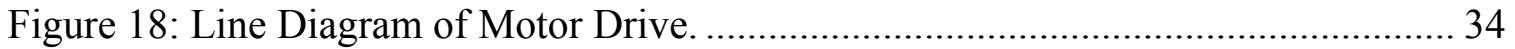

Figure 19: Integrated Power Module............................................................................... 34

Figure 20: Bootstrap Diode Arranged for High Side MOSFET Driving........................... 35

Figure 21: Dead Time Generator for Complementary IGBT …………………............. 36

Figure 22: Detailed Schematic of MC9S12C32 Plug-in Board for Motor Drive............. 37

Figure 23: Detailed Schematic of Motor Drive. …………………………………….... 37

Figure 24: Simulink Model of Motor Drive Controller..................................................... 39

Figure 25: Line Diagram of Pulsed Load. .................................................................. 40

Figure 26: MC9S12C32 Plug-in Board for Pulse Load.................................................. 42

Figure 27: Detailed Schematic of Pulsed Power Load. ………....................................... 42

Figure 28: Simulink Model of Pulse Generator for Pulsed Load. ..................................... 43

Figure 29: Line Diagram of Shipboard Service Converter Module. ................................. 43

Figure 30: Detailed Schematic of Circuit Breaker............................................................ 44

Figure 31: Detailed Schematic of MC9S12C32 Plug-in Board for SSCM. ..................... 45

Figure 32: Detailed Schematic of Shipboard Service Converter Module.......................... 46

Figure 33: Simulink Model of Shipboard Service Converter Module............................... 47

Figure 34: Constant Power Load Controller................................................................ 48

Figure 35: Constant Power Load Controller Simulink Model.......................................... 49

Figure 36: Power Supply for Gate Drives and Relays.................................................... 50

Figure 37: Power Supply for Sensor and MCU ......................................................... 50

Figure 38: CAN Message Format Using Sender's and Receiver's Address. ................... 52

Figure 39: Addresses and Command Summary............................................................ 54

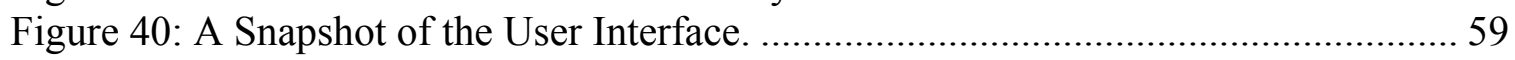

Figure 41: Initialization of Variables and Timer Function for GUI ................................. 60

Figure 42: Initialization Function for Periodic CAN Messaging....................................... 61

Figure 43: GUI Function for Turning "On” and "Off” Entities on the Testbed ............... 61

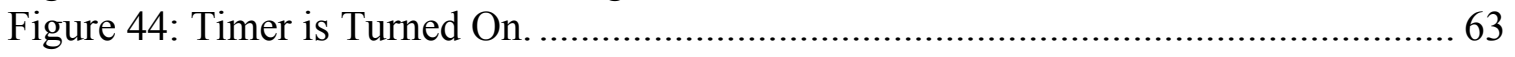




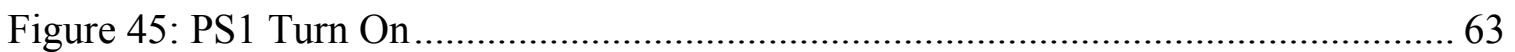

Figure 46: PS1, Breaker R1, and SSCM1 are Turned On. ............................................. 64

Figure 47: GUI Interface for Constant Power Load. ...................................................... 64

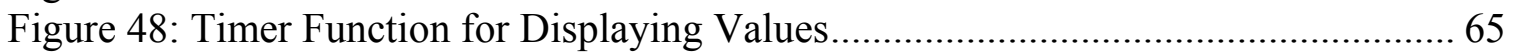

Figure 49: Completely Assembled Testbed Integrated with RA and CCC...................... 66

Figure 50: Test Setup Using Power Analyzer PZ4000 ………...................................... 67

Figure 51: Top View of Testbed and Arrangement for Tests and Demonstration. .......... 68

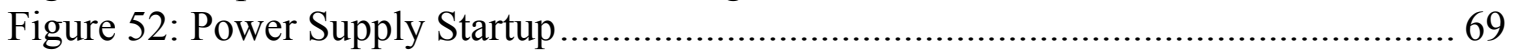

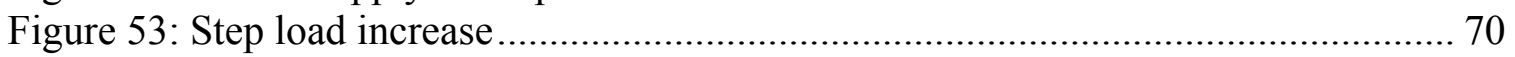

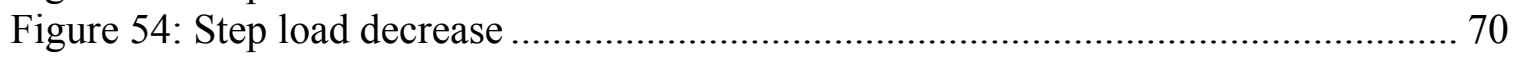

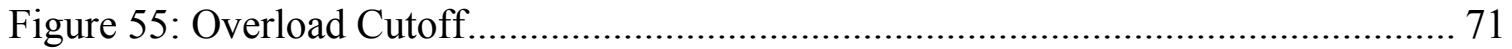

Figure 56: Shipboard Service Converter Module Startup.............................................. 72

Figure 57: Shipboard Service Converter Module Trun-on ............................................. 72

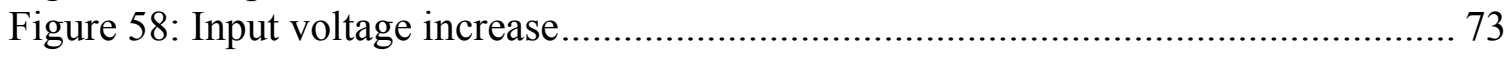

Figure 59: Pulsed Load, Normal Operation.............................................................. 73

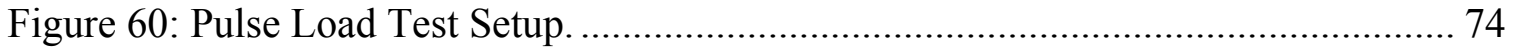

Figure 61: Pulsed Load, Continuous Loading. ........................................................... 74

Figure 62: Test Setup for Motor Drive. ................................................................. 75

Figure 63: $0.27 \mathrm{~Hz}$ Motor Operation. ……………….......................................... 75

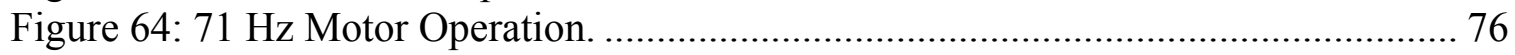

Figure 65: Calculated Voltage Vs Measured Voltage. .................................................. 78

Figure 66: Efficiency at Different Power Levels of CPL.............................................. 78

Figure 67: Constant Power Load with Lower Resistance................................................ 79

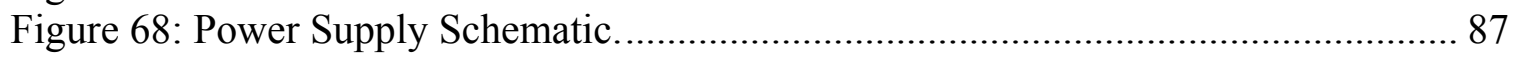

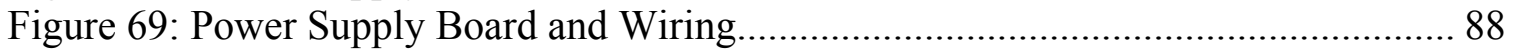

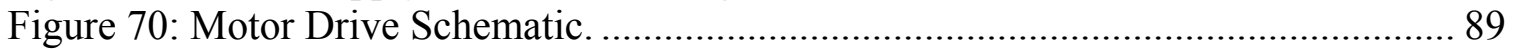

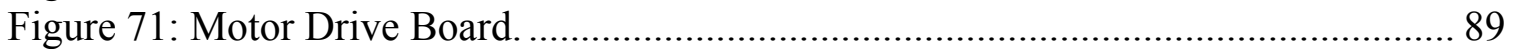

Figure 72: Pulsed Load Schematic. ........................................................................... 90

Figure 73: Pulsed Load Board and Wiring. ................................................................... 90

Figure 74: Shipboard Service Converter Module Schematic. ………………………...... 91

Figure 75: Shipboard Service Converter Module Board. ................................................ 91

Figure 76: Constant Power Load Schematic................................................................. 92

Figure 77: Constant Power Load Circuit Board. ……………………………................ 92 


\section{List of Tables}

Table 1 Maximum Signaling Rates for Various Cable Lengths .................................. 12

Table 2: Clock Speed vs PWM Frequency for MC9S12C32 ...................................... 23

Table 3: Table of Pin Assignment for Power Supply MCU Board. …............................. 29

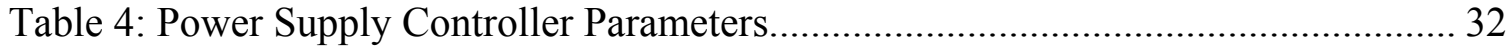

Table 5: Motor Controller Parameters. ....................................................................... 40

Table 6: Shipboard Service Converter Module Controller Parameters. .......................... 47

Table 7: Addresses for Testbed Components. ............................................................ 53

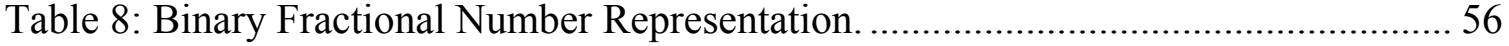

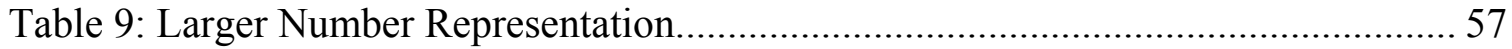

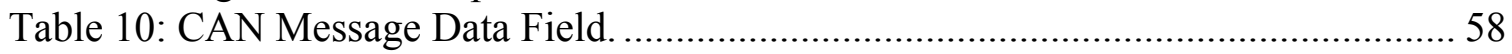

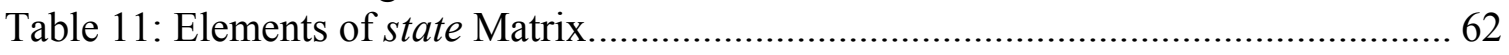

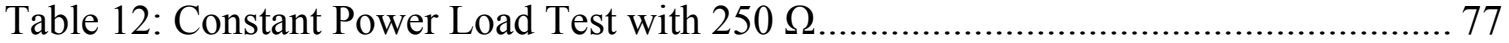




\section{Chapter 1 INTRODUCTION}

The naval ships of the future will use more flexible integrated power systems by adapting electric propulsion motors and electric pulsed high power weapons [33]. The modern all electric shipboards will serve the electric propulsion systems, which typically consume $65 \%$ of the overall load, weaponry systems and general loads including hotel loads, and other appliances. These loads are DC loads or AC loads running from a DC-AC inverter and are arranged in a zonal structure [25]. Generation of the power is usually carried out by gas turbines as prime movers and synchronous generators. These generators provide $\mathrm{AC}$ power, which can be directly used by $\mathrm{AC}$ loads like the propulsion systems. However, distributing electric power through a DC based approach provides new possibilities and flexibilities, and the next generation of ships will build on a DC distribution system incorporating power electronics converter technology for advanced controllability and reconfigurability.

If agent based distributed controls are desired then it will be required that these sensors, actuators, switches and controls be able to share information. Part of the communication infrastructure will have to be able to provide information with real-time constraints to other entities within the system. However, the communication is not the only task these units are required to perform in real-time. Safety features like overload and short circuit protection must also be handled spontaneously in order to prevent further propagation of the effects into the system. Safety related events can most often be handled by power electronics and local controls before exchanging information about the perceived failure.

\subsection{Motivation}

Availability of simulation tools has made the task of testing new concepts easier. Flexibility in software allows quick alternations in the design at less cost. However, a software tool comes with limitations on how closely it can represent a physical system. Software tools like real-time simulators use mathematical models to represent a system and it may be difficult to incorporate every aspect of a physical system into a 
mathematical model. Simulations using higher level platforms or application programs, running on top of general operating systems like Windows or Linux may only provide a soft-real-time environment in which the system can be tested on top of simplified models. Simulation can be considered as first step towards verification as they may need a real life implementation to verify their performance in the real world. In other words, a physical testing or a prototype can be considered as a second step towards verification of a concept.

Optimization of control techniques for local as well as distributed controls help improve survivability and performance for a shipboard power system. A physical hardware that preserves the characteristics of the actual system and provides a scaled down, low cost and easily manageable platform for verifying performance of these new techniques can make the development process more efficient. A platform that can serve both the verification of local as well as the distributed controls requires that the devices be integrated using a communication infrastructure.

\subsection{Objective and Approach}

The objective of this research is to develop a testbed that can provide a validation and demonstration platform for the local and distributed controls of the U.S. Navy's notional electric shipboard power systems. The goal is to build a smaller, scaled platform with respect to power consumption that still preserves the main characteristics of the Naval Combat Survivability (NCS) testbeds, like the propulsion loads, pulsed loads and zonal distribution architecture [43]. The design addresses the following objectives.

- Keep the design and maintenance cost small, without compromising required capabilities.

- $\quad$ Keep testbed size manageable for transportability.

- Add safety features like isolation and protection to keep the system safe for experiments.

- Implement real-time local controls and add communication capabilities to provide a platform for testing distributed controls. 
- Make the testbed reprogrammable for upgrades or implementation of different control techniques.

With the goal of having a complete AC and DC distribution system as featured in the NCS testbed, care must be taken to match voltage, current and settling time of the neighboring devices and controllers to achieve smooth operation of the system. To meet this requirement, devices must be customized as it is difficult to, for example, find matching converters off the shelf. Nevertheless, this project should undertake all the design and implementation of power electronics and controls using off the shelf discrete components.

The rapid growth in semiconductor technology has put several high performance products in the market giving a great spectrum of choices to the designers. These components serve as building blocks for the testbed. The testbed focuses on high performance, low power components and embedded controllers that can provide control and communication. A survey has been conducted to look at different communication techniques to make selection. The following steps were taken to meet the objectives:

- Design power electronics devices to represent elements of the shipboard power system providing one-to-one correspondence with Reconfiguration Agents.

- Implement local controls through programmable embedded devices.

- Provide networked communication interface using Controller Area Networking.

- Develop and implement communication schemes for addressing and messaging.

- Test individual components on the testbed for step responses and efficiencies. 


\section{Chapter 2 LITERATURE SURVEY}

This chapter presents an account of similar work done in shipboard power system prototypes. In the later part of the chapter, a survey of available communication standards and embedded system development tools has been presented.

\subsection{Existing Hardware Prototypes}

Several efforts have been undertaken to build shipboard power system testbeds for verification, demonstration, and educational purposes. These testbeds are built in different sizes and capabilities to fit different goals. A summary on the sizes, topologies, and goals of building some of these testbeds have been presented in this chapter.

\subsubsection{Navy Lab 21 MW Prototype}

This testbed, developed at the Navy's Land Based Engineering Test Site in Philadelphia, is a full scale hardware prototype. This testbed played an important role in the testing of the high-power shipboard components like the PWM drive for the propulsion motor.

Components: The testbed is built around an extremely high power 3-phase induction motor drive. This drive makes use of Insulated Gate Bipolar Transistors (IGBTs) as the switching devices. The capacity of the motor drive is estimated to be 19MW, which is an impressive advance in size when compared to the existing 5MW cycloconverter based motor drives [10]. The testbed has been set up with a $21 \mathrm{MW}$ gas turbine as the prime mover, and the 19MW, $150 \mathrm{RPM}$ induction motor as propulsion system.

Topology: The converter technology uses six pulse-rectifier stages that provide $6 \mathrm{kV}$ to the drive using three separate DC links. The PWM converter consists of multiple identical and synchronously switched IGBT stages, connected in series within each arm.

Purpose: This testbed was intended mainly for testing the high-power high-voltage induction motor drive. This full sized testbed is built without the general shipboard or the zonal loads and hence provides limited scope for the purpose of validation and testing of the overall system [33]. 


\subsubsection{Naval Combat Survivability (NCS) Testbed}

Due to the desire to build more accessible and cost effective testbeds, Naval Combat Survivability Testbed was developed as a joint effort of two universities and in collaboration with the U.S. Navy. This lower voltage and lower power testbed is a valid and less expensive option for many research projects that address controls and reconfiguration concepts.

Components: NCS Testbed is made up of two separate hardware units, which are located at two different universities [51]. The first unit, called the Generation and Propulsion Testbed (GPT), is comprised of a pulse load and a $37 \mathrm{~kW}$ induction motor based propulsion load. GPT is permanently located at Purdue University. It includes a $59 \mathrm{~kW}$ generator unit, which powers the propulsion drive and the pulsed load. The second unit of the NCS testbed is the DC Distribution testbed (DCDT). DCDT is located at the University of Missouri-Rolla and consists of a power supply and the DC zonal loads [47]. DCDT is based on a $15 \mathrm{~kW}$ main power supply and is designed to be transported to and integrated with the GPT testbed. DCDT also consists of a second power supply, which can be programmed as Model-in-Loop (MIL) to simulate devices not available in the lab.

Considering its purpose as research and teaching tool, a lower voltage has been used for the $\mathrm{AC}$ and $\mathrm{DC}$ distribution testbeds. The $\mathrm{AC}$ system uses a nominal voltage of $560 \mathrm{~V}_{\mathrm{L}-\mathrm{L}}$. The propulsion system is built around a $460 \mathrm{~V}_{\mathrm{L}-\mathrm{L}}$ induction motor. Main DC buses carry a voltage of $500 \mathrm{Vdc}$.

Topology: The DC testbed consists of three load zones, powered at the voltage level of 400-420 V. Load zones consist of an inverter based 3-phase load, 3.7kW motor drive, and a $5 \mathrm{~kW}$ constant power load [51].

Power supply and the DC-DC converter modules are designed to step-down voltage levels using buck-converter topology. Constant power loads also uses the same converter topology but controls are intended at regulating power. Propulsion drive and motor control are based on a three phase bridge converter. 
Purpose: The hardware was developed for the purpose of validating new approaches for modeling and controlling naval power distribution and propulsion systems [47][43].

\subsection{Available Network Standards}

Most of the single chip digital controllers these days offer hardware support for communication interfaces. Several different communication standards have been developed with different complexities and capabilities. Some offer high speed while some other offer high reliability or high addressability. These standards have been developed to fit specific needs and cost requirements. For example, Controller Area Network (CAN) offers moderate speed in noisy environments, while Local Interconnect Networking (LIN) offers a lower cost solution with lower speed for similar needs. Media Oriented System Transport (MOST) offers powerful communication interface for transferring large amount of data. Choices with less capabilities, speed, and cost are Inter Integrated Circuit $\left(\mathrm{I}^{2} \mathrm{C}\right)$, Serial Peripheral Interface (SPI), and the Universal Asynchronous Receive Transmit (UART) [11]. Hewlett Packard Instrumentation Bus (HP-IB) offers a parallel data interface at very high speed.

\subsubsection{Universal Asynchronous Receiver Transmitter, UART}

The first UART specification came out as early as 1959 . The early version of this UART was developed by Digital Equipment Corporation for its Programmed Data Processor (PDP-1) computers [5]. Being one of the most basic communication standards, it only offers specifications for the physical layer and is designed for communication between two devices only. It offers a maximum achievable speed of 20kbps.

The UART does not directly generate or receive the external signaling levels (such as voltages on wires) that are used between different equipment. Recommended Standard 232, more commonly known as RS232, is well established communication standard that uses voltage signaling. RS232 offers up to 20kbps speed. TIA/EIA 423 came out as increased speed version of RS232 with a speed of 100kbps. Later TIA/EIA-562 came out as a low voltage version of RS232.

RS232 does not make any specification available with respect to the maximum cable length but it specifies the maximum line capacitance to be less than $2500 \mathrm{pF}$ with a load 
impedance of $7 \mathrm{k} \Omega$. Based on this, an estimate for the maximum length paired/twisted wire is 20 meters.

UART specification defines RS232 as single ended, meaning it is not based on a differential bus voltage, and unidirectional point-to-point interface. This standard is not suited for establishing a communication network.

\subsubsection{General Purpose Instrumentation Bus, GPIB}

In 1960 Hewlett Packard developed a communication bus called HP- Instrumentation Bus, or HPIB, which later was reproduced by other industries renaming it as General Purpose IB. The GPIB is a parallel communication bus except for a few serial communication features used by some control and handshaking lines. It offers a connection speed as high as 1MBytes/s in its original standard. In 2003 the upgrade of IEEE 488.1 standard was released that allows buses to transfer data at speeds as high as 8 MBytes/s [45]. It requires a total of 24 connection lines, which includes bidirectional data lines, handshaking signals, control signals, and ground lines. Device addressing is limited to 15 devices and one must be a master [21][57].

Devices on the network assume one of the following three roles at a time.

1. Controller, which enables/disables devices.

2. Talker, which sends data to another device.

3. Listener, which receives information from Talker.

The devices can keep changing their form over the time. Although devices keep passing control from one to the other there can be only one controller at any given time. The controller makes decision as to which device becomes active, meaning which device talks, which listens, which un-talks and which un-listens. There are some hard-coded functionalities which are not alterable by the controller. A 5 bit BCD is soft coded into the devices to assign its address.

Data bits: The 8 data bits for GPIB are bidirectional in nature. DIO1 through DIO8 data bits are shared by all the devices on the bus. 
Handshaking: 3 bits for handshaking are Data Valid (DAV), Not Ready for Data (NRFD) and Not Data Accepted (NDAC). The active talker controls DAV to inform whether data bits are available or not, while the listener(s) control NRFD and NDAC to let the talker know if the sent data were accepted.

Control: There are 5 control bits which are used for the communication of control, status and error information among the devices. End-Or-Identify (EOI), Interface Clear (IFC), Service Request (SRQ), Attention (ATN) and Remote Enable (REN). ATN indicates whether the value is a data or an interface message.

The total length of all the cables used is less than or equal to 2 meters times the number of devices connected together up to an absolute maximum of 20 meters. For example, if two devices are connected the maximum length is 4 meters but if 11 devices are connected the maximum length is limited to 20 meters [21].

\subsubsection{Inter Integrated Circuit, $I^{2} \mathrm{C}$}

In the early 1980's Philips Semiconductors developed a communication standard targeting serial connection between Central Processing Units and the peripheral chips in TV-sets. This standard interconnects integrated circuit chips through a pair of communication lines.

$\mathrm{I}^{2} \mathrm{C}$ is a synchronous communication standard in which one or more of the connected devices provide clock to the remaining devices in the network. It uses two signal wires, known as Serial Data (SDA) and Serial Clock (SCL), and a ground wire.

Each device connected to the bus carries a unique address. A master-slave relationship exists between the devices at all the times; masters can operate as master-transmitters or as master-receivers and same applies to the slaves [1].

In 1992 Philips released Version 1.0 of the standard, which replaced the previous 7 bit addressing by 10 bits. It also specified the slope control and input filtering standard for fast-mode devices in order to improve Electromagnetic Compatibility (EMC). In 1998, Version 2.0 was released and it offers a hi-speed mode that allows up to 3.4Mbps. The current version is 2.1 and it offers additional auto correction features during failures. 


\subsubsection{Local Interconnect Networking, LIN}

LIN specification was originally developed based on the commonly available UART hardware; this was done deliberately to bring the cost of implementation down [50]. This protocol is based on single master and multiple slaves networking architecture.

LIN version 1.1 was released in 1999 and since then the specification has evolved to version 2.1 [56], which also makes provisions for significant additional diagnostics capabilities.

The LIN-Master uses one or more predefined scheduling tables to start the sending and receiving process. LIN Frame consists of two parts, the header and the response. The header shown in Figure 1 is always sent by the LIN Master, while the response is sent by only one dedicated LIN-Slave.

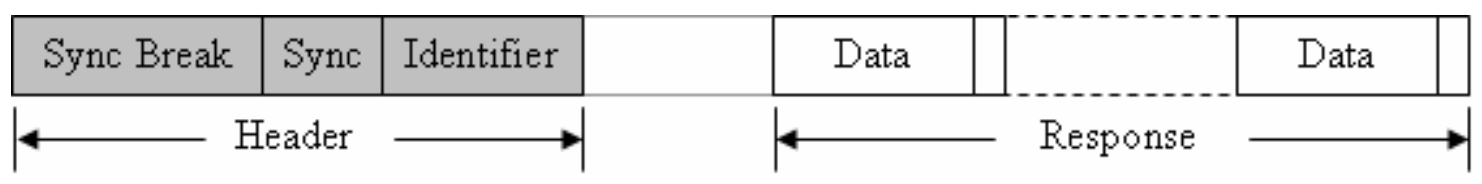

Figure 1: LIN Message Frame

LIN data are transmitted serially as eight bit data bytes with one start and one stop-bit and no parity. The standard defines $20 \mathrm{kbps}$ as the maximum achievable speed.

The baud rates in LIN are adjusted by negotiating there slaves use Sync byte, a sequence of ' 0 's and ' 1 's sent by the master, to calculate and adjust their bit timing and rate.

\subsubsection{Controller Area Networking, CAN}

Controller Area Network is a serial communication standard designed to work in a noisy environment. It supports up to $1 \mathrm{Mbps}$ communication speed over a distance of 40 meters. Maximum distance that can be covered using CAN is 1000 meters. It was designed in Germany during 1980 by a company named Robert Bosch $\mathrm{GmbH}$, mainly for automotive applications [16]. It has found applications in other areas of the industry. Unlike LIN, I2C, SPI and even GPIB, it does not follow master-slave communication architecture, making it more appropriate for agent based communication. CAN standard is equipped with a number of autonomous error detection mechanisms, making it reliable as a networking solution even within noisy environments. In many applications, other smaller networks like LIN, $\mathrm{I}^{2} \mathrm{C}$ and SPI have been used as sub-network for CAN. 
CAN hardware is built on a three-layer architecture and a $4^{\text {th }}$ layer on top of the hardware is the application layer [48]. These layers do not exactly follow the ISO's Open System Interconnection (OSI) model. The top two layers among the three layers are the Object Layer and the Transfer Layer. Looking at ISO/OSI model, these two layers, collectively, can be considered equivalent to the data link layer. The bottom layer is the transfer layer, which performs tasks like message framing, arbitration, and error handling.

The physical layer defines the electrical properties for the actual bit transfer between nodes. This layer defines voltage level for the electrical connection and also the bit timing. CAN physical layer uses a pair of differential lines to transmit messages among the nodes.

Several different higher layer protocols have been developed using CAN as the physical and transportation layer. These application specific protocols have set forward standards like DeviceNet, NMEA 2000, and CANopen. DeviceNet is a scaled down version of ControlNet, which connects higher-level devices such as programmable controllers to the simple industrial devices like sensors and actuators [12]. NMEA 2000 was developed under the initiative of National Marine Electronics Association (NMEA). This standard targets data communication among marine electronic devices such as depth finders, chartplotters, navigation instruments, engines, tank level sensors, and GPS receivers [29]. CANopen was released in 1995 under the chairmanship of Bosch and developed for applications of motion-oriented machine control networks. Its application has expanded to several different areas lately. Currently CAN in Automation (CiA) maintains the protocol and offers specifications for the CAN application layer [8].

\section{Message Format}

An extended CAN frame uses 29bit arbitration, data bytes, and CRC. The breakdown of a CAN frame is shown in Figure 2.

\begin{tabular}{|c|c|c|c|c|c|c|c|c|c|c|c|}
\hline $\begin{array}{l}S \\
0 \\
F\end{array}$ & $\begin{array}{c}\text { 11-BIT } \\
\text { ARBITRATION ID }\end{array}$ & $\begin{array}{l}\mathrm{S} \\
\mathrm{R} \\
\mathrm{R}\end{array}$ & $\mid \begin{array}{l}\mathrm{I} \\
\mathrm{D} \\
\mathrm{E}\end{array}$ & $\begin{array}{c}\text { 18-BIT } \\
\text { ARBITRATION ID }\end{array}$ & $\left|\begin{array}{l}\mathbf{R} \\
\mathrm{T} \\
\mathrm{R}\end{array}\right|$ & $\begin{array}{l}r \\
0\end{array}$ & DLC & $\begin{array}{c}\mathbf{0} \ldots \mathbf{8} \\
\text { BYTES DATA }\end{array}$ & CRC & $\begin{array}{l}\mathrm{A} \\
\mathrm{C} \\
\mathrm{K}\end{array}$ & \\
\hline
\end{tabular}

Figure 2: CAN Message Frame

SOF (start-of-frame) bit -- indicates the beginning of a message with a dominant bit 
Arbitration ID -- identifies the message and indicates the message's priority. Frames come in two formats: standard, which uses 11-bit arbitration ID, and extended, which uses a 29-bit arbitration ID.

SRR (substitute remote request) -- SRR bit is always transmitted as a recessive bit to ensure that, in the case of arbitration between a Standard Data Frame and an Extended Data Frame, the Standard Data Frame will always have priority if both messages have the same base (11 bit) identifier.

IDE (identifier extension) -- This bit allows differentiation between standard and extended frames

RTR (remote transmission request) bit -- serves to differentiate a remote frame from a data frame. A dominant (logic 0) RTR bit indicates a data frame. A recessive (logic 1) RTR bit indicates a remote frame.

DLC (data length code) -- indicates the number of bytes the data field contains

Data Field -- contains 0-8 bytes of data

CRC (cyclic redundancy check) -- contains 15-bit cyclic redundancy check code and a recessive delimiter bit. The CRC field is used for error detection.

ACK (acknowledgement) slot -- any CAN controller that correctly receives the message sends an ACK bit at the end of the message. The transmitting node checks for the presence of the ACK bit on the bus and reattempts transmission if no acknowledgement is detected.

EOF (end-of-frame) bit - marks the end of a message.

\section{Relationship between bus length and baud rate}

The arbitration in CAN communication requires that the front wave of the first bit of the message make a round trip to the end of the network before the bit is designated by the receiver of the sending node as dominant or recessive. The sampling is performed at two thirds of the bit width. If a twisted pair is used the typical propagation time is considered to be 5 ns per meter [52]. Other things to be considered are amplitude loss due to the loss mechanism of the cable and the input impedance of the bus transceiver. An inverse 
relationship exists between the distance and the baud rate of the CAN message. Table 1 Maximum Signaling Rates for Various Cable Lengths gives an estimation of maximum signaling rates versus length for most of the transceivers, CAN cables, and connectors.

Table 1 Maximum Signaling Rates for Various Cable Lengths

\begin{tabular}{|c|c|}
\hline Signal Speed (Kbps) & Bus Length (meters) \\
\hline 1000 & 30 \\
\hline 500 & 100 \\
\hline 250 & 250 \\
\hline 125 & 500 \\
\hline 62.5 & 1000 \\
\hline
\end{tabular}

ISO 11898 standard specifications recommends a maximum bus length of $40 \mathrm{~m}$, maximum stub length of $0.3 \mathrm{~m}$, and a maximum of 30 nodes.

USB to CAN converter marketed by Grid-Connect provides a way to link CAN bus to the PC. Provided with the necessary drivers and the basic CAN software, this is a ready to use device. Figure 3 shows the PCAN USB and the DB9 connector. This converter can be used to send messages to the testbed directly from a PC.

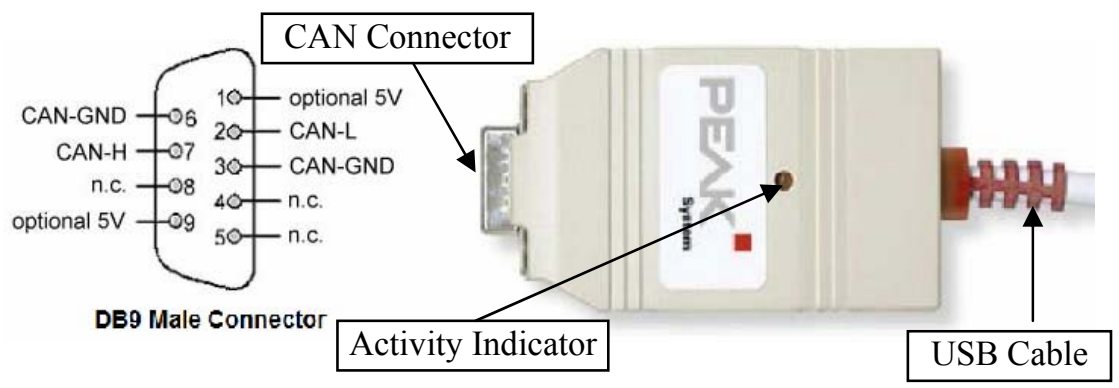

Figure 3: PCAN-USB and DB9 Connector

\subsubsection{Summary on communication}

An agent based distributed control requires that all agents are able to communicate independently with neighboring agents. Fast reaction time with the ability to transmit information without requiring a token or permission can be considered as an important criterion in an agent based environment. SPI, $\mathrm{I}^{2} \mathrm{C}$ and LIN offer master-slave communication only. GPIB is based on a parallel data interface and requires bulky interface hardware. CAN offers truly multi-master communication standard through a pair wired differential bus. 


\subsection{Development System}

At the end of the last decade, the world saw a rapid development in the embedded controllers market place. Different versions of microcontrollers and digital signal processors were developed targeting different levels of applications. Digital controllers are still shrinking in size and power consumption yet providing more processing power and features. Today resources like analog to digital conversion, pulse width modulation, interrupts, timers and communication interfaces are very well integrated within a single chip reducing time and cost of development among other benefits.

\subsubsection{Development Board}

MC9S12C32 are HC-12 core 16-bit microcontrollers with a fixed point processor that can work at up to $25 \mathrm{MHz}$ clock speed. Figure 4 summarizes resources made available by the processor [19].

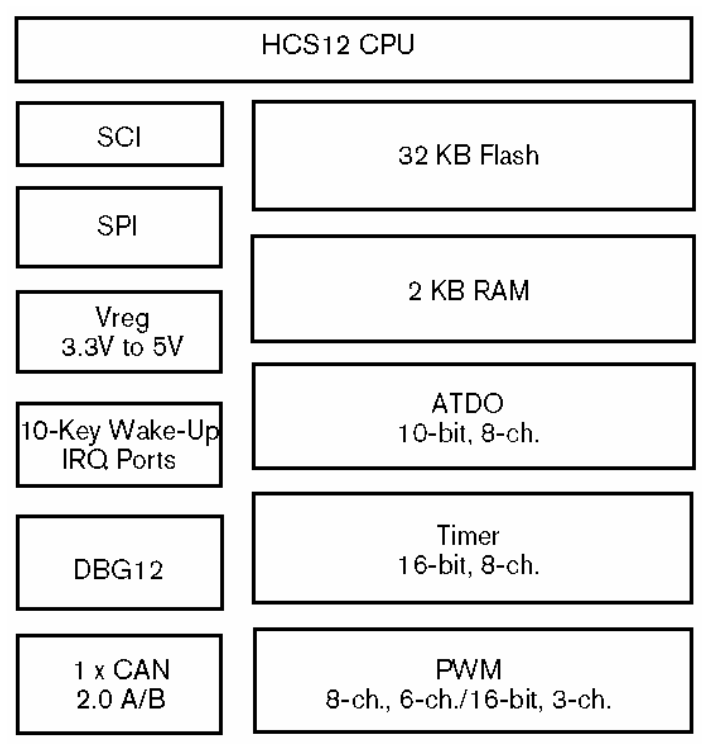

Figure 4: MC9S12C32 Microcontroller Overview.

CSM-12C32 is a MC9S12C32 based development board manufactured by Axiom Manufacturing [3]. This board offers on-board voltage regulator and a RS232 voltage level converter. It also offers two user programmable push-buttons and two user programmable LEDs. As many as thirty input and output lines are made available through a 40 pin connector. 
Figure 5 shows the $2 \frac{1}{2}$ " $\times 1 \frac{1}{2}$ " development board. A DB-9 connector is available for RS232 serial port and CAN and SPI are made available at the connectors.

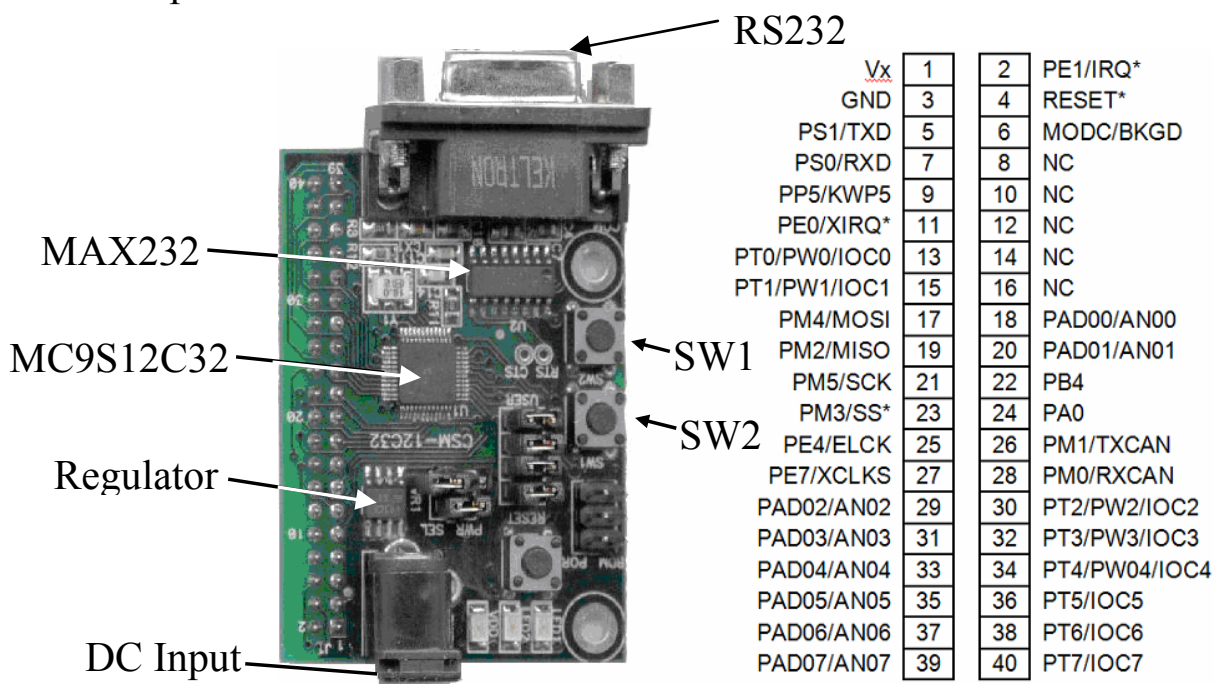

Figure 5: CSM-12C32 and Pin Outs.

\subsubsection{Integrated Development Environment}

Integrated Development Environments have been in the market for a long time now. They make the development process faster and efficient by integrating all code development needs into one package. Some of these IDEs offer features that allow developers to implement their concept even without having a detailed knowledge about the architecture of the processor itself.

Freescale makes available a version of CodeWarrior IDE to program their MC9S12C32 microcontroller. This is a fully integrated development environment, which includes simulation, in-circuit debugging and features like "Processor Expert" [20] [21] [26]. Processor expert provides a pre-written library as well as initialization codes for most of the resources available in the processor. Processor expert provides a drag-and-drop environment and the user has to write application specific portion of the code only. Mathworks has done one step further by providing a user friendly development environment. MATLAB/Simulink allows, through integrated embedded target development tools, a graphic drag-and-drop programming interface for developing realtime embedded applications [53]. 


\subsubsection{Integrated Simulink Development Environment}

Coupled with Real-Time Workshop and the embedded target development for Motorola HC12 toolbox, Simulink can automatically generate C code for real-time embedded applications. As the efficiency and flexibility of the code improves, this is becoming more widely adopted for rapid prototyping and even production systems.

Real-time Workshop uses the Target Language Compiler (TLC) to generate target specific $\mathrm{C}$ codes [30]. TLC provides an open environment for incorporating customized code to improve algorithms and code efficiency or size. A TLC file corresponding to a Simulink block includes the header and the parameter information taken from the Simulink block GUI (see Fig. 6).

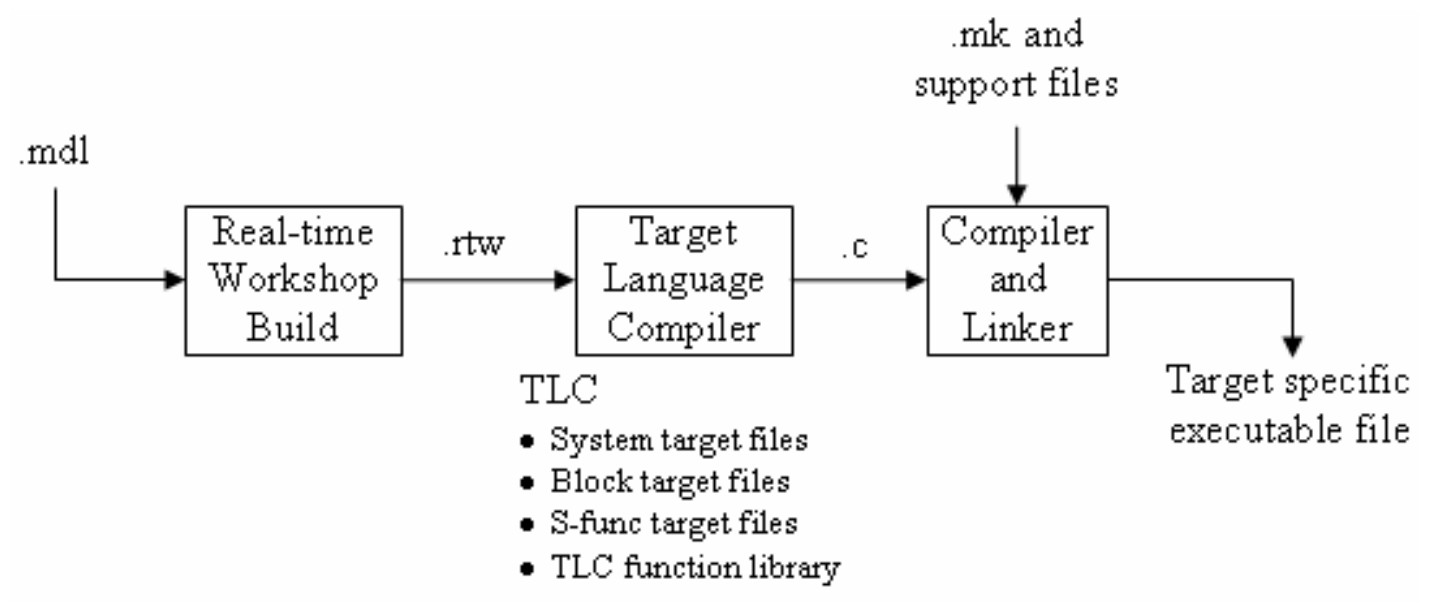

Figure 6: Code Generation Process Flowchart.

A model file with the .rtw extension contains very high-level language semantics for a Simulink block. TLC uses these .rtw files to generate a project in $\mathrm{C}$ or $\mathrm{C}++$ by using template makefiles. Template makefiles contain all the appropriate $\mathrm{C}$ or $\mathrm{C}++$, compiler, and compiler options information for the build process.

TLC uses its library of functions to transform two classes of target files: system target files are used to specify the overall structure of the generated code, while the block target files are used to implement functionality of individual Simulink blocks, including userdefined s-function blocks. Block target files can fully inline block functionality into the body of the generated code. 
TLC generates "inlined" code for most of the library blocks. Inlined code is more efficient as they use simpler direct code segments and techniques. Without TLC, Sfunction methods would be generated to initialize, run, and terminate a block making them bigger in size and slower to run. The advantage of "inlined" code can be demonstrated with the following example [54],

In this Simulink model a bitwise operator is connected to the output of an adder.

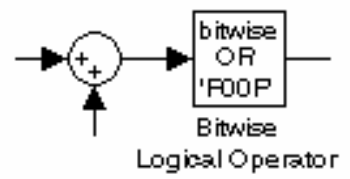

Figure 7 show the code generated using TLC. There is no initialization or setup code required for this block if the code is inlined.

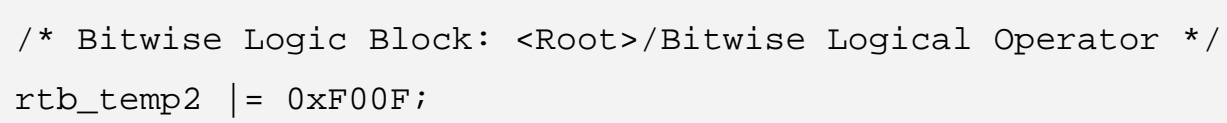

Figure 7: Inlined Code

The code generated for the bit-wise operator block reuses a temporary variable that is set up for the output of the sum block to save memory. If this block is not inlined, the source code for the S-function itself with all its various options would have been added to the generated code base as shown in Figure 8.

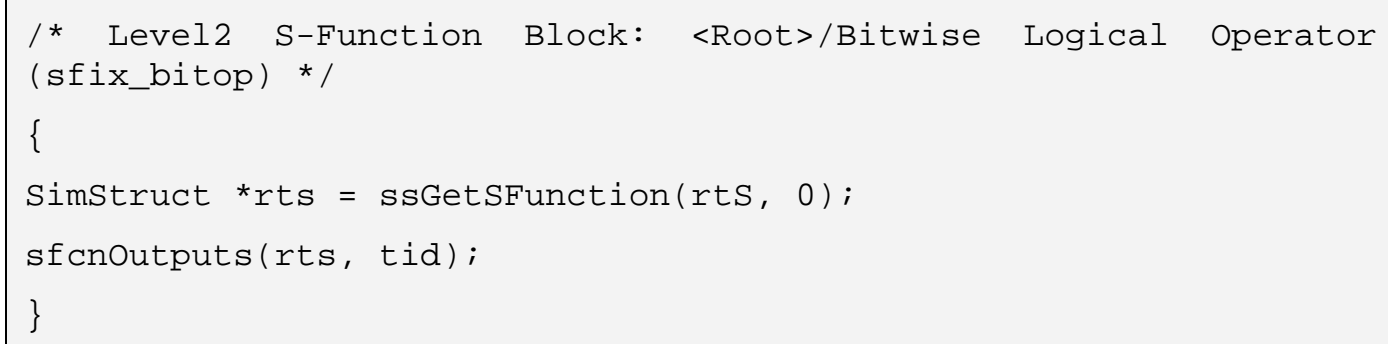

Figure 8: Noninlined Code. 


\section{Chapter 3 HARDWARE DESIGN}

This chapter addresses the converters topologies, control design, and implementation of the shipboard power system testbed.

\subsection{Introduction}

The shipboard power system is a combination of AC and DC power distribution components. The larger loads are electrically closer to the generators but use AC/DC converters for improved performance. One of the large loads is the propulsion drive, which consumes a major part of the on-board power. Pulsed power loads are short in duration but require a very high energy density and are also connected to the $\mathrm{AC}$ bus through converter technology. Other loads on the shipboard are supplied using a DC distribution system. AC-DC, DC-DC and DC-AC power converters are therefore fundamental components and form the backbone of the DC distribution system. A DCDC converter acts like a transformer in an AC system. There are several topologies available to implement DC-DC conversion and they all come with some advantages and disadvantages [35]. 


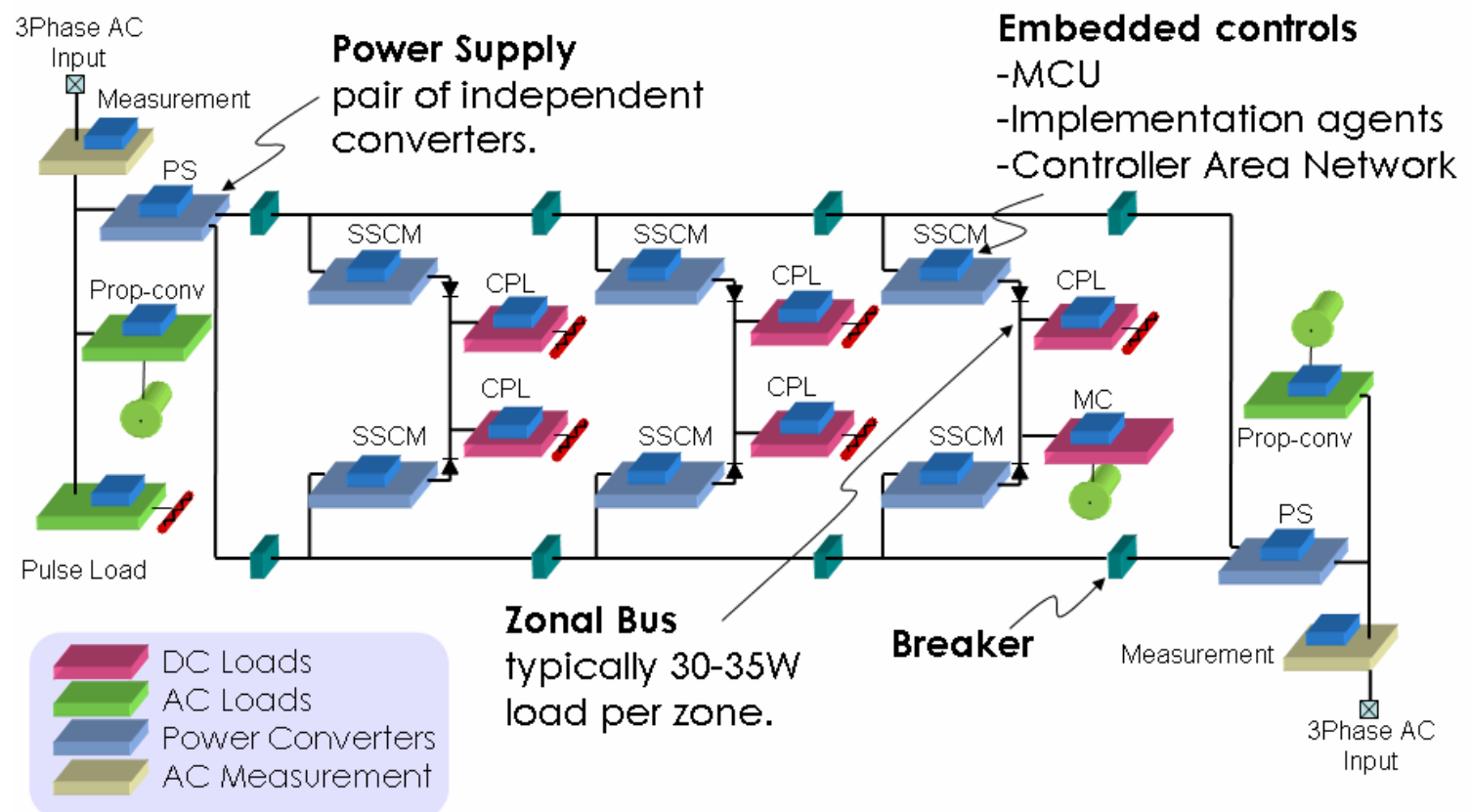

Figure 9: Testbed Components

Figure 9 shows the block diagram of the hardware prototype. The cascaded converters provide power to the main DC and Zonal buses. To make the design compact, nonisolated topology is selected for all the DC-DC conversion needs. Motor drives are built using IGBT based 3-phase bridges. Separate microcontroller units (MCU) are employed to control each of these devices. Functions that can establish communication with and implement decisions made by Reconfiguration Agents (RA) and Command and Control Center (CCC) reside in each of these MCUs on the testbed. This decision implementing portion of the hardware is called the Implementation Agent (IA) [42]. IAs are located at sensors and actuators throughout the testbed and integrated with the hardware. Reconfiguration layer agents are software components and executed on computers running the reconfiguration algorithm.

Along with the IAs, the MCUs are also programmed to be dedicated controllers. These controllers perform real-time voltage regulation and include current and power limiting functionality, depending upon the requirements of the device being controlled.

The zonal architecture and its desired reliability level require that at least two Shipboard Service Converter Modules (SSCM) provide power to the zonal bus. This arrangement provides several configuration options by providing a number of power routing paths. A 
zonal bus in Figure 9 is connected to a pair of such converters. "ORing" diodes are used at the output of these converters to block reverse current flows.

\subsection{Selecting Operating Voltages and Currents}

One of the most important criteria to consider while building a testbed for general use is safety. For an electric testbed safe voltage levels are important and need consideration. Occupational Safety and Health Administration (OSHA) sets guidelines for voltage safety limitations. OSHA guidelines consider voltages under $50 \mathrm{~V}$ safe in all conditions [40]. Voltages under $500 \mathrm{~V}$ can still be considered non lethal under dry conditions [9]. Looking at the workplace safety, electric current below $6 \mathrm{~mA}$ is not considered lethal. Under dry conditions or standing on insulated floor, typical body resistance is $100 \mathrm{k} \Omega$. A wet body or wet ground can reduce the resistance bringing body-to-ground resistance below $10 \mathrm{k} \Omega$.

Conduction losses in a buck converter occur due to the drop across the on-resistance of the MOSFET. Hence, losses are a function of the square of the load current and the output to input voltage ratio, while its dynamic losses are a function of load current, input voltage, rise, and fall time. Input voltages have been selected for most of the converters here, at least $15 \mathrm{~V}$ above the desired output voltages, to keep large enough margins for regulation and control and conduction losses. Maximum achievable line to line voltage for the motor drives can be directly calculated by using, $V_{d c} /(2 \sqrt{ } 2)$. Figure 10 shows selected voltages for different testbed buses. 


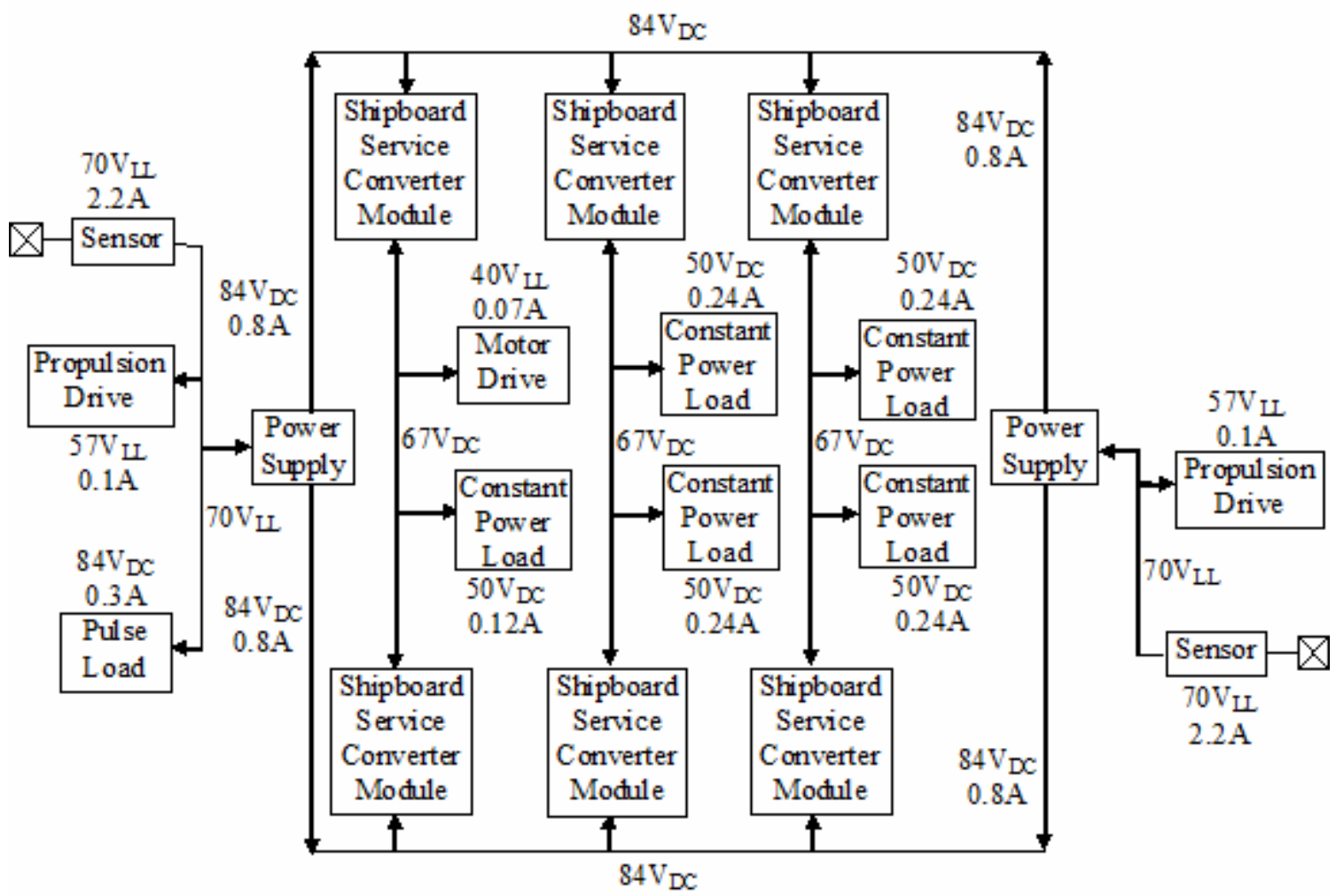

Figure 10: Voltage Assignment and Estimated Current

\subsection{Power Supply}

The Power Supply (PS) is based on 3-phase rectifier at its input and non-isolated stepdown DC-DC converter at the output. The converter comprises of a MOSFET based high side switching regulator. The output of the converter uses a passive low-pass filter built using an inductor and a capacitor. The power supply integrates two such converters and these operate from the same rectified power source. Figure 11 shows a basic line diagram of a power supply.

Optically isolated gate drive circuits must be used as the converters are non-isolated while at the same time the microcontrollers use a non-isolated communication network. The isolated drive circuit is designed to float at the potential of the MOSFET's source. A pulsed transformer can be used to drive the gate of the MOSFET; however, it requires a high frequency step-up transformer, which would burden the MCU output with a heavy load at lower frequencies. Instead, this testbed adapts an active gate drive circuit in which 
current does not vary with the switching frequency. This design gives more freedom in selecting a switching frequency without worrying about exceeding the current rating.

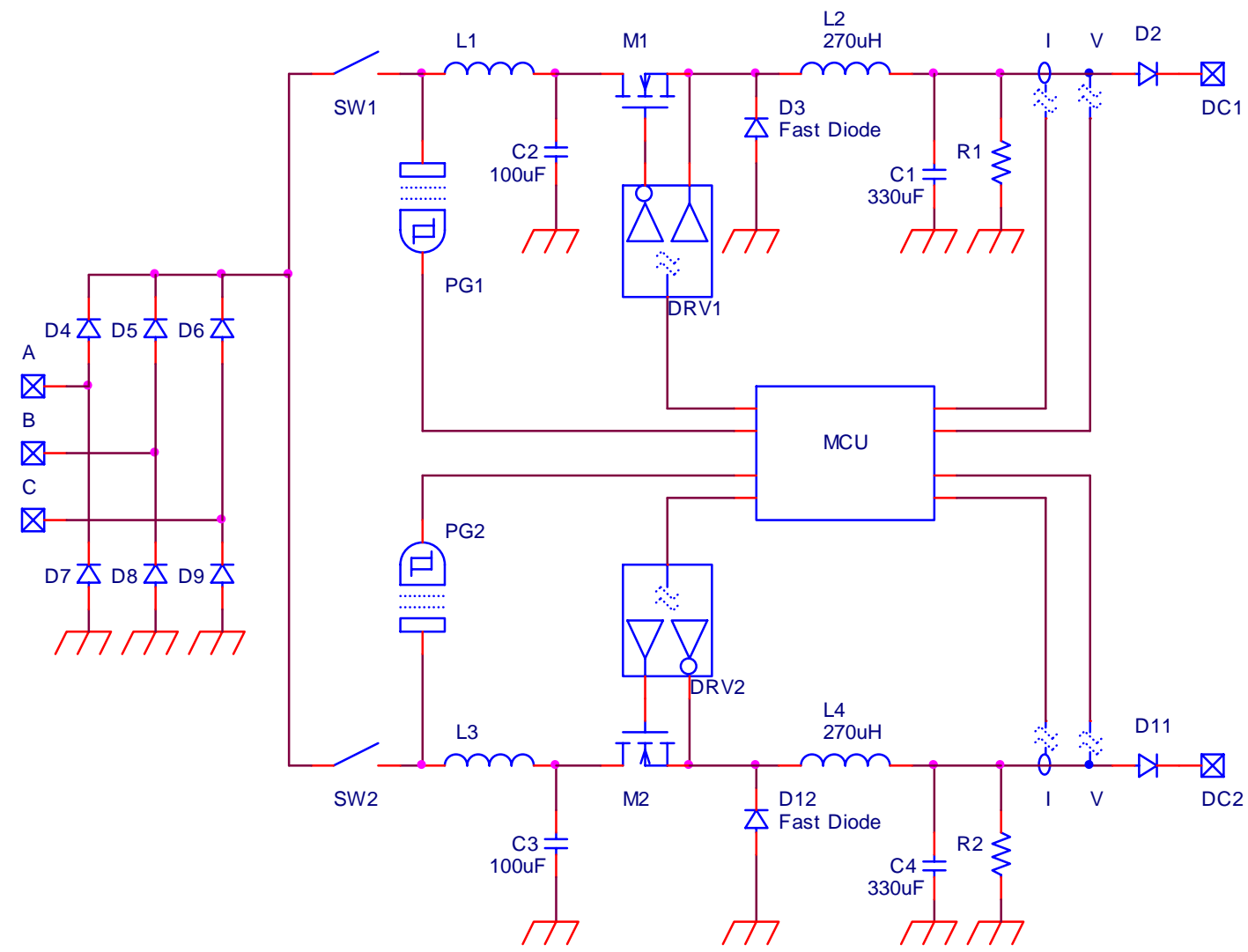

Figure 11: Power Supply Line Diagram.

\subsubsection{Selection of Switching Device}

Based on controllability, switching devices can be classified into three main groups: uncontrollable by switching signals, partially controllable by switching signals, and fully controllable by switching signals. Diodes fall under the first category because they do not have any control signal input. Thyristors fall under the second category, which can be turned on by a signal but turning off requires special arrangements. GTO, BJT, MOSFET and IGBT fall under the last category and provide full control over their switching [35][34]. The latter category can be further sub-divided into isolated and non-isolated input devices. Drive power requirements for non-isolated devices are much higher than for isolated devices like MOSFET and IGBT. MOSFET provides better frequency performance while IGBT is the better choice for high voltage applications. All of the DC-DC converters on the testbed operate at frequencies higher than $25 \mathrm{kHz}$ making 
MOSFET a better choice [37]. Motor drives, however, use IGBT as they operate at lower frequency than the converters.

\subsubsection{Selection of Switching Frequency}

An important aspect to consider while selecting switching frequency is the losses in the semiconductor devices. MOSFET switching losses are given by equation (1).

$$
P_{\text {SwLoss }}=\frac{1}{2}\left(V_{\text {in }}-V_{\text {out }}\right) \cdot I_{L} \cdot f \cdot\left(t_{c(\text { on })}+t_{c(\text { off })}\right)
$$

Considering the output voltage $\left(V_{\text {out }}\right)$, input voltage $\left(V_{\text {in }}\right)$, inductor current $\left(I_{L}\right)$, and the crossover intervals $\left(t_{o n}\right.$ and $t_{\text {off }}$ ) to be constant, the switching losses have a direct relationship with the switching frequency [35]. Typically, the modern switching MOSFETs have $t_{o n}$ and $t_{\text {off }}$ in the order of 100ns. Therefore, a switching frequency of $100 \mathrm{kHz}$ keeps the switching losses below $1 \%$ of the delivered power. Any frequency below $100 \mathrm{kHz}$ will produce less switching losses.

A higher switching frequency is desired as it reduces the size of filter elements in the circuit. However, it also increases the switching losses as already explained. Hence, a higher frequency would cause an increase in the size of the heatsink. Consequently, a balance between those two properties has to be found. Typically frequencies between 25 and $50 \mathrm{kHz}$ offer the best tradeoff between size of heatsink and size of filter elements. Going higher than $50 \mathrm{kHz}$ offers only marginal advantages [40].

The frequency is also limited by the ability of the MCU platform used to implement the controls. A desired frequency may not be achievable by the microcontroller. Considering $16 \mathrm{MHz}$ bus clock for the Freescale's MC9S12C32 microcontroller, a summary of possible PWM frequencies using A, B, SA and SB clocks is listed in Table 2 [55]. 
Table 2: Clock Speed vs PWM Frequency for MC9S12C32

\begin{tabular}{|r|r|r|}
\hline Prescaler & Clocks A, B & Clocks SA, SB \\
\hline 1 & $31.250 \mathrm{kHz}$ & $122.070 \mathrm{~Hz}$ \\
\hline 2 & $15.625 \mathrm{kHz}$ & $61.035 \mathrm{~Hz}$ \\
\hline 4 & $7.813 \mathrm{kHz}$ & $30.518 \mathrm{~Hz}$ \\
\hline 8 & $3.906 \mathrm{kHz}$ & $15.259 \mathrm{~Hz}$ \\
\hline 16 & $1.953 \mathrm{kHz}$ & $7.629 \mathrm{~Hz}$ \\
\hline 32 & $976.563 \mathrm{~Hz}$ & $3.815 \mathrm{~Hz}$ \\
\hline 64 & $488.281 \mathrm{~Hz}$ & $1.907 \mathrm{~Hz}$ \\
\hline 128 & $244.141 \mathrm{~Hz}$ & $0.954 \mathrm{~Hz}$ \\
\hline
\end{tabular}

A PWM frequency higher than $31.25 \mathrm{kHz}$ can only be achieved at the cost of loosing resolution. A $62.5 \mathrm{kHz}$ PWM will only offer 7 bit resolution for the PWM and it keeps on decreasing until $8 \mathrm{MHz}$ when the PWM will be left with 1 bit resolution. $31.25 \mathrm{kHz}$ is the best frequency for the testbed considering all the aspects stated above.

\subsubsection{Selection of Inductor}

Buck converter can be operated in the continuous or discontinuous mode. Control in the continuous current mode, or CCM, is more stable as it does not require capacitor current during steady state operation. When the converter is lightly loaded causing the inductor current to decrease, the converter could run into discontinuous mode. Inductor can be selected to set the boundary between continuous and discontinuous mode. Here the inductor can be chosen to operate in CCM. Considering this as the criteria, inductor value can be selected by using equation (2).

$$
L=\frac{\left(V_{i n N}-V_{o u t}\right) \cdot V_{o u t}}{2 \cdot V_{i n N} \cdot I_{L N} \cdot f_{S}}
$$

Considering a nominal input voltage of $97 \mathrm{~V}$ and an output voltage of $84 \mathrm{~V}$, a load current of $0.9 \mathrm{~A}$, switching frequency of $31.25 \mathrm{kHz}$, the required value is $200 \mathrm{uH}$. The higher the inductor value the more it guarantees CCM operation. Inductor currently used in the converters is $270 \mathrm{uH}$ with $\pm 20 \%$ tolerance, a DC resistance of $0.75 \Omega$ and a maximum current carrying capacity of $0.90 \mathrm{~A}$. 


\subsubsection{Selection of Capacitor}

The output voltage ripple is defined by the capacitor value and its selection is important to minimize ripple voltage. The two important capacitor parameters to be considered are the capacitance and the equivalent series resistance (ESR). The third parameter the equivalent series inductance (ESL), does not become significant unless the frequency is in the order of $100 \mathrm{kHz}$. ESR helps with respect to controller stability by providing damping at higher frequencies. ESR in large capacitors is significant for frequencies as low as $10 \mathrm{kHz}$. ESR is inversely proportional to capacitance. For aluminum electrolytic capacitors it can be roughly estimated by dividing $5 \times 10^{-5}$ by the value of capacitance in farads [46].

The ripple voltage is the drop across ESR, which can be determined by multiplying ESR with the ripple current. Ripple voltage can be brought down by choosing capacitors with low ESR. Rise in current within $50 \%$ duty cycle ( $1 / 2 T$ time) can be estimated, using the following equation.

$$
\Delta I=\frac{\left(V_{\text {inN }}-V_{\text {out }}\right) \cdot T}{2 L}
$$

Using known values of $V_{i n N}, V_{\text {out }}, T$, and $L, \Delta I$ is calculated to be $0.83 \mathrm{~A}$. In order to bring the ripple voltage below $4 \mathrm{~V}(5 \%)$, a capacitor with an ESR below $1 \Omega$ is required. The following relationship between C and ESR can be used to estimate the capacitance [36]

$$
C=\frac{\tan \delta}{\omega \cdot E S R}
$$

where $\tan \delta$ is the dissipation factor and $\omega$ is the frequency. The aluminum capacitor used is $330 \mathrm{uF}$ at $250 \mathrm{~V}$ with an ESR of $0.8 \Omega$ at $120 \mathrm{~Hz}$ [39].

\subsubsection{Selection of Voltage and Current Sensors}

Accuracy of sensors is important for the performance of components and the overall system. Non isolated sensing techniques including voltage dividers and current shunts are not suitable for cascaded or largely integrated systems, while isolated ones may be bulky and expensive or may degrade linearity and add offset. As the controllers must share a 
global ground due to the non-isolated nature of the CAN communication link, isolation is required between the power electronics and the controllers.

Isolation can be achieved in several ways. With an isolation amplifier some significant non-linearity and offset can be expected and need to be compensated. With voltage source sensors, calibration may be required for the voltage losses incurred during signal transmission. Linear opto-isolators are available but requirement to meet the diode forward voltage makes them an unsuitable choice for low signal applications. A better way is the use of isolated sensors.

Hall-effect sensors with current source output are available with a precision of up to $\pm 0.5 \%$ and linearity of $\pm 0.2 \%$. Use of such sensors reduces the number of external components and allows for a more compact design and also reduces possible points of failures.

An advantage of having a current output sensor is the omission of signal losses due to cable resistance. Signal can be transmitted or rectified without losses and current signals also offer better noise immunity. LEM produces small hall-effect sensors with a typical isolation voltage of $1.7 \mathrm{kV}$. Both, current and voltage sensors are based on current transformation. These sensors have fixed output to input ratios known as conversion ratio [27]. A $10 \mathrm{~mA}$ sensor in combination with proper current limiting resistor acts like a voltage sensor.

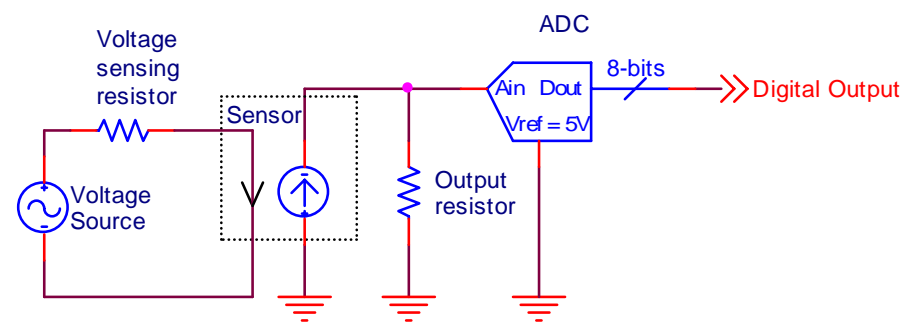

Figure 12: Hall Effect Voltage Sensor.

Figure 12 shows the voltage sensor and the ADC connected to a voltage source through a current limiting resistor. The LV 25-NP datasheet recommends that a $200 \Omega$ resistor be used between the output and ground for the sensing [28]. If the ADC output value ( $\mathrm{Val})$ is known, measured voltage can be determined by using equation (5). 


$$
V_{\text {mea }}=\frac{R_{S} \cdot V_{A D C-r e f}}{C R \cdot R_{M} \cdot 2^{A D C-b i t}} \cdot V a l
$$

Where, the reference voltage of the ADC $\left(V_{A D C-r e f}\right)$ is $5 \mathrm{~V}$, conversion ratio of the halleffect sensor $(C R)$ is 2.5 , measurement resistor $\left(R_{M}\right)$ is 200 , and the number of ADC bits (ADC-bit) is 8. Using these values the equation (5) can be simplified to equation (8).

$$
V_{\text {in }}=\frac{R}{25500} \cdot \mathrm{Val}
$$

Value of the resistor $(R)$ can be chosen to suit the voltage range to be measured.

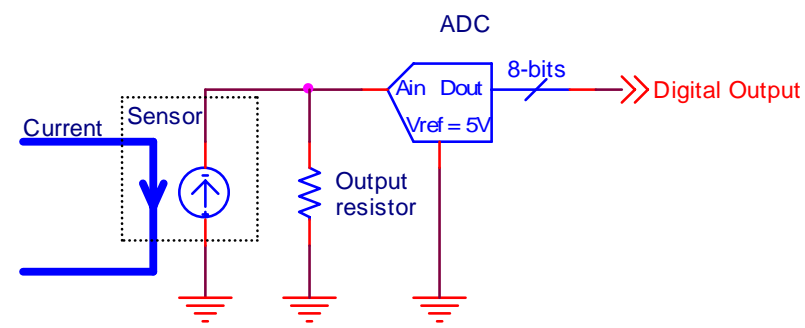

Figure 13: Hall Effect Current Sensor.

Figure 13 shows the current sensor and ADC connected to a current carrying conductor. The LA 25-NP datasheet also recommends a $200 \Omega$ as the output resistor [28]. If the $\mathrm{ADC}$ output value $(\mathrm{Val})$ is known, measured current can be determined by using the equation (7).

$$
I_{\text {mea }}=\frac{V_{A D C-r e f} \cdot C R}{R_{M} \cdot 2^{A D C-b i t}} \cdot V a l
$$

Where, the reference voltage of the $\mathrm{ADC}\left(V_{A D C \text {-ref }}\right)$ is $5 \mathrm{~V}$, measurement resistor $\left(R_{M}\right)$ is 200 , and the number of ADC bits (ADC-bit) is 8 . The conversion ratio of the hall-effect sensor $(C R)$ varies with the selection of sensors. Using these values equation (7) can be simplified to equation (8).

$$
I_{\text {in }}=\frac{C R}{10200} \cdot \mathrm{Val}
$$




\subsubsection{Optical Isolation and Drive Circuit}

A MOSFET, being an insulated gate device, has a very high input impedance. Most of the MOSFET datasheets specify nano-amps of DC leakage current between gate and source. However, the parasitic capacitance between the gate and the channel as well as along the channel makes the $\mathrm{AC}$ drive current higher at higher frequencies. An $\mathrm{AC}$ applied at the gate sees $\mathrm{C}_{\text {iss, }}$, the input capacitance across gate to source and $\mathrm{C}_{\mathrm{rss}}$, the reverse transfer capacitance across gate and drain [46].
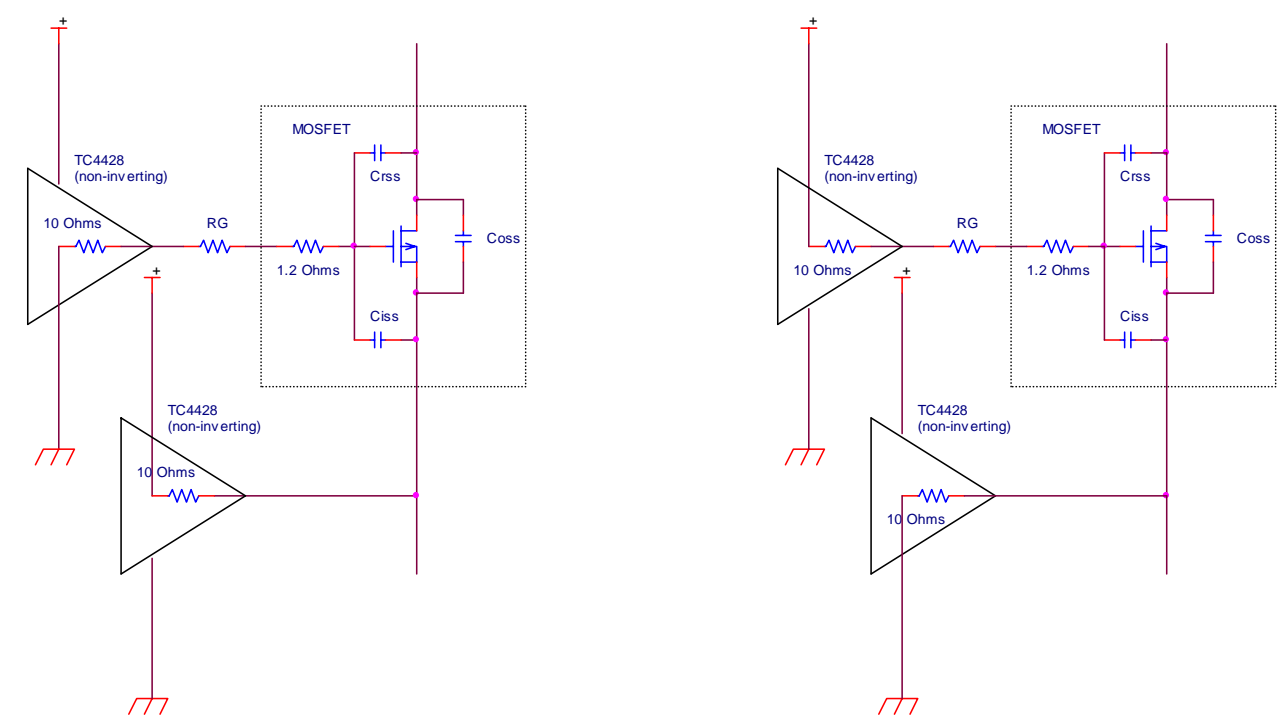

Figure 14: MOSFET Gate Driver Turned-On and Turned-Off Mode of Operation.

A gate resistance is required in order to limit gate current. A high gate current, however, increases switching losses. Another aspect to consider while driving a MOSFET is that the drive must meet the drive voltage requirement of the MOSFET, typically $10 \mathrm{~V}$.

An opto-isolator can be used to isolate controller from the gate drive circuit. An optoisolator with Schmitt trigger output will be required to further reduce switching losses by providing sharp switching signals to the gate. HCPL 2211, manufactured by Hewlett Packard, offers good switching performance at all voltage levels by using hysteresis and a good slew rate performance [23]. This opto-isolator is specially designed for use with gate drives and it offers a Schmitt trigger NAND gate at the output. It can be operated from a power supply of up to $20 \mathrm{~V}$. The datasheet specifies the LED turn-on current is 0.8 $\mathrm{mA}$ and turn-off current of $0.7 \mathrm{~mA}$. The opto-isolator LED is connected to the output of the MCU, which is capable of sourcing or sinking up to $25 \mathrm{~mA}$ current [32] on its IO 
lines. To obtain the LED forward current of $1 \mathrm{~mA} @ \sim 1.43 \mathrm{~V}$, a resistor of $3.57 \mathrm{k} \Omega$ or smaller should be used.

Gate drive is one of the most important parts of the design as it contributes to the switching performance, which is reflected in the switching losses of the converter. The optimal value of gate resistor is the one that gives maximum current without exceeding absolute maximum rating of the gate drive. Gate to source and gate to drain capacitance play important roles while sizing the gate drive circuit [3].

Figure 14 shows the connections within the drives during turn on and turn off modes. A turn off applies a positive voltage at the gate with respect to the source. During a turn off, a negative voltage is applied at the gate with respect to the source, allowing a faster discharge of the $\mathrm{C}_{\text {iss }}$ capacitor and hence providing a rapid turn off reducing switching losses.

The designed gate drive uses \pm 15 volts, which is half of the gate-to-source breakdown voltage. Highest amount of current occurs while discharging the reverse capacitance, $\mathrm{C}_{\mathrm{rss}}$. At this time, $\mathrm{C}_{\text {iss }}$ will have been charged at $V_{\text {miller }}$.

Gate resistance for this discharge can be expressed by the following equation

$$
R_{G}=\frac{\left(V_{d r v}-V_{\text {miller }}\right)}{I_{\max }}-R_{d r v}
$$

Drive voltage $V_{d r v}$ is $30 \mathrm{~V}$ and the Miller voltage, $V_{\text {miller }}$, as obtained from the datasheet, is $6 \mathrm{~V}$. Maximum rated current for the driver is $1.5 \mathrm{~A}$. The driver's output impedance, $R_{d r v}$, has been specified in the datasheet as $7 \Omega$, hence the smallest value of $R_{G}$ without exceeding the maximum current rating of the driver is $9 \Omega$.

\subsubsection{Power Good Signal}

A power good signal is added to let the converter verify whether it is receiving acceptable amount of voltage from the preceding converter. The converter is disabled unless an acceptable voltage level is seen at the input. An optically isolated NAND gate featuring hysteresis is used to generate a logic low voltage when the bus voltage reaches the acceptable level. A $0.8 \mathrm{~mA}$ current is required to turn on the LED in the H11L3M opto- 
isolator, which can be produced by a voltage divider arrangement [15]. Potentiometer is used in the design to make the system testable at different voltage levels.

\subsubsection{CAN Interface and Plug-In Board Schematic}

CSM-12C32 module from Axiom Manufacturing contains an RS-232 level converter but does not include CAN driver. Hence a separate board is built with a 40 pin connector to plug-in the CSM-12C32 module. This board includes a $15 \mathrm{~V}$ to $5 \mathrm{~V}$ regulator along with filter capacitors and the SN65LBC031 chip as a non-isolated differential CAN bus driver

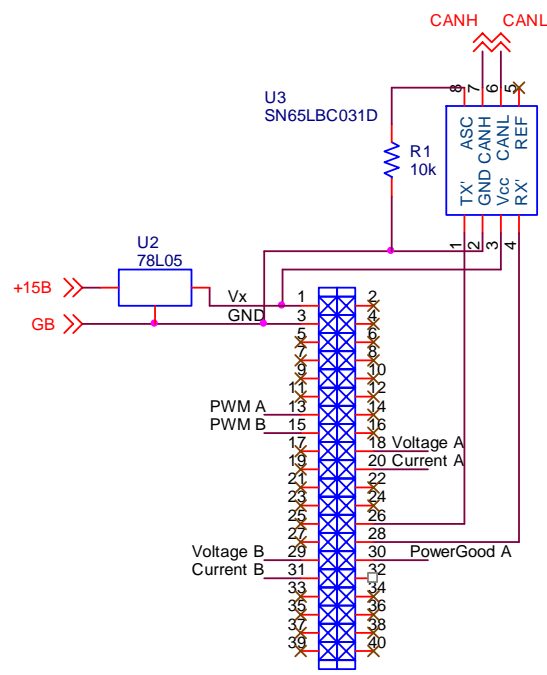

Figure 15: Schematic of MCU Plug-in Board and CAN Driver for Power Supply The main converter board contains filters for feedback signals. Since the switching frequency of $31.25 \mathrm{kHz}$ is high compared to the sampling frequency of $100 \mathrm{~Hz}$, a $20 \mathrm{~dB}$ per decade RC filter has been used.

Table 3: Table of Pin Assignment for Power Supply MCU Board.

\begin{tabular}{|l|l|l|}
\hline Pin name & Pin number & Allocated for \\
\hline PT0/PW0/IOC0 & 13 & Pulse Width Modulation A \\
\hline PT1/PW1/IOC1 & 15 & Pulse Width Modulation B \\
\hline PAD00/AN00 & 18 & Voltage Sense A \\
\hline PAD01/AN01 & 20 & Current Sense A \\
\hline PM1/TXCAN & 26 & CAN Xm't \\
\hline PM0/RXCAN & 28 & CAN Rc'v \\
\hline PAD02/AN02 & 29 & Voltage Sense B \\
\hline PT2/PW2/IOC2 & 30 & Power Good Signal for A \\
\hline PAD03/AN03 & 31 & Current Sense B \\
\hline PT3/PW3/IOC3 & 32 & Power Good Signal for B \\
\hline
\end{tabular}




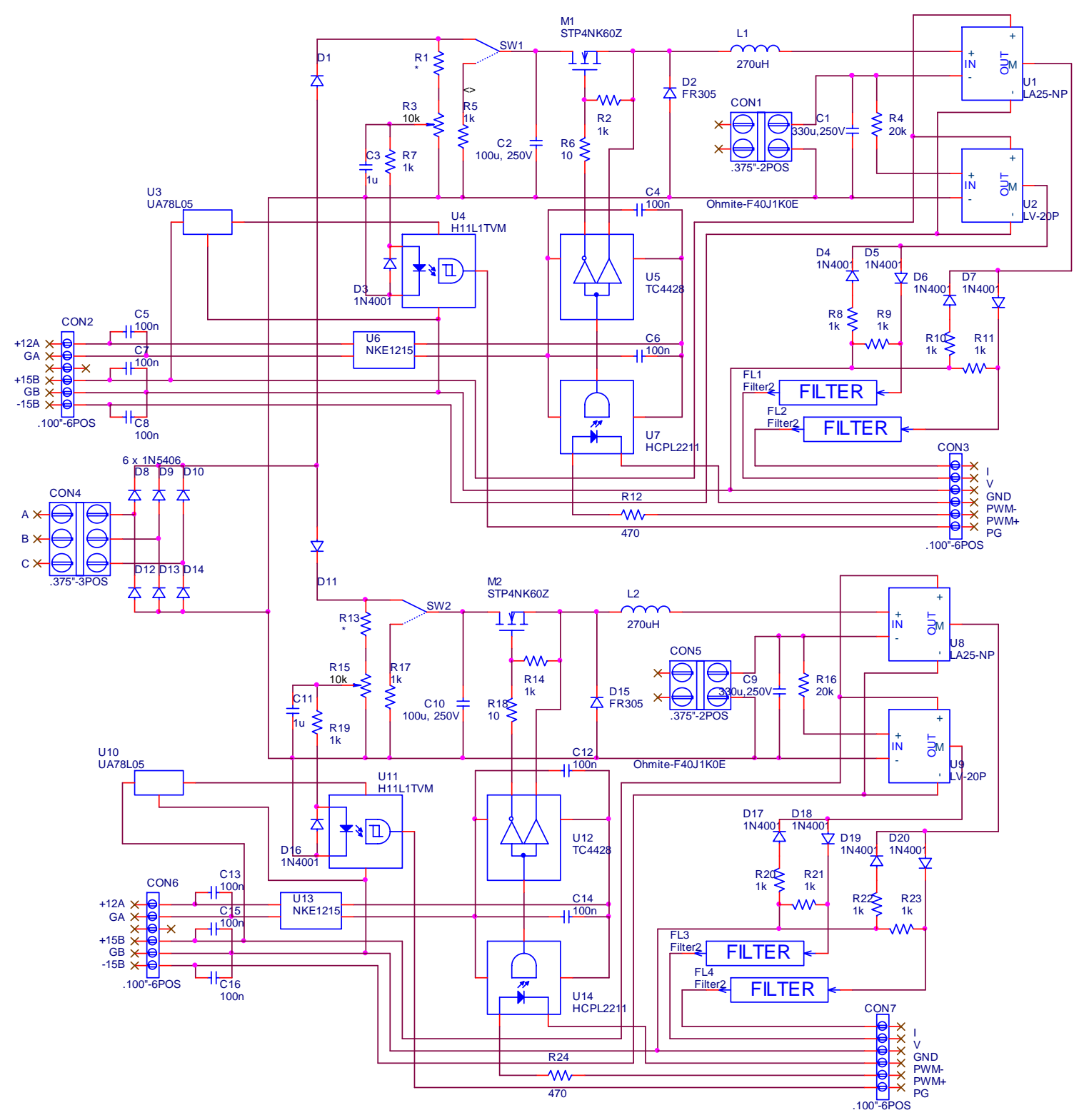

Figure 16: Detailed Schematic of Power Supply.

Two separate converters are connected to a single rectifier block and a single MCU controls them. Table 3 summarizes the allocation of MCU pins.

\subsubsection{Controller}

The sampling time plays a very important role in the stability of digitally controlled systems. The sampling frequency must be at least twice the highest frequency contained in the spectrum of the sampled signal. A faster operating digital controller allows a better 
response time. A high frequency sampling, compared to the frequency of the signal to be sampled, requires a higher resolution ADC to keep the advantages. Otherwise it only adds to the computational overhead.

Closed-loop controllers are error based feedback controllers. Here the desired value $\mathrm{r}(\mathrm{t})$ is compared in a recursive loop with the actual value $\mathrm{y}(\mathrm{t})$ and the difference is termed error. Based on this error $\mathrm{e}(\mathrm{t})=\mathrm{r}(\mathrm{t})-\mathrm{y}(\mathrm{t})$, controller generates control action $\mathrm{u}(\mathrm{t})$, which minimizes the error. Proportional-Integral controllers are most frequently used for controls. The following two points should be considered: the proportional gain amplifies the error signal and helps reduce steady state error but a high gain can make the system unstable, the integral gain integrates the error signal and holds the value. It is the only part which can carry an output for a zero input. Therefore only an integral component can eliminate steady-state errors completely. Yet, an integral action may slow down the system as old values need to be relieved first. Also an integral action increases the order of the system and can cause instability.

The linear transfer function of a PI controller is given by the equation (10).

$$
\frac{U(s)}{E(s)}=K_{P}+\frac{K_{I}}{s}
$$

Where, $K_{P}$ is the proportional gain and $K_{I}$ is the integral gain.

Figure 17 shows the Simulink model for the power supply controller. Two independent power supplies are controlled using a single MCU. For each power supply, the inner current control uses the output signal of the outer voltage loop as reference input. Current is varied to control the output voltage level. When the voltage controller's output reaches the upper saturation level, also shown in Figure 17, the controller changes to current control mode. During this mode, voltage is allowed to drop below the set-point in order to maintain current at the maximum limit. 


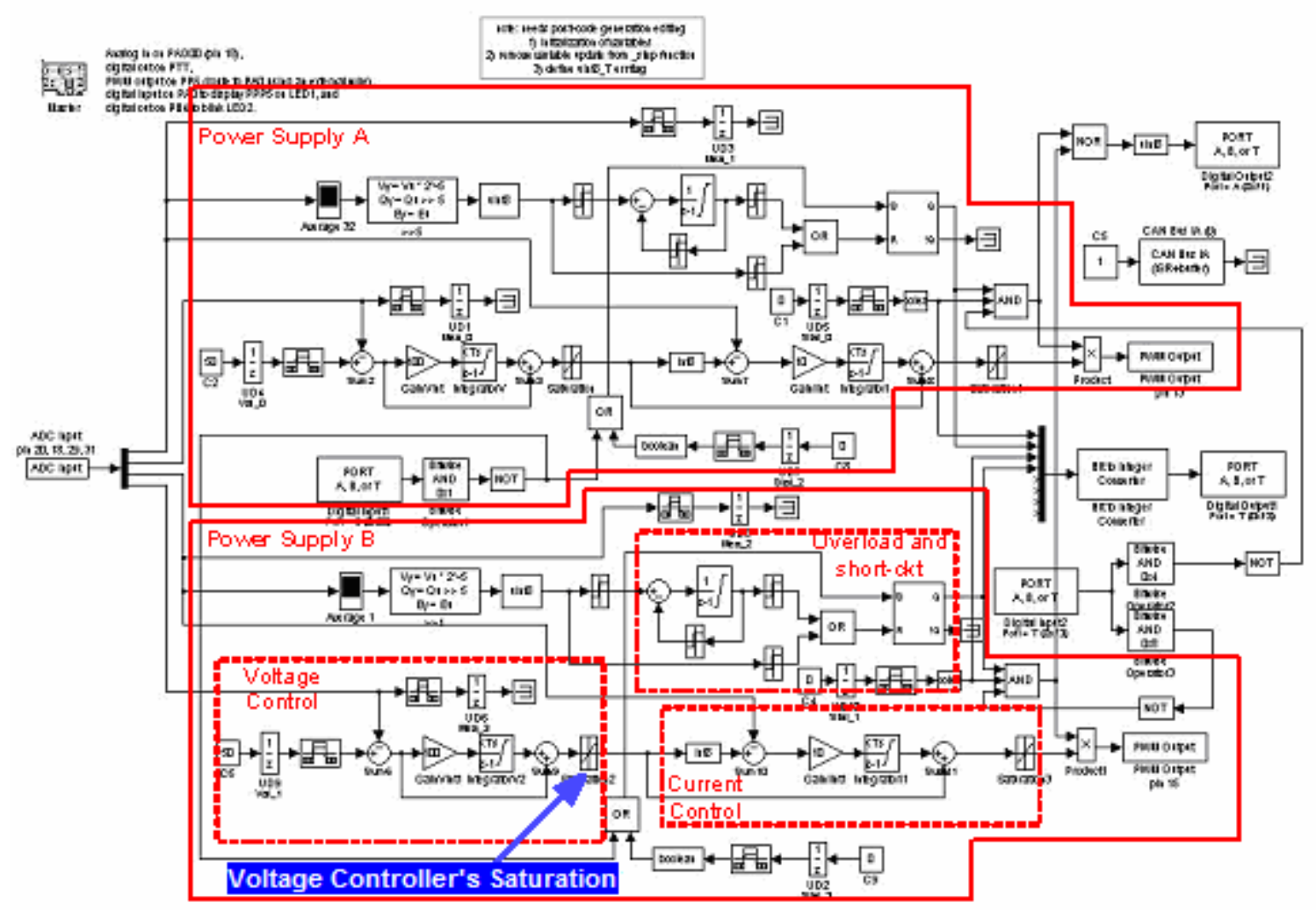

Figure 17: Simulink Model for Power Supply Controller.

This limit is set just above the overload cutoff to ensure that the power supply does not operate in current limiting mode for too long. The short circuit current level is set high enough to ensure the controller does not trigger false short circuit cutoffs. The following table summarizes the parameters available for the controllers. It also lists whether the parameter is modifiable or readable only through CAN interface.

Table 4: Power Supply Controller Parameters.

\begin{tabular}{|l|r|}
\hline Available Parameter Name & \multicolumn{1}{l|}{ Values } \\
\hline Readable and Modifiable: & $0-1$ \\
\hline On-off mode flag for Power Supply A & $0-1$ \\
\hline On-off mode flag for Power Supply B & $0-255$ \\
\hline Voltage set-point for Power Supply A & $0-255$ \\
\hline Voltage set-point for Power Supply B & $0-1$ \\
\hline Overload/short circuit reset flag for Power Supply A & $0-1$ \\
\hline Overload/short circuit reset flag for Power Supply B & $0-255$ \\
\hline Readable only: & $0-255$ \\
\hline Output voltage of Power Supply A & $0-255$ \\
\hline Load current of Power Supply A & \\
\hline Output voltage of Power Supply B &
\end{tabular}




\begin{tabular}{|l|r|}
\hline Load current of Power Supply B & $0-255$ \\
\hline Internal: & 1 \\
\hline Proportional gain of the PI voltage control for Power Supply A & 100 \\
\hline Integral gain of the PI voltage control for Power Supply A & 10 \\
\hline Integral gain of the PI current control for Power Supply A & 80 \\
\hline Current set-point for Power Supply A & 255 \\
\hline Controller saturation upper limit for Power Supply A & 0 \\
\hline Controller saturation lower limit for Power Supply A & 70 \\
\hline Overload cutoff limit for Power Supply A & 200 \\
\hline Overload cutoff delay for Power Supply A & 100 \\
\hline Short circuit cutoff limit for Power Supply A & 8 \\
\hline Voltage scaling factor for Power Supply A & 8 \\
\hline Current scaling factor for Power Supply A & $0-1$ \\
\hline Power good status for Power Supply A & 1 \\
\hline Proportional gain of the voltage control PI for Power Supply B & 100 \\
\hline Integral gain of the voltage control PI for Power Supply B & 10 \\
\hline Integral gain of the current control PI for Power Supply B & 80 \\
\hline Current set-point for Power Supply B & 255 \\
\hline Controller saturation upper limit for Power Supply B & 0 \\
\hline Controller saturation lower limit for Power Supply B & 70 \\
\hline Overload cutoff limit for Power Supply B & 200 \\
\hline Overload cutoff delay for Power Supply B & 100 \\
\hline Short circuit cutoff limit for Power Supply B & 8 \\
\hline Voltage scaling factor for Power Supply B & 8 \\
\hline Current scaling factor for Power Supply B & $0-1$ \\
\hline Power good status for Power Supply B & \\
\hline
\end{tabular}

\subsection{Motor Drive}

This drive is intended at running a 3-phase induction motor from a DC source. It is based on a 3-phase IGBT bridge converter topology, and controller provides variable frequency 3-phase PWM signals to the IGBTs.

The chosen design offers optical isolation between the control input and the power electronics. An additional programmable device is added to implement gate drive logic in order to safely operate complementary IGBTs without cross conduction. 


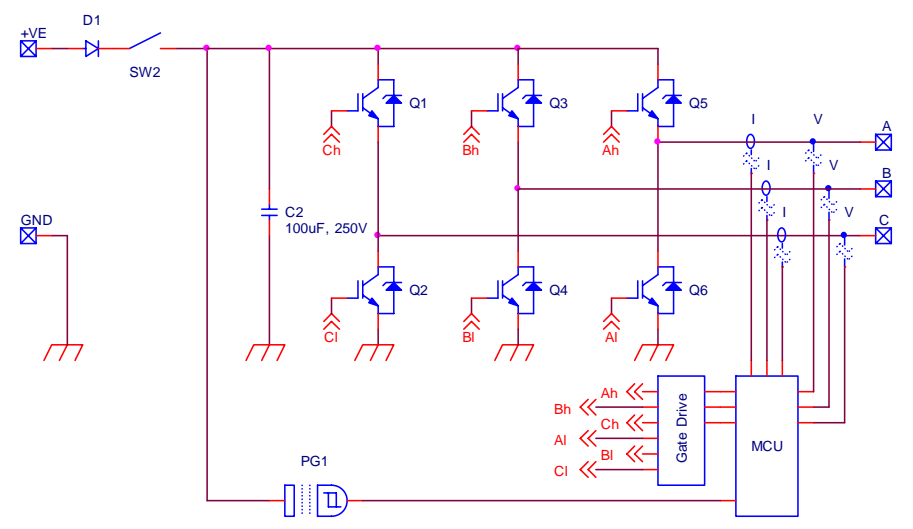

Figure 18: Line Diagram of Motor Drive.

Bootstrap diodes are used to drive high and low side IGBTs from the same power source. Current, voltage and speed sensors are provided for future developments including elaborative motor control schemes.

\subsubsection{Power Inverter Section}

The power section of the motor drive contains a 3-phase bridge inverter, which is connected to the DC voltage. The power section uses a $600 \mathrm{~V}, 6 \mathrm{~A}$ IGBT power module IRAMS06UP60A supplied by International Rectifier. This module offers a built-in gate protection logic, which disables both high and low side IGBTs if both the inputs are simultaneously enabled [24]. In this case the output voltage would be determined by the direction of current flow in the reactive load.

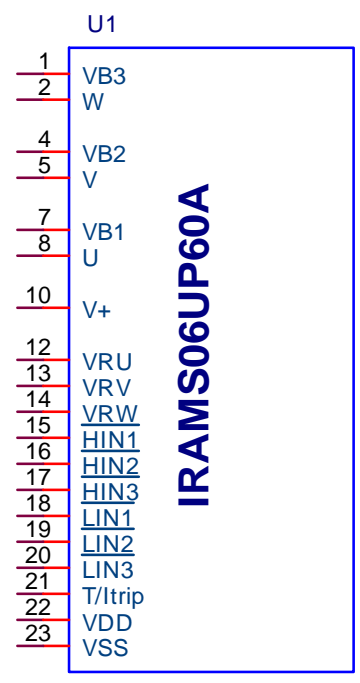

Figure 19: Integrated Power Module. 
In addition to that, the design offers a programmable GAL chip so that additional gate protection can be implemented or improved in the future. Generic Array Logic (GAL) is a programmable device in which maxterm-minterm based Boolean equations can be programmed.

This power module also offers "Itrip" which can be used if a direct over temperature shutdown is desired. A high on the input turns off the module by turning off all the IGBTs, leaving all 3-phase wires at floating state.

\subsubsection{Electrical Isolation and Gate Drive}

The controller provides PWM signals for the motor driver through optical isolators using HCPL2211. Just like mentioned in section 3.3.6, this opto-isolator offers Schmitt trigger with a better slew rate performance, and up to $1.4 \mathrm{kV}$ isolation.

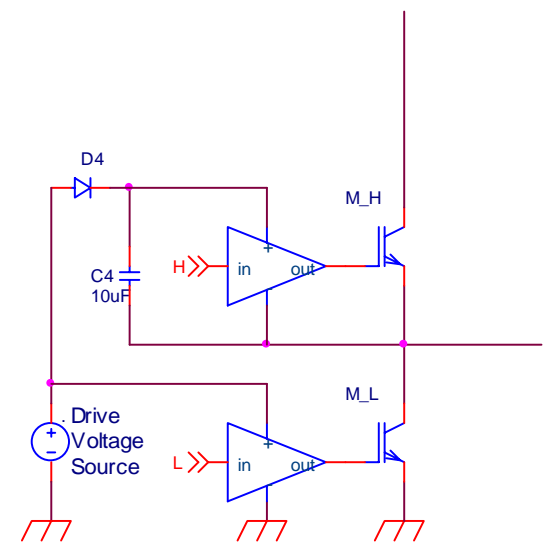

Figure 20: Bootstrap Diode Arranged for High Side MOSFET Driving.

The source pins of high and low IGBTs float at different potentials, making it essential to have isolated drive voltages for high and low side switches. Bootstrap diodes are used to charge a floating capacitor for the high side IGBT. The C4 capacitor charges through D4 diode, when M_L IGBT turns on. The stored voltage floats with respect to the power ground when M_L turns off. The capacitor voltage is always tied to the source of the high side IGBT providing the gate drive power. The programmable GAL chip provides 6 PWM outputs for the 6 different IGBTs.

Cross-conduction can occur when both switches, coupled in series, are simultaneously turned-on. Dead time ensures that both switches fully turn-off during the transition first. 
The Integrated Power Module (IPM) IRAMS06UP60A offers a built in gate protection logic. The datasheets for the IPM specify a dead-time of $290 \mathrm{~ns}$ [24].
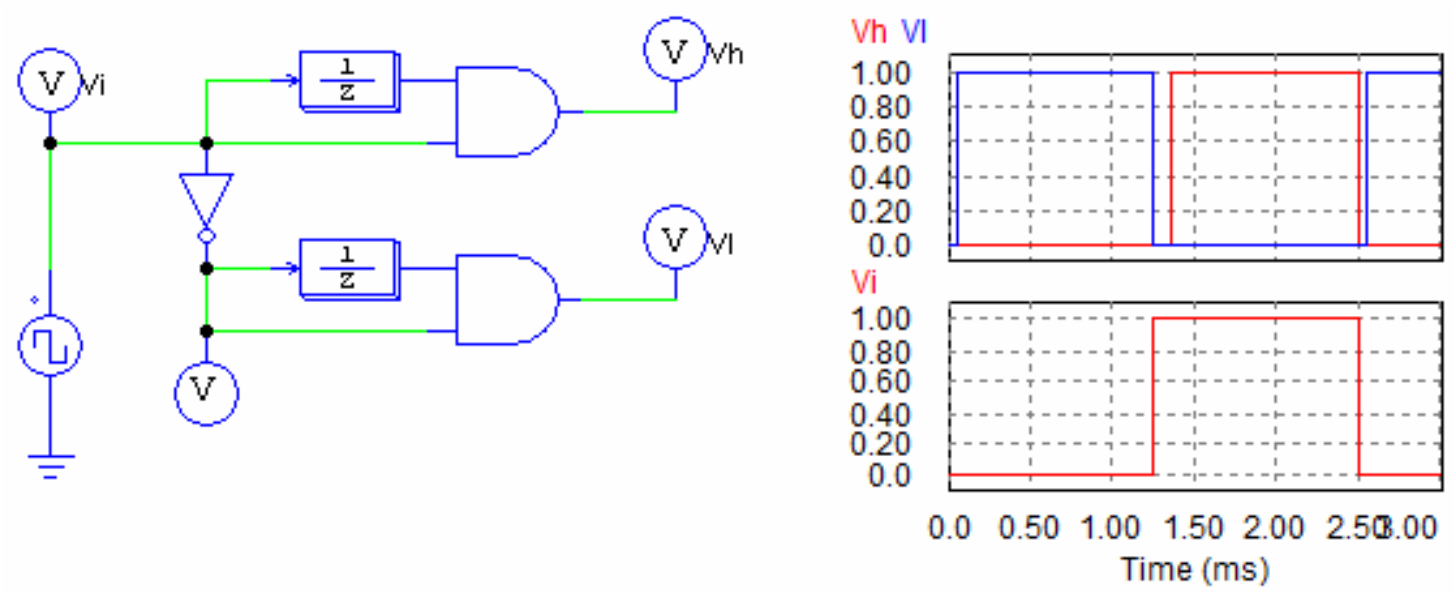

Figure 21: Dead Time Generator for Complementary IGBT

Additional dead time can be generated by using the circuit shown in Figure 21. Low side gate signal $(V l)$ turns off immediately after the input signal $(V i)$ goes high but the high side gate $(V h)$ takes about 150 ns to turn on. The delay can be generated by cascading logic gates or an RC circuit if an additional delay is required.

\subsubsection{Power Good Signal}

Motor drive monitors input DC bus voltage and provides a low signal to the controller when the bus voltage level is high enough for motor driving purpose.

\subsubsection{Schematics}

The motor drive uses three PWM outputs of the MCU to generate three phase signals. The programmable Generic Array Logic (GAL) chip generates PWM signals for the complementary IGBTs. Construction of the controller board has already been described in section 3.3.8. An analog input of the CSM-12C32 module is used as the speed set point command.

PWM output of the module drives the 25W, 115V 3-phase induction motor. Capacitors $\mathrm{C} 1-\mathrm{C} 3$, shown in Figure 23, provide drive voltage to the high side IGBTs using bootstrap diodes. Resistors R3 - R8 provide pull-up for outputs of the GAL chip. 


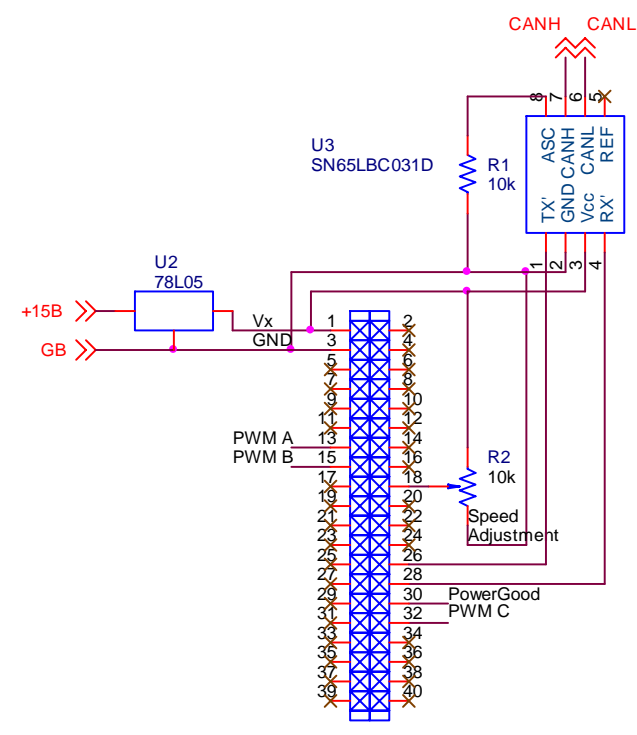

Figure 22: Detailed Schematic of MC9S12C32 Plug-in Board for Motor Drive.

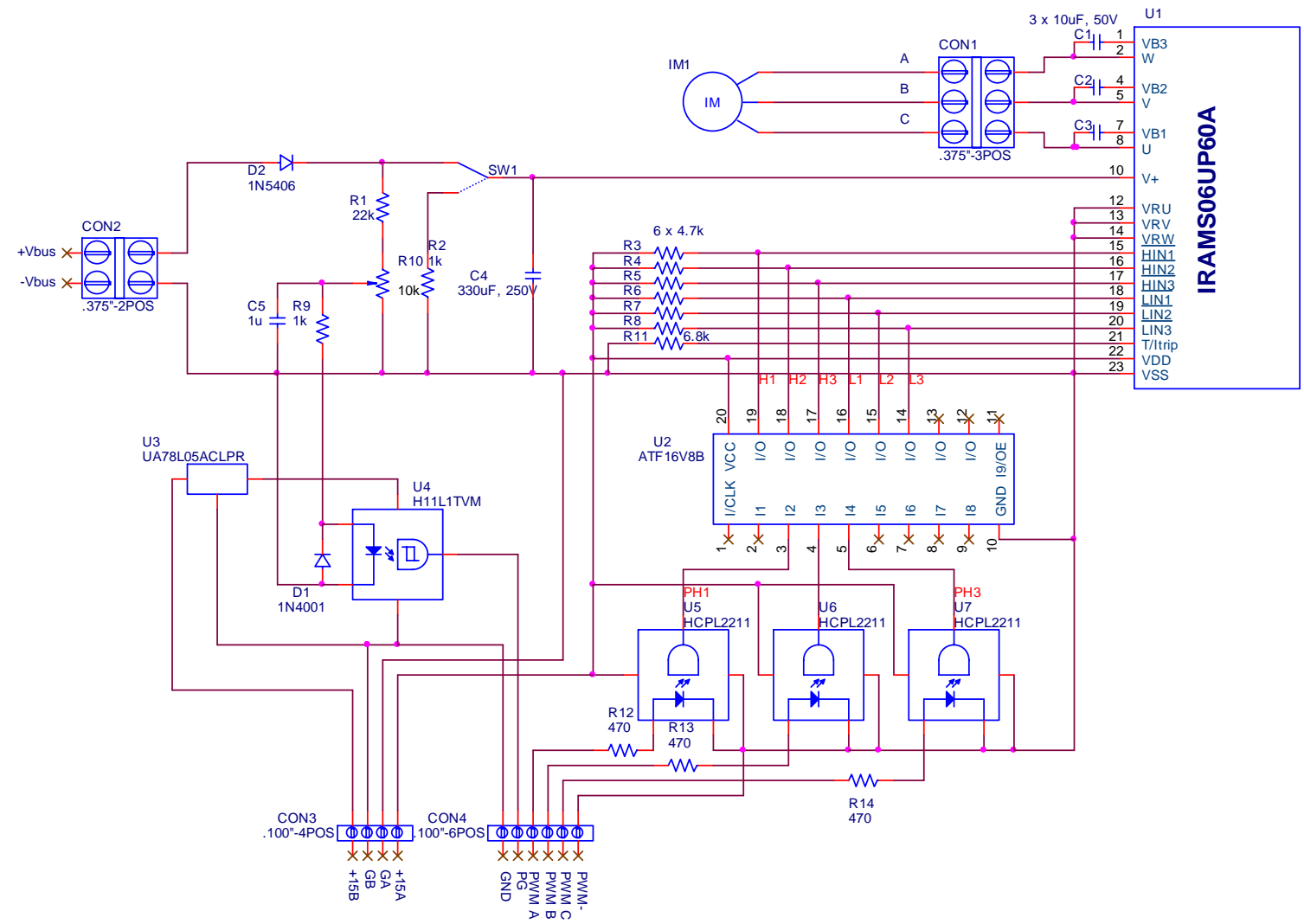

$3 \times 2 k, 20 W$, HLW-20-A1Z 2KOHM 5\% F01

Figure 23: Detailed Schematic of Motor Drive. 


\subsubsection{Waveform Generation and Speed Adjustment}

Synchronous speed is the speed of the magnetic field in the stator of an induction motor. For a three phase motor, which has $P$ number of pole pairs and is powered from a source of frequency $f$, synchronous speed $\omega_{s}$ is given by,

$$
\omega_{s}=\frac{120 \cdot f}{P}
$$

For an induction motor, rotor speed $\omega_{r}$ is always less than synchronous speed and the speed difference between rotor and stator is the slip.

$$
\omega_{s l}=\omega_{s}-\omega_{r}
$$

The slip depends on motor parameter such as the rotor resistance, and reactance, and load torque. Slip is inversely proportion to the square of supply voltage. An unloaded motor rotates at approximately synchronous speed. A variable frequency drive provides good speed adjustment for a low slip induction motor.

Variable frequency drive for the project offers frequency variation between 0 and $71 \mathrm{~Hz}$. A carrier frequency of $3.9025 \mathrm{kHz}$ is selected to allow up to 64 pulses per cycle for up to $61 \mathrm{~Hz}$. Selected sampling period of $64 \mu$ s allows up to 4 samples per PWM pulse.

Inverse Park Transformation is used to generate time varying 3-phase quantities from DC currents flowing in the two equivalent rotor windings $\mathrm{d}$ and $\mathrm{q}$. Winding axis directly falls on the axis of the virtual $d$ winding, and the $q$ winding on the quadrature axis.

$$
f_{a b c}=K \cdot f_{q d 0}
$$

Where,

$$
\begin{aligned}
& f_{d q 0}=\left[\begin{array}{lll}
f_{q} & f_{d} & f_{0}
\end{array}\right]^{T} \\
& f_{a b c}=\left[\begin{array}{lll}
f_{a} & f_{b} & f_{c}
\end{array}\right]^{T}
\end{aligned}
$$




$$
K=\frac{2}{3}\left[\begin{array}{ccc}
\cos \theta & \sin \theta & 1 \\
\cos \left(\theta-\frac{2 \pi}{3}\right) & \sin \left(\theta-\frac{2 \pi}{3}\right) & 1 \\
\cos \left(\theta+\frac{2 \pi}{3}\right) & \sin \left(\theta+\frac{2 \pi}{3}\right) & 1
\end{array}\right]
$$

And,

$$
\theta=\int \omega(\xi) d \xi+\theta(0)
$$

In order to generate three phase waveforms with fixed amplitude, direct axis can be chosen and quadrature axis can be set to zero. The magnitude and direction of the positive sequence component can be obtained by using equation (18).

$$
\left|V_{1}\right|=\sqrt{V_{q}^{2}+V_{d}^{2}} \quad, \quad \angle V_{1}=\tan ^{-1}\left(\frac{V_{q}}{V_{d}}\right)
$$

Setting $V_{q}=0$, the positive sequence components are determined by equation (19) [31].

$$
V_{a}=V_{d} \sin \theta, \quad V_{b}=V_{d} \sin \left(\theta-\frac{2 \pi}{3}\right), \quad V_{b}=V_{d} \sin \left(\theta-\frac{2 \pi}{3}\right)
$$

The Simulink model used to test performance of the variable frequency drive is shown in Figure 24.

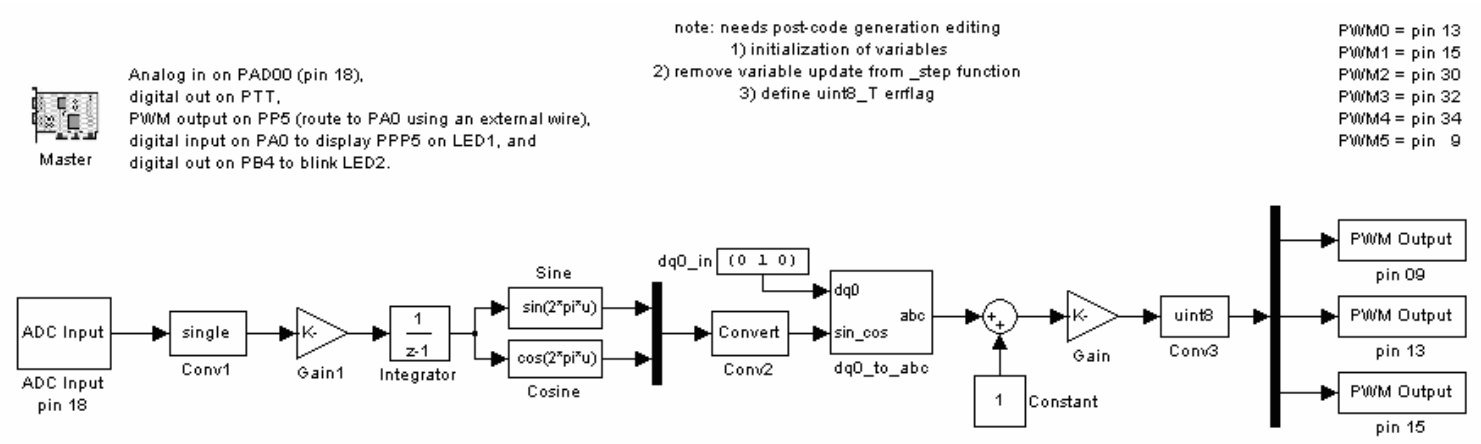

Figure 24: Simulink Model of Motor Drive Controller.

Analog input is used as the speed adjustment command. A combination of gain and integrator provides more than 64 step increments between 0 and $2 \pi$ for frequencies lower than $61 \mathrm{~Hz}$. 
The speed command is multiplied by the gain and integrated to produce angle for sine and cosine blocks. Output of cosine and sine blocks are used by the dq0 to abc block to generate a 3-phase signal. Sine and cosine blocks use normalized inputs and $2 \pi$ is represented by the value ' 1 '.

Table 5: Motor Controller Parameters.

\begin{tabular}{|l|r|}
\hline Available Parameter & Value \\
\hline Frequency Set-point & $0-255$ \\
\hline Direct axis equivalent DC & 1 \\
\hline Quadrature axis equivalent DC & 0 \\
\hline Zero sequence equivalent DC & 0 \\
\hline
\end{tabular}

\subsection{Pulsed Load}

The weaponry systems and radar onboard ships cause heavy pulse loading. The testbed also incorporates a pulse load to emulate the actual system. A Pulse Load (PL) is built around a constant current converter connected to a very low resistance load through capacitors. An additional high side MOSFET switch is added to facilitate the pulse load discharge command. The DC link between the converter and the switched load is connected to a bank of capacitors.

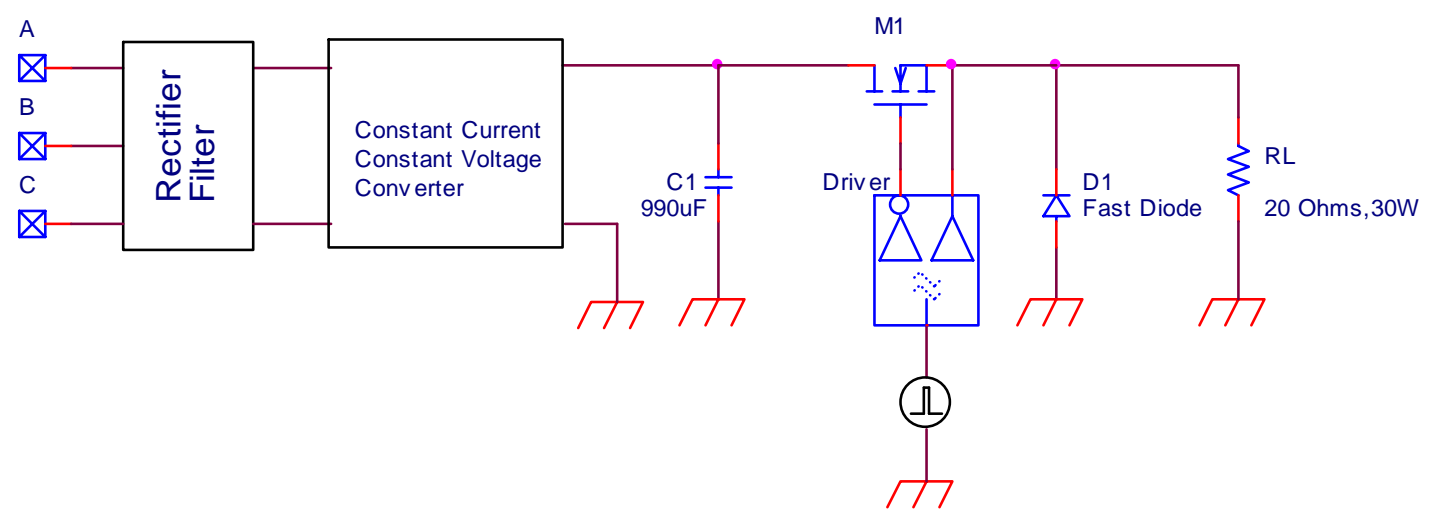

Figure 25: Line Diagram of Pulsed Load.

The converter topology used for the Pulsed Load is same as used for SSCM.

\subsubsection{Selection of Pulse Frequency}

The pulsed load is more than 5 times any individual zonal load. A converter is installed at the input of the PL which charges a capacitor bank at a constant current at the beginning of the charging cycle. When the voltage set-point of the converter is met, it maintains a 
constant voltage across the capacitor bank. Capacitor voltage can be obtained by integrating capacitor current over time. If the charging current is constant, voltage merely becomes a function of time. At any time $\mathrm{t}$, the voltage $V_{C}(t)$ across the capacitor $C$ charging at $I_{\text {Charging }}$ can be given by,

$$
V_{C}(t)=\left(\frac{I_{\text {Charging }}}{C}\right) \cdot t+V_{C}(0)
$$

Where $V_{c}(0)$ is the initial voltage of the capacitor. The selected $990 \mathrm{uF}$ capacitor can be charged up to $180 \mathrm{~V}$ in $890 \mathrm{~ms}$ if a constant charging current of $200 \mathrm{~mA}$ is applied. The voltage set-point being lower than $180 \mathrm{~V}$ occurs earlier than $890 \mathrm{~ms}$ and the converter switches into voltage regulation mode in the remaining time. Capacitor is supplied just enough current to meet the internal discharge and other leakage currents in the DC link during this mode.

Load switch, M1, turns on for $120 \mathrm{~ms}$, which exponentially discharges capacitor voltage until it reaches a value equal to $R \times I_{\text {Charging. }}$. Because the time constant for $20 \Omega$ load connected to the capacitor of $990 \mu \mathrm{F}$ is $22 \mathrm{~ms}$, the pulse discharge of $120 \mathrm{~ms}$ brings the link capacitor very close to a completely discharged state. If, for example, only a $10 \%$ voltage drop is desired for the same discharge time-span, it will require a $57 \mathrm{mF}$ capacitor. 


\subsubsection{Schematic}

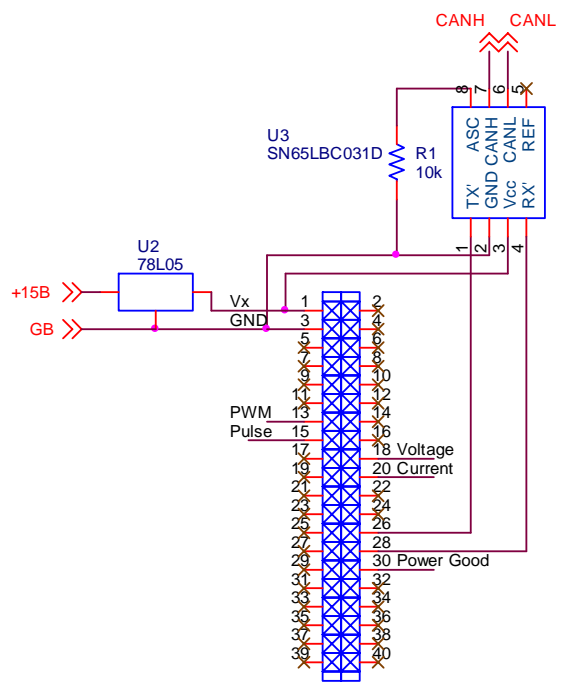

Figure 26: MC9S12C32 Plug-in Board for Pulse Load.

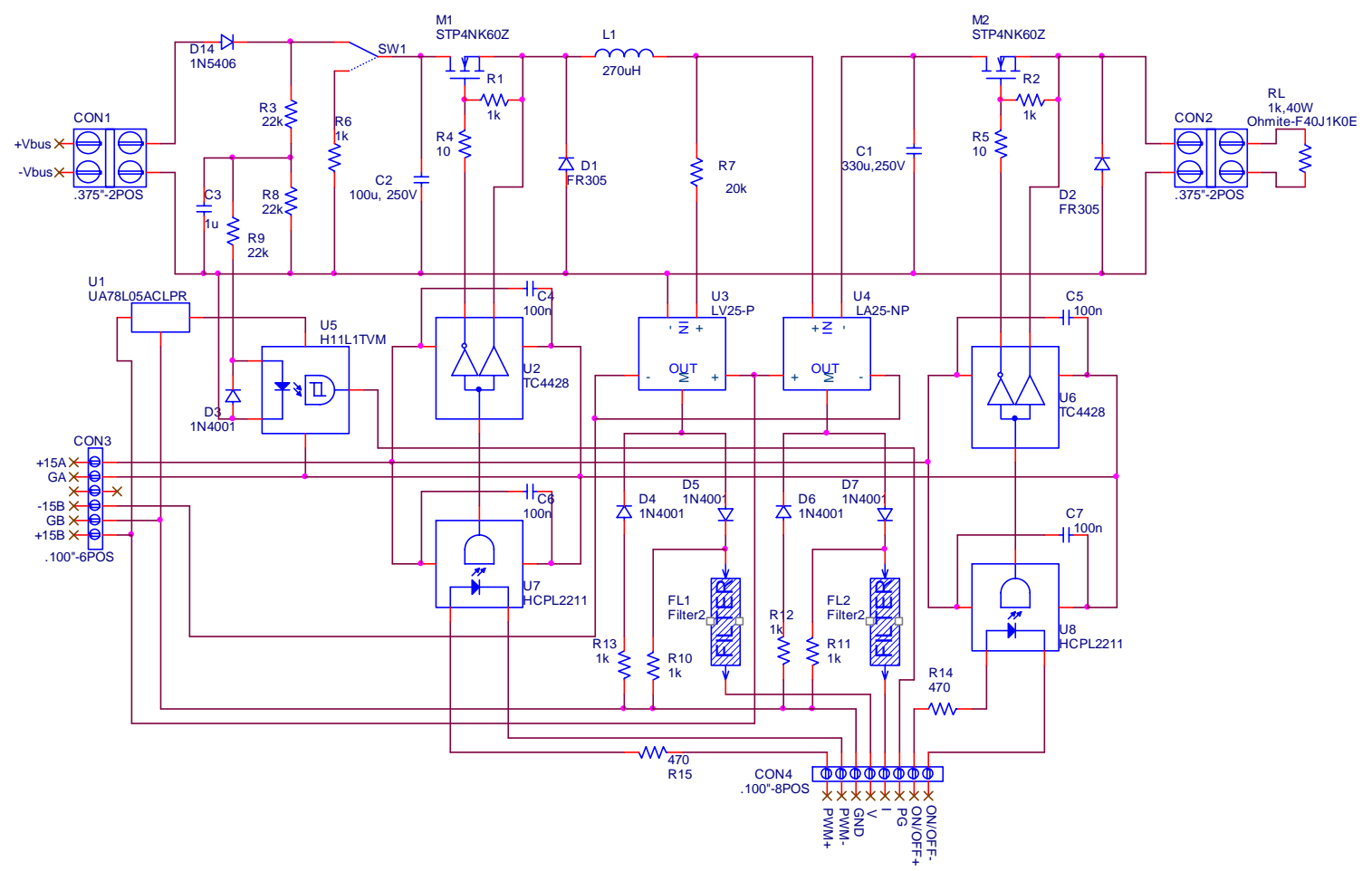

Figure 27: Detailed Schematic of Pulsed Power Load.

\subsubsection{Controller}

In addition to the voltage and current controller using PI control as well as the overload and short circuit protection a periodic signal for triggering the pulse load is required. The 
periodic switching pulse is generated using an integrator, which rolls over periodically. Figure 28 shows the Simulink model for the pulse generator. Voltage and current controls remain same as the power supply controller in section 3.3.9.

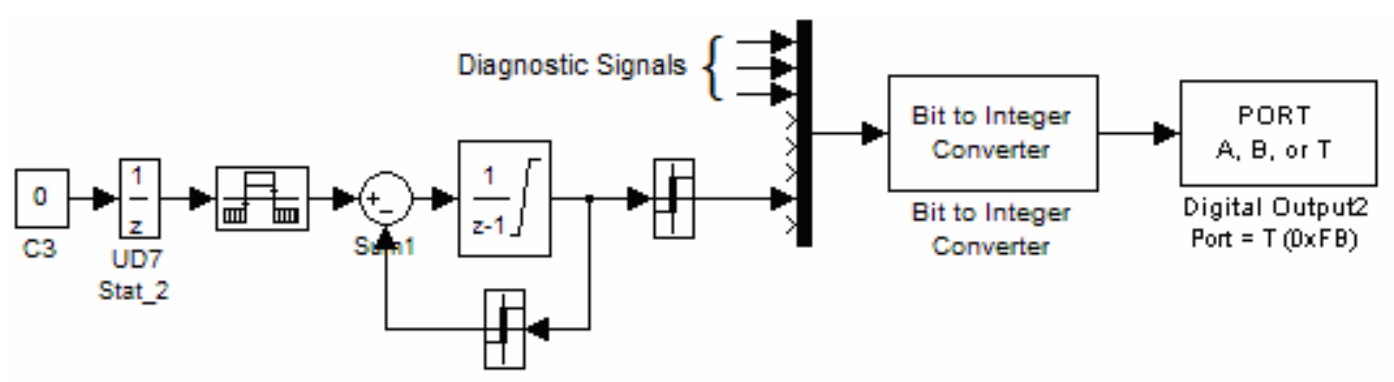

Figure 28: Simulink Model of Pulse Generator for Pulsed Load.

\subsection{Shipboard Service Converter Module}

Shipboard Service Converter Modules (SSCM) supply zonal buses of the shipboard power system. The design of this converter module is based on a non-isolated step-down DC-DC converter. It is built around a MOSFET based high-side switching chopper. Most of the design of the power section for the PS, described in section 3.3, is applicable to the SSCM.

SSCM, just like PS, adapts optical isolation gate drive and sensing circuits as shown in Figure 29.

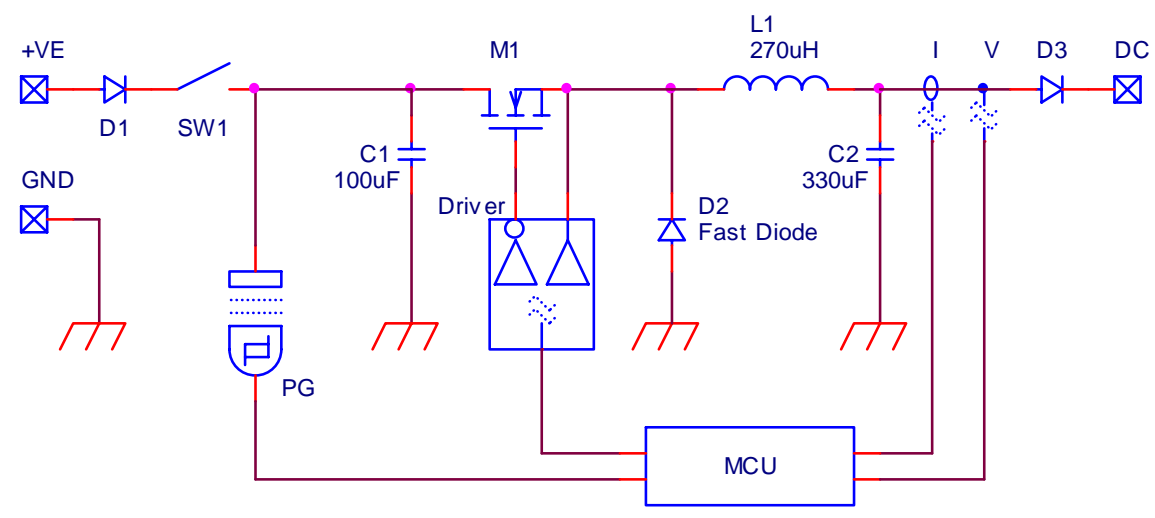

Figure 29: Line Diagram of Shipboard Service Converter Module.

This converter includes a diode at the output, which serves to block any backward flow of current in case of a higher voltage on the zonal bus. As the zonal bus is powered from converters on each of the two DC buses, there is a possibility that one converter is supplied from a higher voltage of another converter. 


\subsubsection{Selection of Output Filter Elements and Sensors}

As mentioned earlier, most of the designs of the PS are applicable to SSCM; a comparison has been made to see the effect of using similar component values. Using the same switching frequency as PS and a lower voltage difference between input and output in equation (3), a lower value inductance will be required for an SSCM. Whereas, keeping the same inductance value would further guarantee a continuous current mode operation. The ripple voltage of the output of a converter is proportional to the output ripple current. A reduced load current, when moving from PS to SSCM, assures smaller ripple voltage.

A current sensor with smaller maximum current can be used here. The same voltage sensor but with a lower current limiting resistor can be used for the SSCM.

\subsubsection{Circuit Breaker}

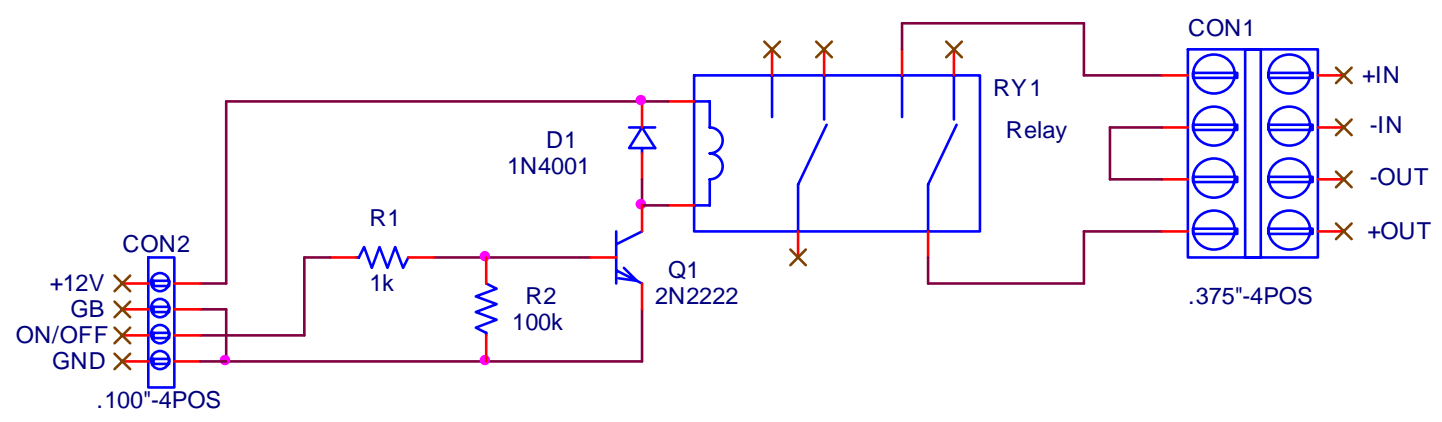

Figure 30: Detailed Schematic of Circuit Breaker.

Each converter module is associated with at least one breaker, which serves the purpose of connecting or disconnecting the DC line for reconfiguration purposes. Breakers are designed to be operated using a $5 \mathrm{~V}$ logic level signal. The breakers are based on electromechanical relays with a $12 \mathrm{~V}$ solenoid coil. The coil is connected between the positive power supply and the collector of the driving transistor [41]. Transistor acts like a current sink and logic high at the "ON/OFF" input, as shown in Figure 30, closes the contact while a logic low at the same input opens it. D1 is used to provide short circuit path to the back EMF generated during the turn-off process. 


\subsubsection{Schematic}

Figure 31 shows detailed schematic of the MCU board for SSCM. Converters SSCM4 and SSCM5 use both the breaker control outputs on pins 32 and 34 (see Figure 51 for device numbering). Remaining converters use breaker control output "a" on pin 32.

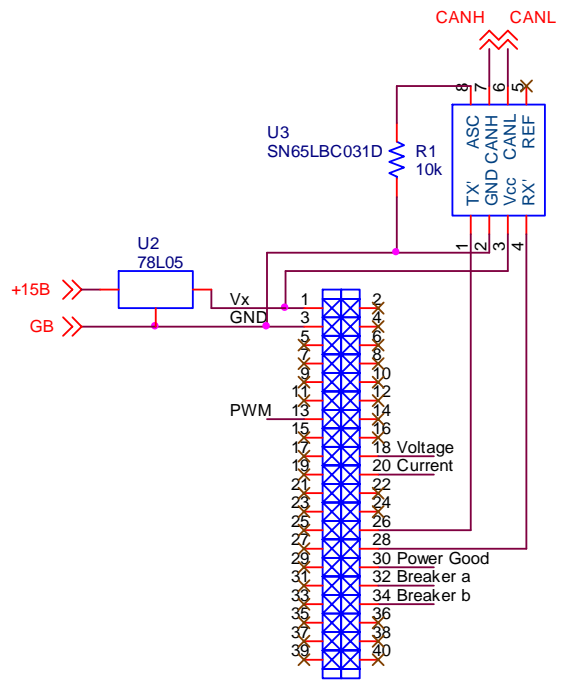

Figure 31: Detailed Schematic of MC9S12C32 Plug-in Board for SSCM.

Breaker controls are directly connected to circuit breaker board shown in Figure 30. 


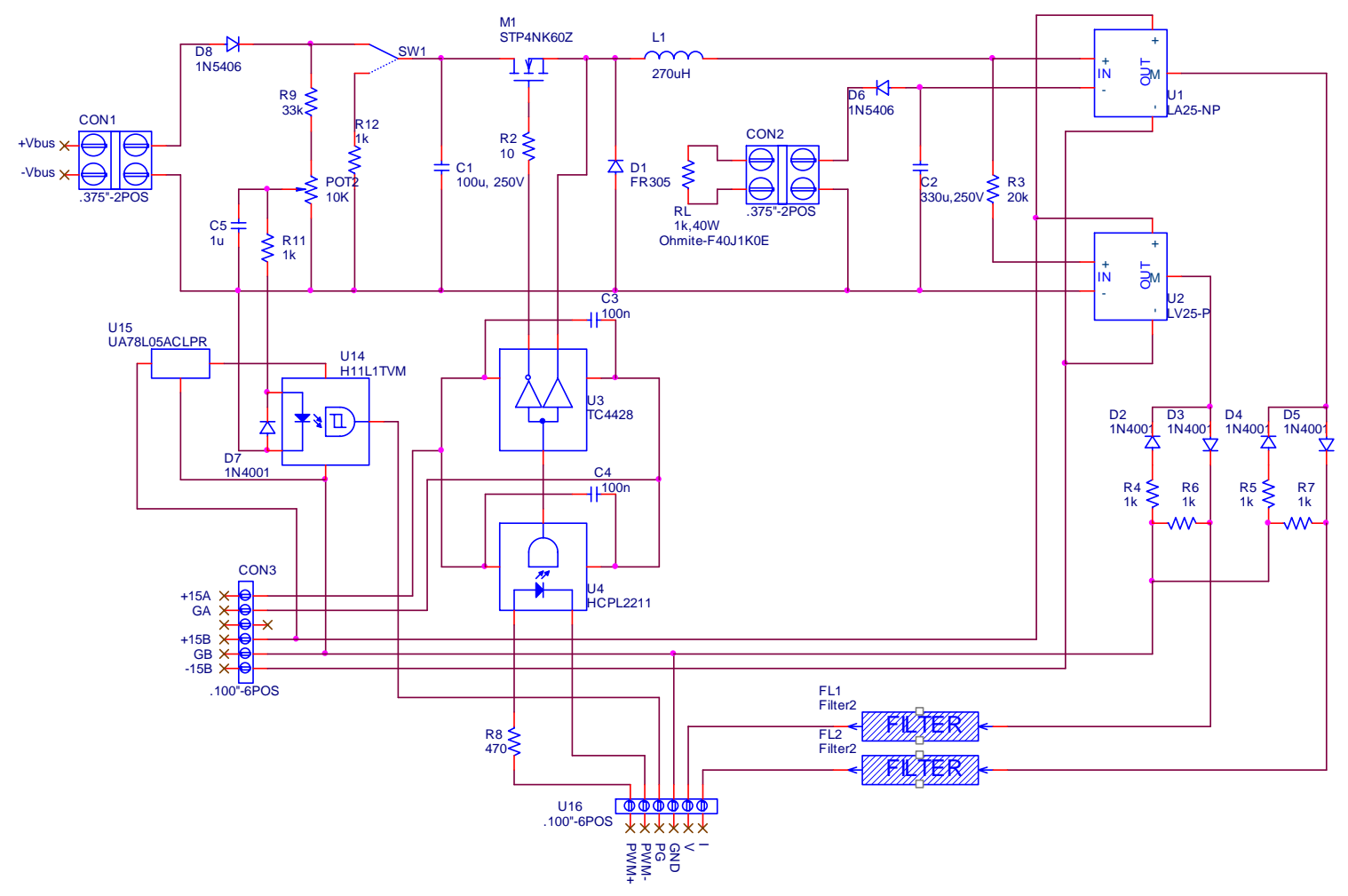

Figure 32: Detailed Schematic of Shipboard Service Converter Module.

\subsubsection{Controller}

Figure 33 shows the Simulink model of the power supply controls. Two versions of the converter modules are used. Four converter modules, which supply power to the first and the last zones, are connected to a single breaker. Remaining two modules, supplying the middle zone, control two breakers each. Inductor's current is adjusted by the inner controller to maintain the output voltage at the set-point. This is done by arranging output of the outer voltage controller as the reference for the inner controller. Overload and short-circuit protection have been implemented in the same way as for the Power Supply, described in section 3.3.9. Table 6 summarizes the parameters for a SSCM controller. 


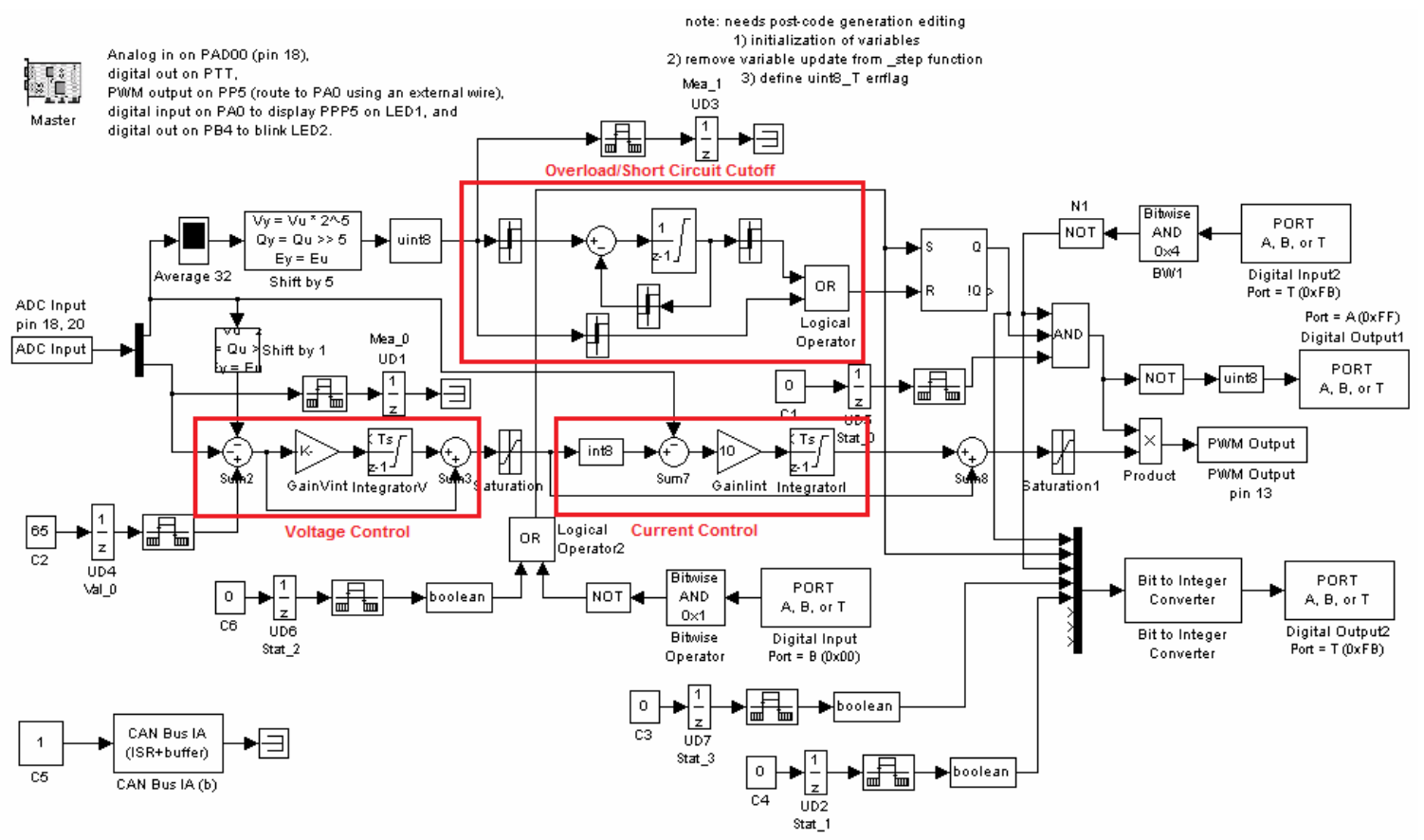

Figure 33: Simulink Model of Shipboard Service Converter Module.

Table 6: Shipboard Service Converter Module Controller Parameters.

\begin{tabular}{|l|r|}
\hline Available Parameter Name & Values \\
\hline Readable and Modifiable: & $0-1$ \\
\hline On-off mode flag for converter & $0-1$ \\
\hline On-off mode flag for first breaker & $0-1$ \\
\hline On-off mode flag for second breaker (some units only) & $0-1$ \\
\hline Overload/short circuit reset flag & $0-255$ \\
\hline Voltage set-point & $0-255$ \\
\hline Readable only: & $0-255$ \\
\hline Output voltage & 1 \\
\hline Load current & 100 \\
\hline Internal: & 10 \\
\hline Proportional gain of the PI voltage control & 255 \\
\hline Integral gain of the PI voltage control & 255 \\
\hline Integral gain of the PI current control & 0 \\
\hline Current set-point & 100 \\
\hline Controller saturation upper limit & 255 \\
\hline Controller saturation lower limit & 200 \\
\hline Overload cutoff limit & 8 \\
\hline Overload cutoff delay & 8 \\
\hline Short circuit cutoff limit & $0-1$ \\
\hline Voltage scaling factor &
\end{tabular}




\subsection{Constant Power Load}

Constant Power Load is a power regulating controller that uses power set-point as the command input. It is based on the same topology used by Power Supply described in section 3.3 and Shipboard Service Converter Module described in section 3.6.

\subsubsection{Controller Gain}

Constant Power Load control uses power set-point to generate the reference point for the current control loop. The following block diagram shows the basic function of the controller. Power set-point is divided by the voltage feedback to obtain current reference point. A lower limit is set at the voltage input to avoid division by zero. Gain at the power set-point input is intended at simplifying the controller design considering fixed point computation based on 8 bit integers. Power set-points can be entered as integer numbers between 0 and 15 , representing $0 \%$ to $100 \%$ of the full load.

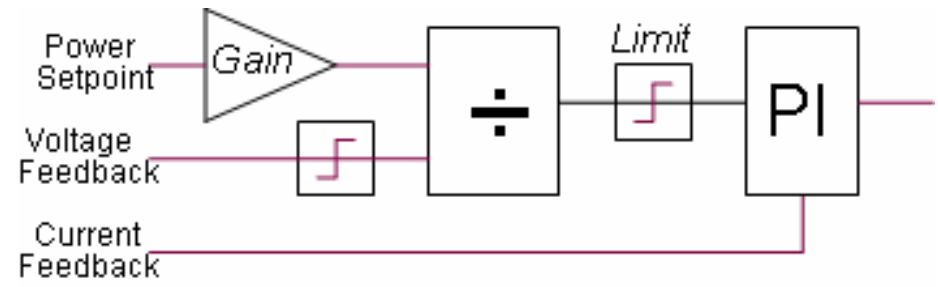

Figure 34: Constant Power Load Controller.

For $n$ number of power levels, spaced at equal amount of increments, gain can be obtained using the following equation,

$$
\text { Gain }=\frac{V^{2} \times 2^{2 b} \times r_{v} \times r_{i}}{n \times R \times v_{r}^{2}}
$$

Where, $b$ is the number of ADC bits, equal to 8 in this case. The gain of the voltage sensing circuit, $r_{v}$, is determined to be 0.06 and the gain of the current sensing circuit, $r_{i}$, is 5.0. $V$ is the maximum achievable load voltage, and $v_{r}$ is the reference voltage of ADC equal to $5.0 \mathrm{~V}$.

Considering load resistance, $R$, of $250 \Omega$, the gain equation can be re-rewritten as, 


$$
\text { Gain }=3.1212 \times\left(V^{2} / n\right)
$$

If the desired number of increments is set to 16 for the maximum achievable output voltage of $30 \mathrm{~V}$, required gain should be set at 176 .

Maximum current limit can be set at the maximum desirable load current by using the following relation.

$$
\text { Limit }=\frac{V \times 2^{b} \times r_{i}}{R \times v_{r}}
$$

Here the reference voltage for analog to digital converter, $v_{r}$, is 5 volts. Using available values, the equation simplifies to, Limit $=1.02 \times \mathrm{V}$. For the maximum attainable output voltage of $30 \mathrm{~V}$, limiter must be set to 31.0

\subsubsection{Controller}

Figure 35 shows the Simulink model for the constant power controller. The desired power-level is used as the input. This power level is converted to an internal value after multiplying it by the gain, division by the load voltage generates the desired current setpoint. Desired current at the load produces the commanded power for the Constant Power Load.

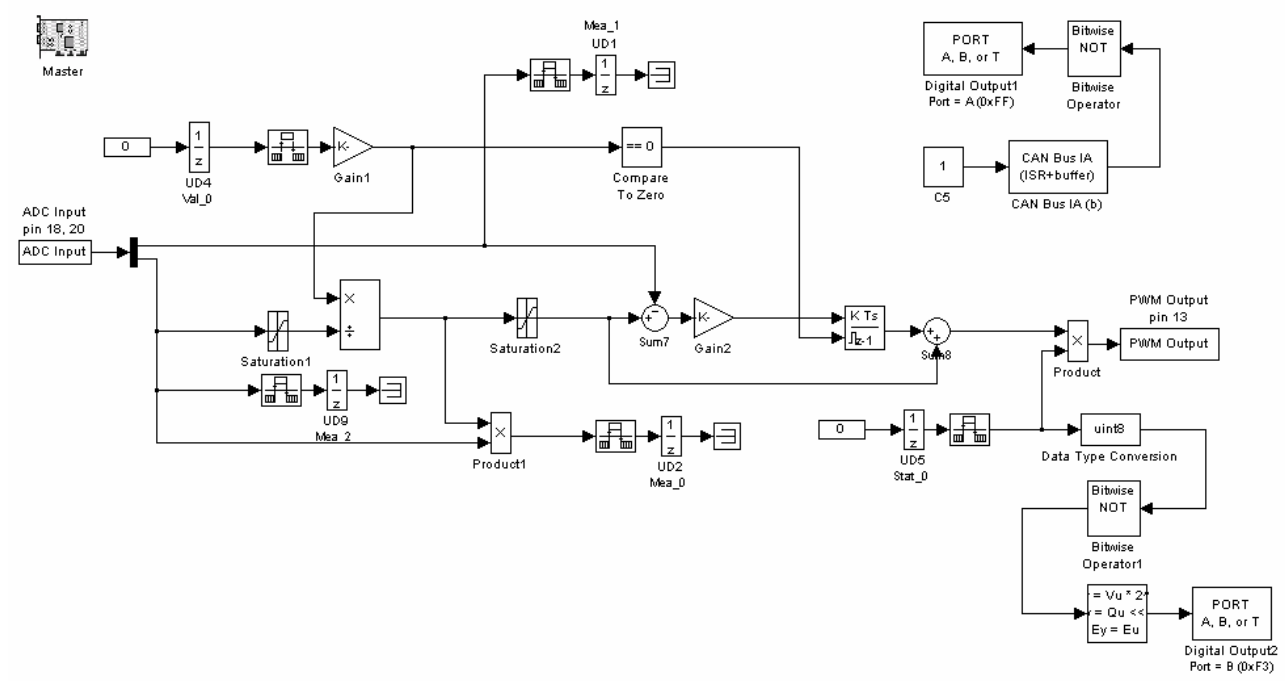

Figure 35: Constant Power Load Controller Simulink Model. 


\subsection{Internal Power Supply for Sensors and Drives}

As explained earlier, the cascaded nature of system integration among non-isolated converters as well as a non isolated communication bus makes it more difficult to connect single power source to supply overall power needs. The overall testbed is divided into two equal sections for the purpose of supplying power to the sensors and the drive circuits. The testbed contains a pair of dual power supplies to supply +15 and -15 volts power for sensors as shown in Figure 36.

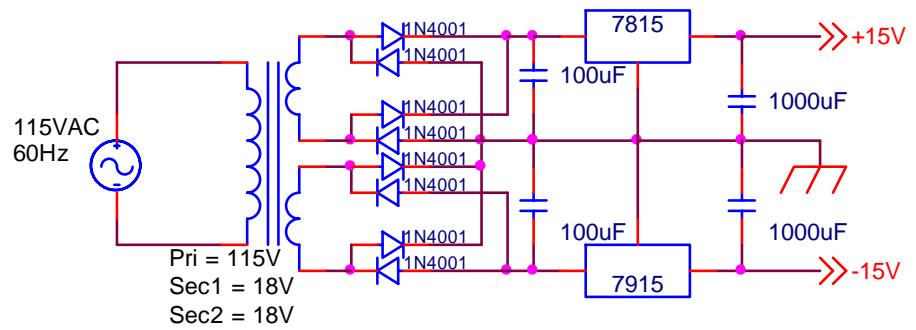

Figure 36: Power Supply for Gate Drives and Relays

A $5 \mathrm{~V}$ voltage regulator provides power to the microcontroller boards by converting the +15 volts side of the sensors' power supply.

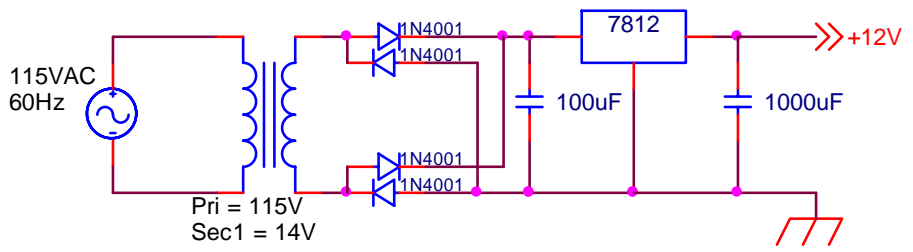

Figure 37: Power Supply for Sensor and MCU

Separate $12 \mathrm{~V}$ power supplies provide power to the gate drives of the converters in those two sections of the testbed. An additional $12 \mathrm{~V}$ power supply is added to the testbed to provide power to the eight breakers. 


\subsection{Communication}

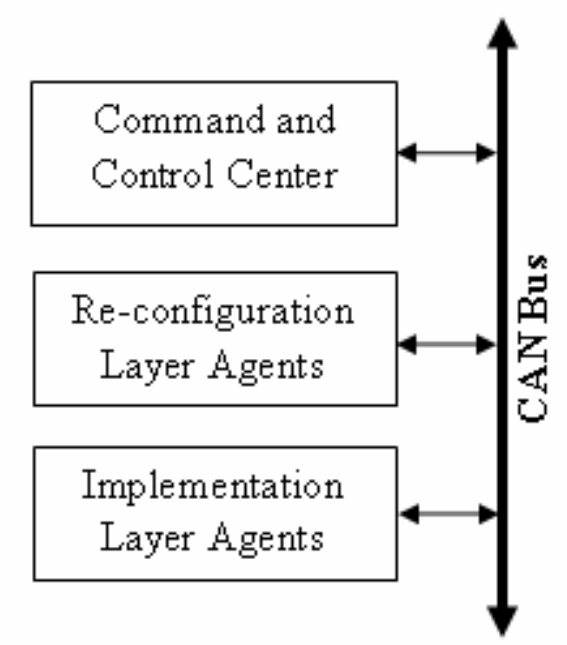

Communication forms the most important part of the agent based control. Agents must communicate with their neighboring counterparts to achieve local as well as global objectives. A robust communication is desired for the testbed because all the supervisory controls reside in the re-configuration layer or the command and control center. The testbed consists of power electronics and induction motor drives and RFI or EMI generated at the testbed must not hamper communication.

Each agent in the implementation layer teams up with its counterpart in the reconfiguration layer. Implementation Agents (IA) and Reconfiguration Agents (RA) have been described in the introduction of Chapter 3. A peer-to-peer communication is required between each IA and RA team. Command and control center, IA and RA connect to each other through a common communication bus and using a common messaging language. These entities need to communicate with each other at different modes of operation. For example, a new flow-value obtained by running algorithms in the reconfiguration layer is sent to the implementation agents in the testbed and the command and control center must be able to read and write values to or from the testbed. Unique addresses must be assigned to each of these agents in order to have them perform these operations. These agents must also use the same standard for both the addressing scheme and the message format. The following sub-sections present the addressing scheme and message formats in more details. 


\subsubsection{Addressing}

In a Controller Area Network (CAN), arbitration identifies a message and also provides prioritization. The message with the lowest binary value in the arbitration field gets the highest priority on the bus and other messages wait until high priority messages are completely sent. A collision between two or more messages is resolved by using nondestructive arbitration. CAN in its original form does not carry any information about the sender. However, an agent must send query messages, which must include the sender's information so that the reply can be targeted at the original sender. Several protocols are available and these have been mentioned in Chapter 2. Communication standard developed by Foundation for Intelligent Physical Agents (FIPA) and CANopen have been adapted for the communication scheme of reconfiguration agents [44], and the hardware testbed also uses this messaging structure.

The FIPA Agent Communication Language (ACL) sets forth message parameters. Other than mandatory performatives, use of these parameters entirely depends upon the application. Sender, Receiver, and Content information are expected to be present in all ACL messages [17].

The first four bits out of the 29 bit extended identifier are set aside as message priority bits. Last bit in the identifier is reserved for multi-frame messages. Remaining 24 bits are divided into groups of 12 bits. Higher 12 bits are allocated for sender address and lower 12 bits are allocated for receiver address.

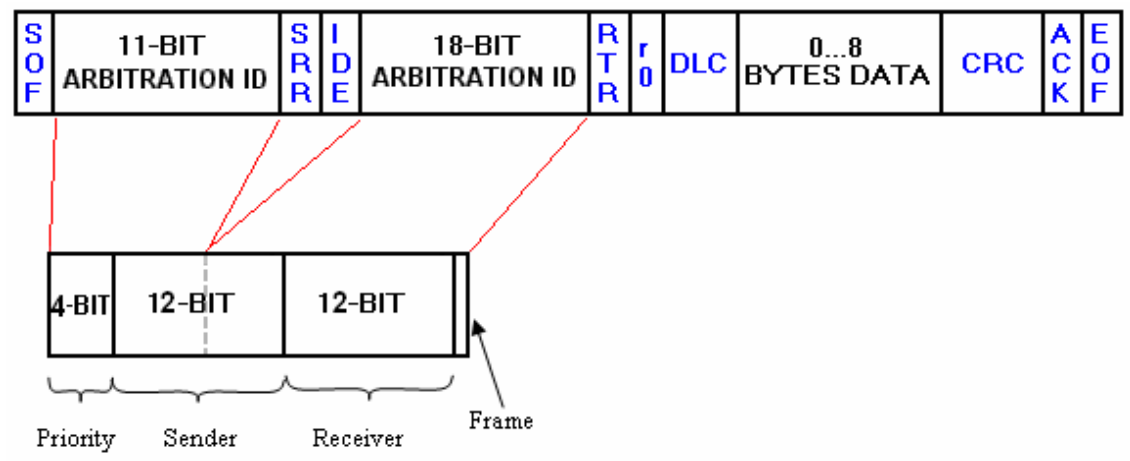

Figure 38: CAN Message Format Using Sender's and Receiver's Address.

Each node in the system is assigned a 12-bit binary address. As many as 4096 entities are possible with 12 bit addressing scheme. The testbed currently contains a total of 27 
entities including motor drives, converters, loads, and breakers within the testbed. Breakers are not assigned their own addresses but considered to be part of the SSCMs. Therefore this scheme leaves sufficient room for future expansion, e.g., addition of other agents.

Table 7 lists the addresses assigned to the devices on the testbed using address for RA/IA. Least significant bit is set to recessive if the message has multiple frames and to dominant if the message is composed of a single frame.

Table 7: Addresses for Testbed Components.

\begin{tabular}{|c|c|c|c|}
\hline & Device & Single Frame & Multiple Frame \\
\hline 1 & Power Supply 1 & $\mathrm{XXXXX \odot \odot 4H}$ & XXXXX๑๑5H \\
\hline 2 & Power Supply 2 & $\mathrm{XXXXX \odot \odot 2H}$ & XXXXX๑๑3H \\
\hline 3 & Shipboard Service Converter Module 1 & $\mathrm{XXXXX \odot 10H}$ & 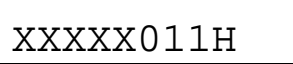 \\
\hline 4 & Shipboard Service Converter Module 2 & XXXXX๑७EH & $\mathrm{XXXXX \odot \odot FH}$ \\
\hline 5 & Shipboard Service Converter Module 3 & $\mathrm{XXXXX \odot \odot CH}$ & 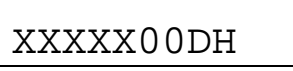 \\
\hline 6 & Shipboard Service Converter Module 4 & 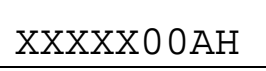 & XXXXX๑९BH \\
\hline 7 & Shipboard Service Converter Module 5 & XXXXX००8H & XXXXX००9H \\
\hline 8 & Shipboard Service Converter Module 6 & $\mathrm{XXXXX \odot \odot 6H}$ & $\mathrm{XXXXX \odot \odot 7H}$ \\
\hline 9 & Constant Power Load 1 & $\mathrm{XXXXX01 \textrm {AH }}$ & $\mathrm{XXXXX01BH}$ \\
\hline 10 & Constant Power Load 2 & $\mathrm{XXXXX \odot 18H}$ & $\mathrm{XXXXX \odot 19H}$ \\
\hline 11 & Constant Power Load 3 & $\mathrm{XXXXX016 \textrm {H }}$ & 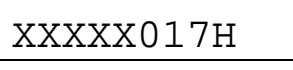 \\
\hline 12 & Constant Power Load 4 & 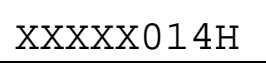 & XXXXX@15H \\
\hline 13 & Constant Power Load 5 & $\mathrm{XXXXX012 \textrm {H }}$ & $\mathrm{XXXXX \odot 13H}$ \\
\hline 14 & Pulsed Load 1 & 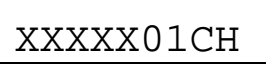 & XXXXX01DH \\
\hline 15 & Motor Controller 1 & XXXXX०1EH & XXXXX01FH \\
\hline 16 & Sensor 1 & XXXXX०2०H & $X X X X X \odot 21 H$ \\
\hline 17 & Propulsion 1 & 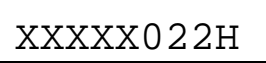 & $\mathrm{XXXXX \odot 23H}$ \\
\hline 18 & Propulsion 2 & $\mathrm{XXXXX \odot 24H}$ & 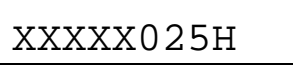 \\
\hline 19 & Sensor 2 & $X X X X X \odot 26 H$ & $X X X X X \odot 27 H$ \\
\hline
\end{tabular}




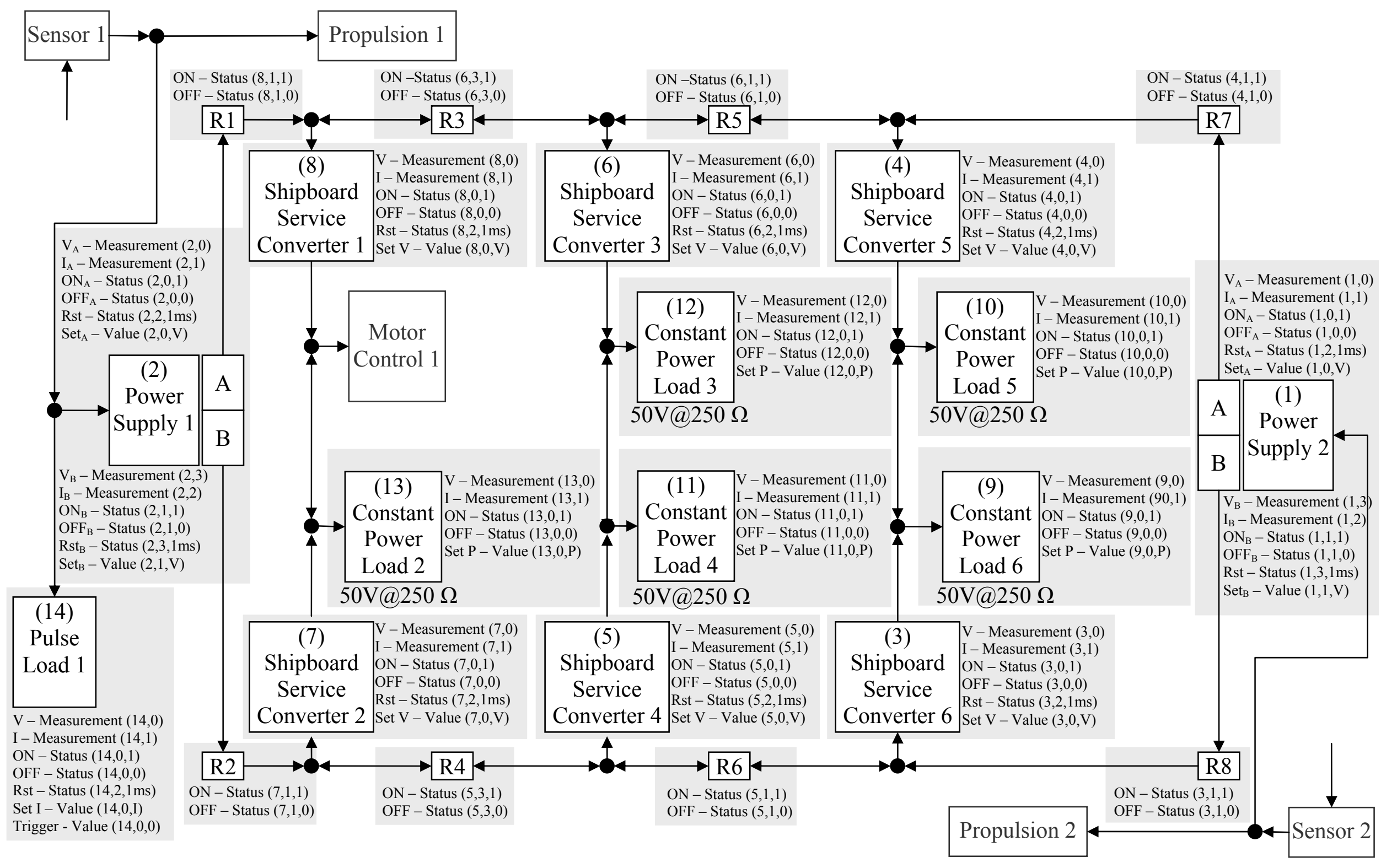

Figure 39: Addresses and Command Summary 


\subsubsection{Messages}

CAN frame provides 8 bytes for sending data. Messages are designed to send information to the implementation agents and receive information from the implementation agents. The messages exploit the performatives specified in the FIPA standard using a bit efficient protocol [18][49]. All the mandatory performatives are implemented using a part of the data field. The description below elaborates on different fields used in the messages.

Frame ID: first byte in the data field, which represents one of the 256 frames sent as a multi-frame message.

From addr: first 4 bits of the second byte in the data field. This represents the address of the process where the message originated.

To addr: second 4 bits of the second byte in the data field. This represents the address of the process to which the message is targeted.

Message type: third byte in the data field, which represents the relevant action to be taken on the data.

Data type: first four bits of the fourth byte in the data field. This represents the nature of the data sent.

Scaling: last four bits of the fourth byte in the data field. Scaling is used when delivering real world values through eight data bits only, more details in section 3.9.3.

Data type classification: fifth byte in the data field. This field further classifies data specified by the first four bits of the fourth byte.

Designator: sixth byte in the data field, which represents one among several of the data types relevant to a device.

Data: seventh byte in the data field, which carries the numeric value part of the message.

\subsubsection{Number Representation}

A real number can be represented by two groups of binary numbers. One group represents the whole number part and the other group represents the fraction part of the real number. Significant figures in the original number should be preserved while making 
the new representation. The Hall-effect voltage and current sensors used in this testbed have approximately the same accuracy and hence fixed digits of significance can be used for all the measurements on the testbed. Voltages and current values for different devices are of different magnitude. Different scaling factors with their precision and range are summarized in Table 8 below [2]. The formats use three bits out of 4 bits available for scaling in the original message shown in Table 10 on page 58. The forth and the most significant bit is used to indicate that the range of the value is above 255 as shown in Table 9.

Table 8: Binary Fractional Number Representation.

\begin{tabular}{|c|c|c|c|r|r|}
\hline Scaling & Format & Integer bits & Fraction bits & Precision & \multicolumn{1}{c|}{ Range } \\
\hline 1000 & 8.0 & 8 & 0 & 1 & $0-255$ \\
\hline 0000 & 7.1 & 7 & 1 & 0.5 & $0-127.5$ \\
\hline 0001 & 6.2 & 6 & 2 & 0.25 & $0-63.75$ \\
\hline 0010 & 5.3 & 5 & 3 & 0.125 & $0-31.875$ \\
\hline 0011 & 4.4 & 4 & 4 & 0.0625 & $0-15.9375$ \\
\hline 0100 & 3.5 & 3 & 5 & 0.03125 & $0-7.96875$ \\
\hline 0101 & 2.6 & 2 & 6 & 0.015625 & $0-3.984375$ \\
\hline 0110 & 1.7 & 1 & 7 & 0.0078125 & $0-1.9921875$ \\
\hline 0111 & 0.8 & 0 & 8 & 0.00390625 & $0-0.99609375$ \\
\hline
\end{tabular}

Formats can be chosen from the table to suit precision and range requirements of the application. As an example, $0.5 \mathrm{~A}$ current sensor, with an accuracy of $1 \%$, demands a resolution of $5 \mathrm{~mA}$. For representing it without losing precision, a 0.8 format binary number will be suitable, which also gives the required range in this case. As another example, if the value to be represented ranges up to 100 volts, a 7.1 number format will be suitable as it provides $0.5 \mathrm{~V}$ resolution.

If the most significant bit out of the 4-bit scaling nibble contains a ' 1 ' then the three lower bits represent the value of the multiplier. In this case, a 0 in those three bits indicate a multiplier of value ' 1 '. A 7 indicates the value of multiplier equal to ' 8 '. A maximum RPM of 2000 can be represented by using 7 in these three bits. This can represent up to a numbers 2040. The following table summarizes how the scaling can be used to represent numbers larger than 255. In this case the precision of the represented number may be lost, on the values sent over the communication link. The controller, which runs directly on 
the local MCU, does not see any loss in the precision as it uses the internally stored original values.

\begin{tabular}{|c|c|c|c|}
\hline Scaling & Multiplier & Precision & Range \\
\hline 1000 & 1 & 1 & $0-255$ \\
\hline 1001 & 2 & 2 & $0-510$ \\
\hline 1010 & 3 & 3 & $0-765$ \\
\hline 1011 & 4 & 4 & $0-1020$ \\
\hline 1100 & 5 & 5 & $0-1275$ \\
\hline 1101 & 6 & 6 & $0-1530$ \\
\hline 1110 & 7 & 7 & $0-1785$ \\
\hline 1111 & 8 & 8 & $0-2040$ \\
\hline
\end{tabular}

Table 9: Larger Number Representation 
The following table summarizes message data fields.

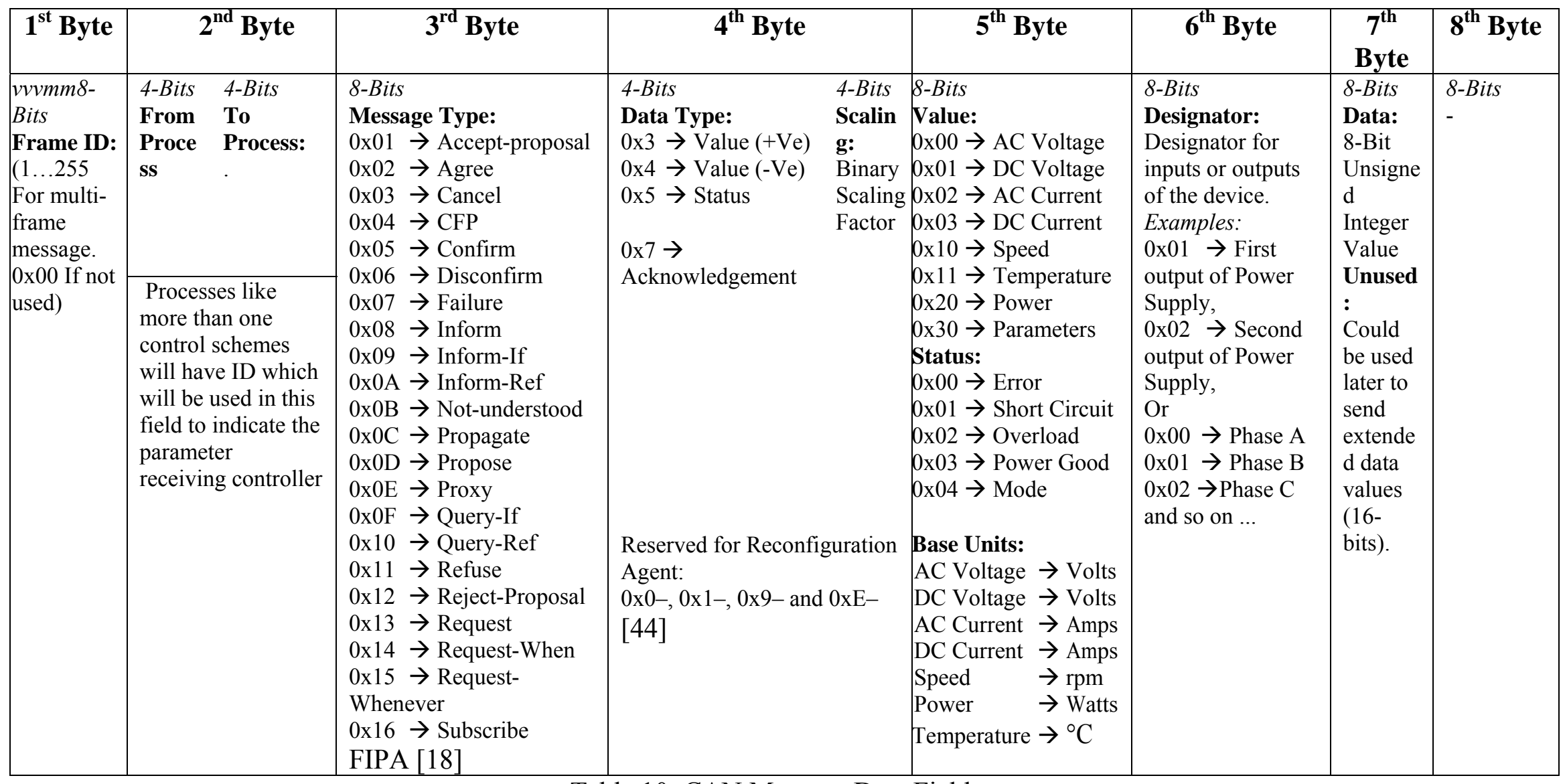

Table 10: CAN Message Data Field. 


\subsection{User Interface for Testbed Monitoring}

A remote computer terminal has been developed to provide a graphical user interface for monitoring activities of the various devices and also verifying performance. The console is built using a MATLAB Graphical User Interface (GUI). This user interface provides access to all the commands as well as measured quantities in an easy to use integrated console using color codes.

Individual devices on the testbed can be enabled, disabled, or reprogrammed with new set-point values. The initialization function configures testbed components to send measurement values periodically. A timer based function periodically reads the USB-toCAN converter's buffer and displays them in the appropriate locations on the user interface screen. This interface provides direct access to the converter set-points and a 16 level pull-down menu for setting the constant power loads' demand levels.

The interface also makes available status and measurement values in the form of a matrix accessible within the workspace. This matrix, saved as status in the workspace, is periodically updated using a timer function. This matrix can be shared with other entities like the reconfiguration algorithm or the command and control center. The matrix can also be used for further analysis.

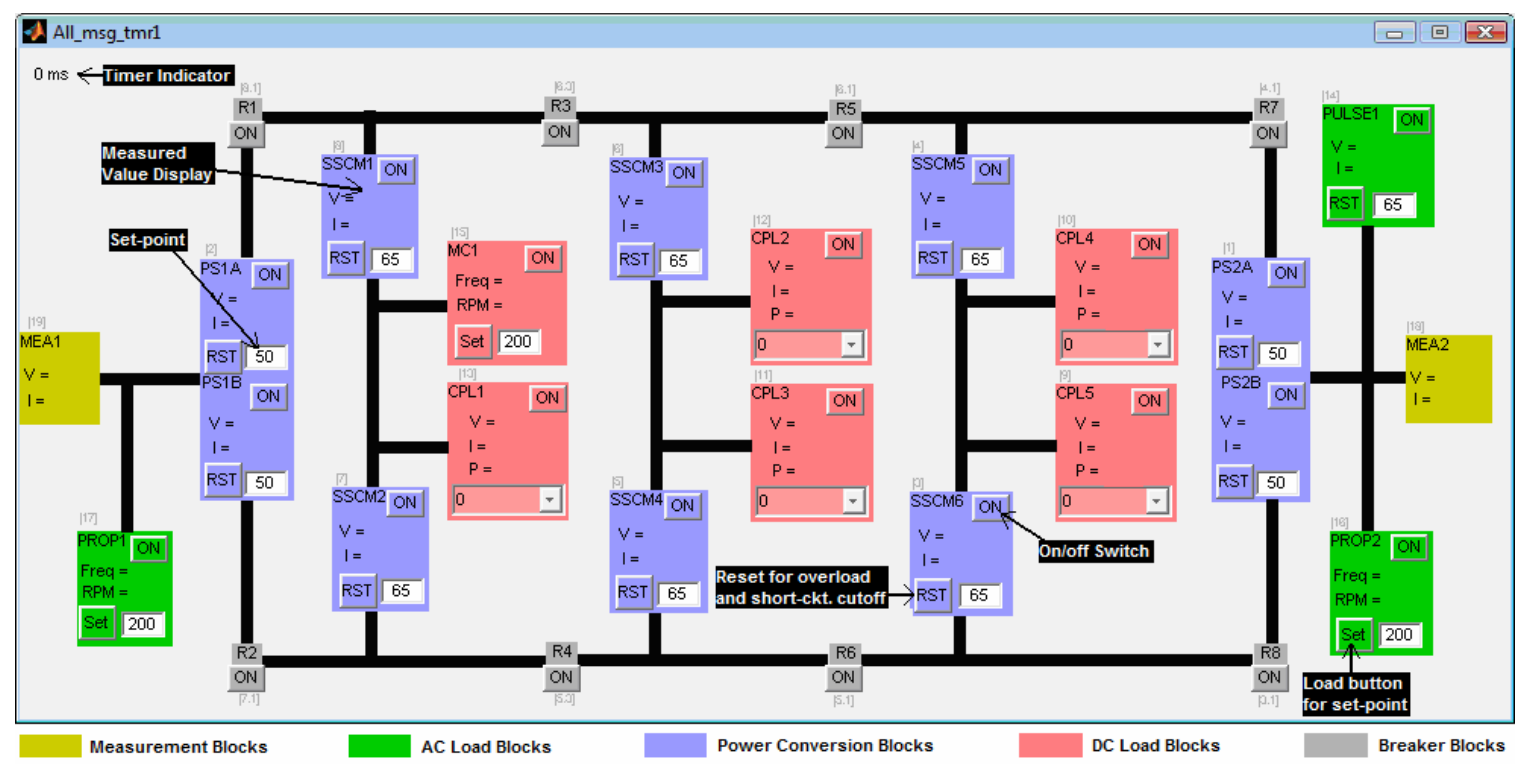

Figure 40: A Snapshot of the User Interface. 


\subsubsection{GUI Functions}

Each CAN node provides access to one or more variables. The number of available variables at a specific CAN node are set by the initialization function (Figure 40) and stored in vector $\boldsymbol{c a n} \_\boldsymbol{m} \_\boldsymbol{b}$. This vector allows the variables to be addressed in a loop using indices.

The 'timer' object has a number of properties that can be created and defined using the 'timer(') function. 'TimerFcn' sets function to be executed at a fixed rate of 'Period' seconds. BusyMode and ExecutionMode define different modes of execution. handles.c and handles.n store data in the GUI's handles structure. The initialization also creates a state matrix with 27 rows and 16 columns, which saves the state of different status blocks as well as measurement values in the form of a matrix shown in Table 11.

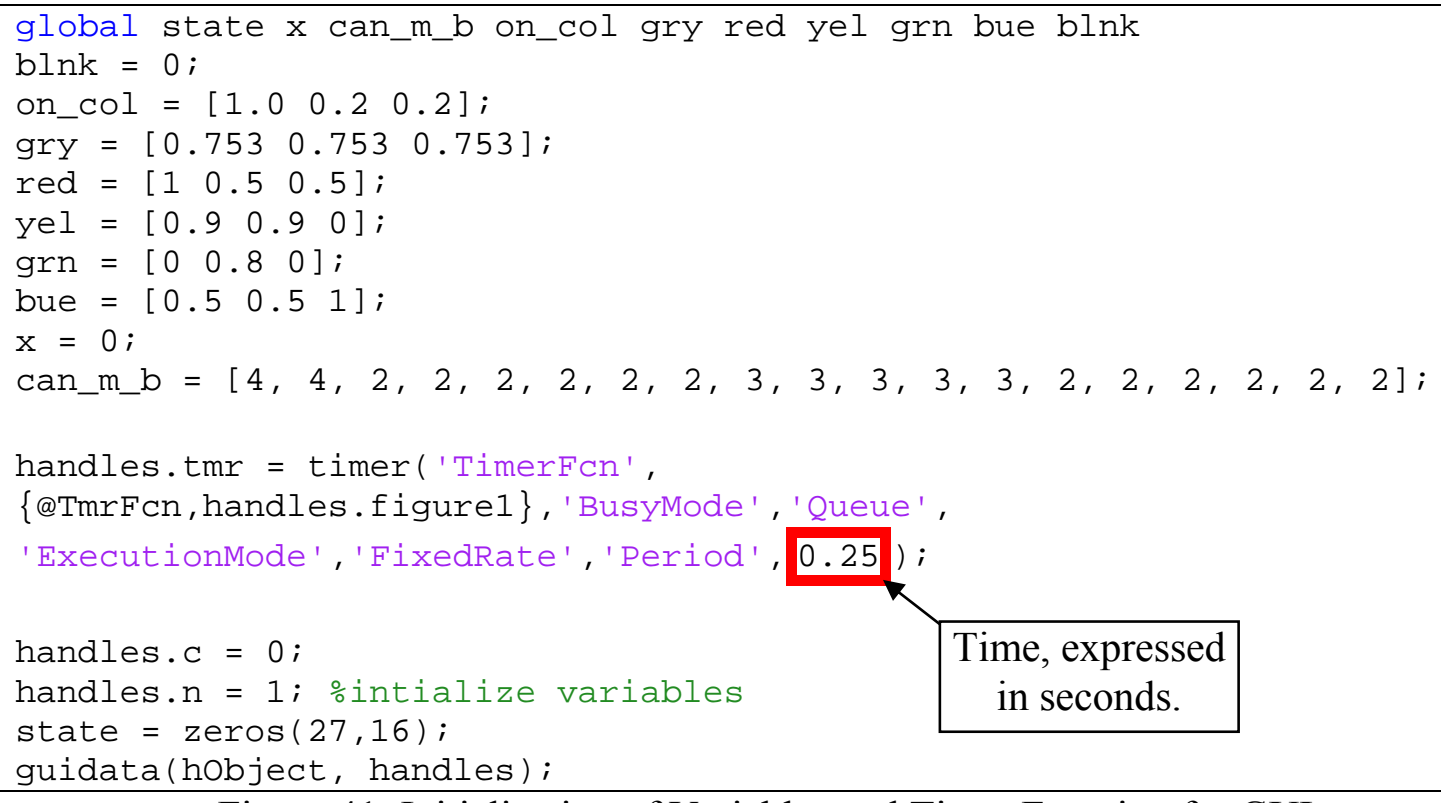

Figure 41: Initialization of Variables and Timer Function for GUI

First 'for' loop (Figure 41) uses 'can_m_b' to sequentially initializes measurement blocks to send periodic data. Second 'for' loop disables this feature for each block.

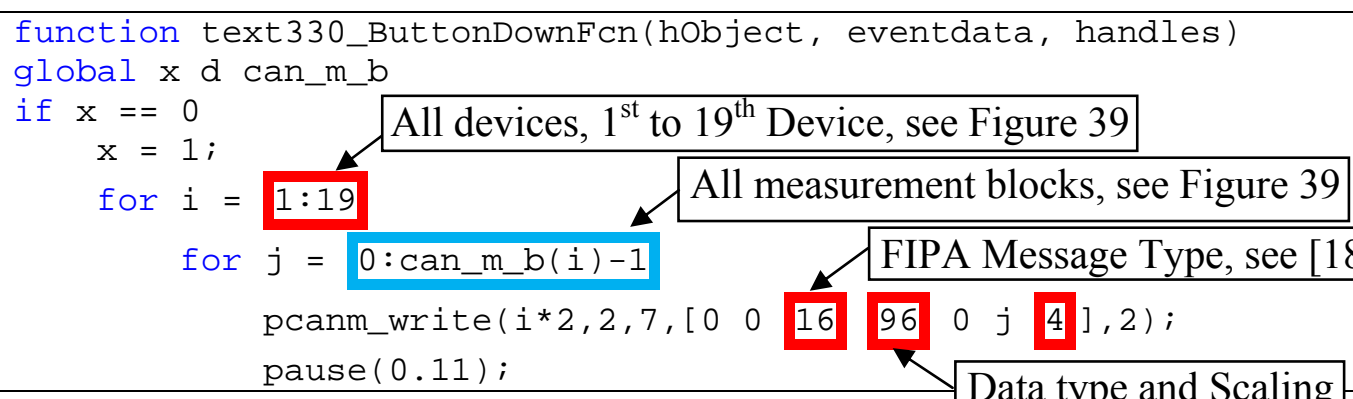




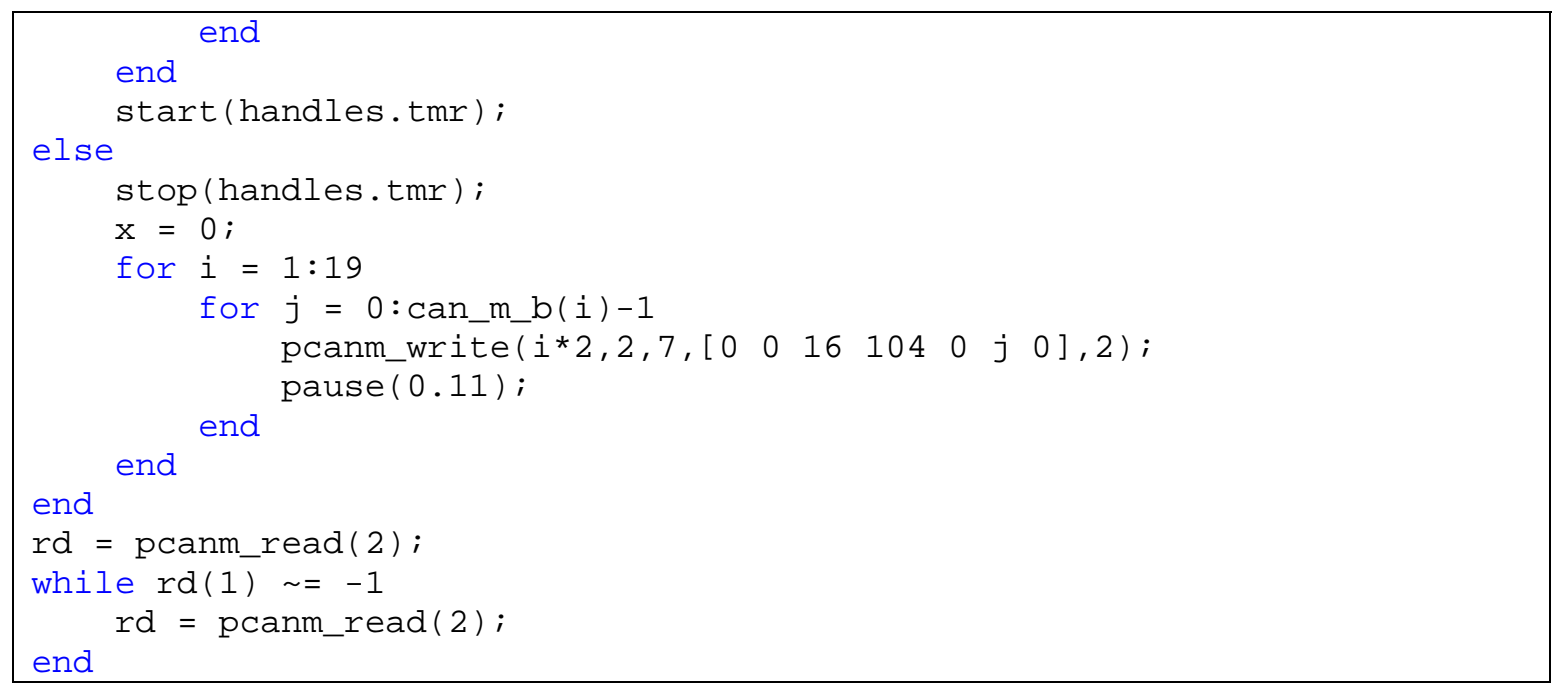

Figure 42: Initialization Function for Periodic CAN Messaging

Figure 43 below is an example of a function for powering on and off a converter module or a relay. The corresponding element in state is checked and the state is toggled, the resulting value is saved in the matrix.

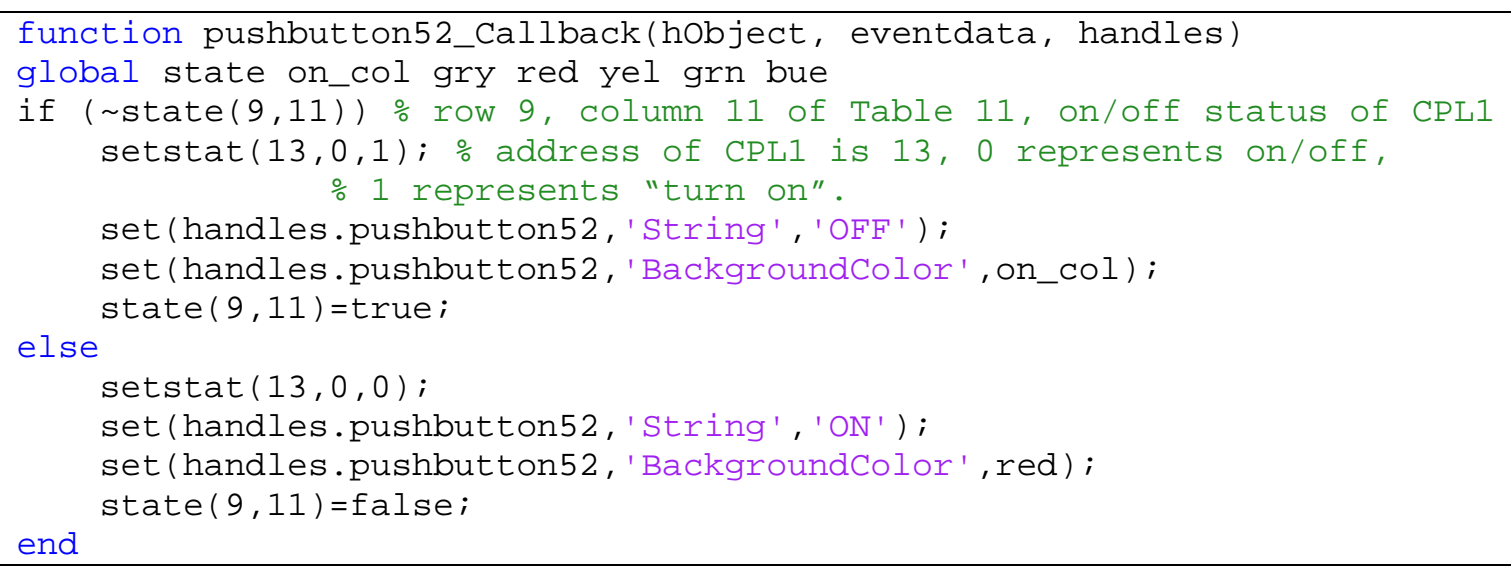

Figure 43: GUI Function for Turning “On” and “Off” Entities on the Testbed

\subsubsection{Commands}

All the commands for the testbed have been summarized in Figure 39. SPS testbed commands can be broken into inform and query. The query commands are used to request information from IAs. The inform commands are used to pass information like the set-point, status or the measurement values over the network. 
Table 11: Elements of state Matrix.

\begin{tabular}{|c|c|c|c|c|c|c|c|c|c|c|c|c|c|c|c|c|c|c|}
\hline & 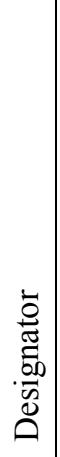 & 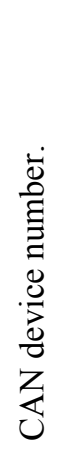 & 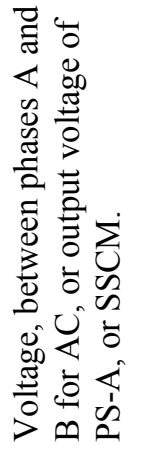 & 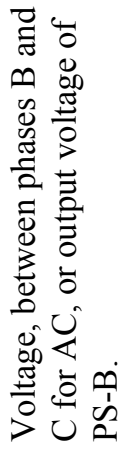 & 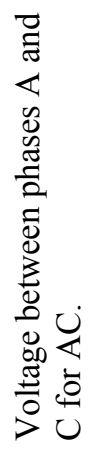 & 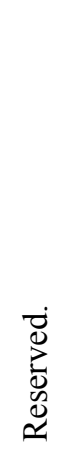 & 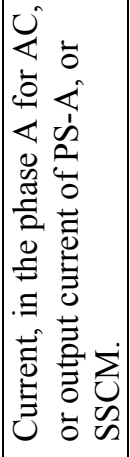 & 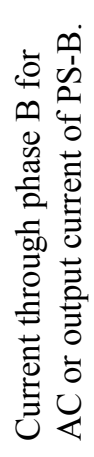 & 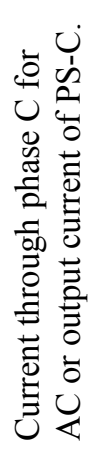 & 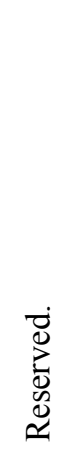 & 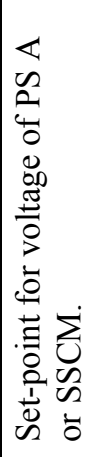 & 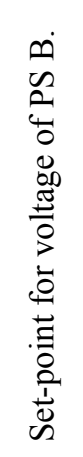 & 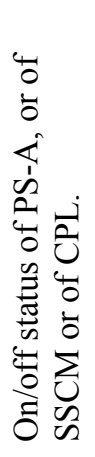 & 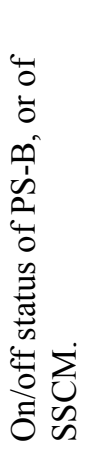 & 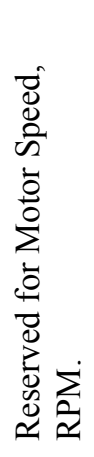 & $\begin{array}{l}\overrightarrow{0} \\
0 \\
\text { a } \\
\overrightarrow{0} \\
0 \\
0 \\
\overrightarrow{0} \\
0 \\
0 \\
0\end{array}$ & 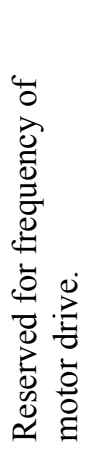 & 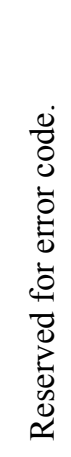 \\
\hline \multirow{2}{*}{ Power Supply } & 2 & 1 & $(1,1)$ & $(1,2)$ & $(1,3)$ & $(1,4)$ & $(1,5)$ & $(1,6)$ & $(1,7)$ & $(1,8)$ & $(1,9)$ & $(1,10)$ & $(1,11)$ & $(1,12)$ & $(1,13)$ & $(1,14)$ & $(1,15)$ & $(1,16)$ \\
\hline & 1 & 2 & $(2,1)$ & $(2,2)$ & $(2,3)$ & $(2,4)$ & $(2,5)$ & $(2,6)$ & $(2,7)$ & $(2,8)$ & $(2,9)$ & $(2,10)$ & $(2,11)$ & $(2,12)$ & $(2,13)$ & $(2,14)$ & $(2,15)$ & $(2,16)$ \\
\hline \multirow{6}{*}{$\begin{array}{l}\text { Shipboard } \\
\text { Service } \\
\text { Converter } \\
\text { Module }\end{array}$} & 1 & 8 & $(3,1)$ & $(3,2)$ & $(3,3)$ & $(3,4)$ & $(3,5)$ & $(3,6)$ & $(3,7)$ & $(3,8)$ & $(3,9)$ & $(3,10)$ & $(3,11)$ & $(3,12)$ & $(3,13)$ & $(3,14)$ & $(3,15)$ & $(3,16)$ \\
\hline & 2 & 7 & $(4,1)$ & $(4,2)$ & $(4,3)$ & $(4,4)$ & $(4,5)$ & $(4,6)$ & $(4,7)$ & $(4,8)$ & $(4,9)$ & $(4,10)$ & $(4,11)$ & $(4,12)$ & $(4,13)$ & $(4,14)$ & $(4,15)$ & $(4,16)$ \\
\hline & 3 & 6 & $(5,1)$ & $(5,2)$ & $(5,3)$ & $(5,4)$ & $(5,5)$ & $(5,6)$ & $(5,7)$ & $(5,8)$ & $(5,9)$ & $(5,10)$ & $(5,11)$ & $(5,12)$ & $(5,13)$ & $(5,14)$ & $(5,15)$ & $(5,16)$ \\
\hline & 4 & 5 & $\begin{array}{l}(6,1) \\
\end{array}$ & $\begin{array}{l}(6,2) \\
\end{array}$ & $\begin{array}{l}(6,3) \\
\end{array}$ & $\begin{array}{l}(6,4) \\
\end{array}$ & $\begin{array}{l}(6,5) \\
\end{array}$ & $\begin{array}{l}(6,6) \\
\end{array}$ & $(6,7)$ & $(6,8)$ & $(6,9)$ & $(6,10)$ & $(6,11)$ & $(6,12)$ & $(6,13)$ & $(6,14)$ & $(6,15)$ & $(6,16)$ \\
\hline & 5 & 4 & $(7,1)$ & $(7,2)$ & $(7,3)$ & $(7,4)$ & $(7,5)$ & $(7,6)$ & $(7,7)$ & $(7,8)$ & $(7,9)$ & $(7,10)$ & $(7,11)$ & $(7,12)$ & $(7,13)$ & $(7,14)$ & $(7,15)$ & $(7,16)$ \\
\hline & 6 & 3 & $(8,1)$ & $(8,2)$ & $(8,3)$ & $(8,4)$ & $(8,5)$ & $(8,6)$ & $(8,7)$ & $(8,8)$ & $(8,9)$ & $(8,10)$ & $(8,11)$ & $(8,12)$ & $(8,13)$ & $(8,14)$ & $(8,15)$ & $(8,16)$ \\
\hline \multirow{5}{*}{$\begin{array}{l}\text { Constant } \\
\text { Power Load }\end{array}$} & 1 & 13 & $(9,1)$ & $(9,2)$ & $(9,3)$ & $(9,4)$ & $(9,5)$ & $(9,6)$ & $(9,7)$ & $(9,8)$ & $(9,9)$ & $(9,10)$ & $(9,11)$ & $(9,12)$ & $(9,13)$ & $(9,14)$ & $(9,15)$ & $(9,16)$ \\
\hline & 2 & 12 & $(10,1)$ & $(10,2)$ & $(10,3)$ & $(10,4)$ & $(10,5)$ & $(10,6)$ & $(10,7)$ & $(10,8)$ & $(10,9)$ & $(10,10)$ & $(10,11)$ & $(10,12)$ & $(10,13)$ & $(10,14)$ & $(10,15)$ & $(10,16)$ \\
\hline & 3 & 11 & $(11,1)$ & $(11,2)$ & $(11,3)$ & $(11,4)$ & $(11,5)$ & $(11,6)$ & $(11,7)$ & $(11,8)$ & $(11,9)$ & $(11,10)$ & $(11,11)$ & $(11,12)$ & $(11,13)$ & $(11,14)$ & $(11,15)$ & $(11,16)$ \\
\hline & 4 & 10 & $(12,1)$ & $(12,2)$ & $(12,3)$ & $(12,4)$ & $(12,5)$ & $(12,6)$ & $(12,7)$ & $(12,8)$ & $(12,9)$ & $(12,10)$ & $(12,11)$ & $(12,12)$ & $(12,13)$ & $(12,14)$ & $(12,15)$ & $(12,16)$ \\
\hline & 5 & 9 & $(13,1)$ & $(13,2)$ & $(13,3)$ & $(13,4)$ & $(13,5)$ & $(13,6)$ & $(13,7)$ & $(13,8)$ & $(13,9)$ & $(13,10)$ & $(13,11)$ & $(13,12)$ & $(13,13)$ & $(13,14)$ & $(13,15)$ & $(13,16)$ \\
\hline Pulse Load & 1 & 14 & $(14,1)$ & $(14,2)$ & $(14,3)$ & $(14,4)$ & $(14,5)$ & $(14,6)$ & $(14,7)$ & $(14,8)$ & $(14,9)$ & $(14,10)$ & $(14,11)$ & $(14,12)$ & $(14,13)$ & $(14,14)$ & $(14,15)$ & $(14,16)$ \\
\hline Motor Control & 1 & 15 & $(15,1)$ & $(15,2)$ & $(15,3)$ & $(15,4)$ & $(15,5)$ & $(15,6)$ & $(15,7)$ & $(15,8)$ & $(15,9)$ & $(15,10)$ & $(15,11)$ & $(15,12)$ & $(15,13)$ & $(15,14)$ & $(15,15)$ & $(15,16)$ \\
\hline \multirow{2}{*}{ Propulsion Drive } & 1 & 17 & $(17,1)$ & $(17,2)$ & $(17,3)$ & $(17,4)$ & $(17,5)$ & $(17,6)$ & $(17,7)$ & $(17,8)$ & $(17,9)$ & $(17,10)$ & $(17,11)$ & $(17,12)$ & $(17,13)$ & $(17,14)$ & $(17,15)$ & $(17,16)$ \\
\hline & 2 & 16 & $(16,1)$ & $(16,2)$ & $(16,3)$ & $(16,4)$ & $(16,5)$ & $(16,6)$ & $(16,7)$ & $(16,8)$ & $(16,9)$ & $(16,10)$ & $(16,11)$ & $(16,12)$ & $(16,13)$ & $(16,14)$ & $(16,15)$ & $(16,16)$ \\
\hline \multirow{2}{*}{ Input Sensor } & 1 & 19 & $(19,1)$ & $(19,2)$ & $(19,3)$ & $(19,4)$ & $(19,5)$ & $(19,6)$ & $(19,7)$ & $(19,8)$ & $(19,9)$ & $(19,10)$ & $(19,11)$ & $(19,12)$ & $(19,13)$ & $(19,14)$ & $(19,15)$ & $(19,16)$ \\
\hline & 2 & 18 & $(18,1)$ & $(18,2)$ & $(18,3)$ & $(18,4)$ & $(18,5)$ & $(18,6)$ & $(18,7)$ & $(18,8)$ & $(18,9)$ & $(18,10)$ & $(18,11)$ & $(18,12)$ & $(18,13)$ & $(18,14)$ & $(18,15)$ & $(18,16)$ \\
\hline \multirow{8}{*}{ Relay } & 1 & 8.1 & $(20,1)$ & $(20,2)$ & $(20,3)$ & $(20,4)$ & $(20,5)$ & $(20,6)$ & $(20,7)$ & $(20,8)$ & $(20,9)$ & $(20,10)$ & $(20,11)$ & $(20,12)$ & $(20,13)$ & $(20,14)$ & $(20,15)$ & $(20,16)$ \\
\hline & 2 & 7.1 & $(21,1)$ & $(21,2)$ & $(21,3)$ & $(21,4)$ & $(21,5)$ & $(21,6)$ & $(21,7)$ & $(21,8)$ & $(21,9)$ & $(21,10)$ & $(21,11)$ & $(21,12)$ & $(21,13)$ & $(21,14)$ & $(21,15)$ & $(21,16)$ \\
\hline & 3 & 6.3 & $(22,1)$ & $(22,2)$ & $(22,3)$ & $(22,4)$ & $(22,5)$ & $(22,6)$ & $(22,7)$ & $(22,8)$ & $(22,9)$ & $(22,10)$ & $(22,11)$ & $(22,12)$ & $(22,13)$ & $(22,14)$ & $(22,15)$ & $(22,16)$ \\
\hline & 4 & 5.3 & $(23,1)$ & $(23,2)$ & $(23,3)$ & $(23,4)$ & $(23,5)$ & $(23,6)$ & $(23,7)$ & $(23,8)$ & $(23,9)$ & $(23,10)$ & $(23,11)$ & $(23,12)$ & $(23,13)$ & $(23,14)$ & $(23,15)$ & $(23,16)$ \\
\hline & 5 & 6.1 & $(24,1)$ & $(24,2)$ & $(24,3)$ & $(24,4)$ & $(24,5)$ & $(24,6)$ & $(24,7)$ & $(24,8)$ & $(24,9)$ & $(24,10)$ & $(24,11)$ & $(24,12)$ & $(24,13)$ & $(24,14)$ & $(24,15)$ & $(24,16)$ \\
\hline & 6 & 5.1 & $(25,1)$ & $(25,2)$ & $(25,3)$ & $(25,4)$ & $(25,5)$ & $(25,6)$ & $(25,7)$ & $(25,8)$ & $(25,9)$ & $(25,10)$ & $(25,11)$ & $(25,12)$ & $(25,13)$ & $(25,14)$ & $(25,15)$ & $(25,16)$ \\
\hline & 7 & 4.1 & $(26,1)$ & $(26,2)$ & $(26,3)$ & $(26,4)$ & $(26,5)$ & $(26,6)$ & $(26,7)$ & $(26,8)$ & $(26,9)$ & $(26,10)$ & $(26,11)$ & $(26,12)$ & $(26,13)$ & $(26,14)$ & $(26,15)$ & $(26,16)$ \\
\hline & 8 & 3.1 & $(27,1)$ & $(27,2)$ & $(27,3)$ & $(27,4)$ & $(27,5)$ & $(27,6)$ & $(27,7)$ & $(27,8)$ & $(27,9)$ & $(27,10)$ & $(27,11)$ & $(27,12)$ & $(27,13)$ & $(27,14)$ & $(27,15)$ & $(27,16)$ \\
\hline
\end{tabular}




\subsubsection{GUI User Guide}

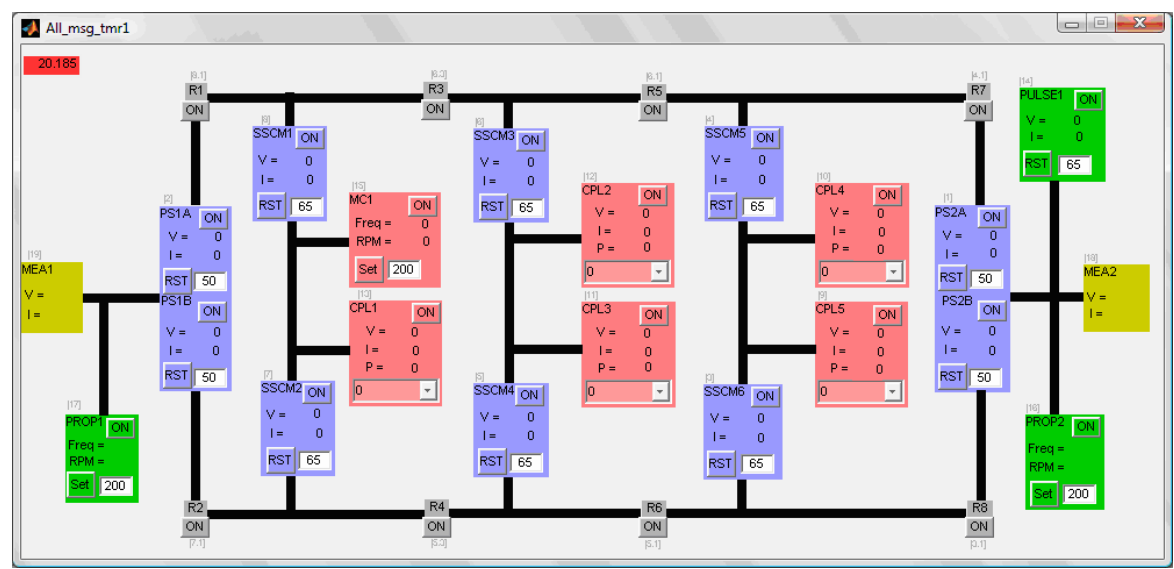

Figure 44: Timer is Turned On.

A right click on the "Timer Indicator" at the left-top corner of the GUI starts the initialization function for periodic CAN messaging and the timer. A blinking red indicator shown in Figure 44 stands for an active timer. All the display areas on the GUI are loaded with the values read from the devices. Figure 45 shows the GUI screen after the power supply PS1A is turned on. A value 50 is selected as the default set-point for PS1A. The display settles at 50 after an overshoot. Value 50 corresponds to 35 volts using the relation shown in equation (6). The value of $R$ in this case is $18 \mathrm{k}$.

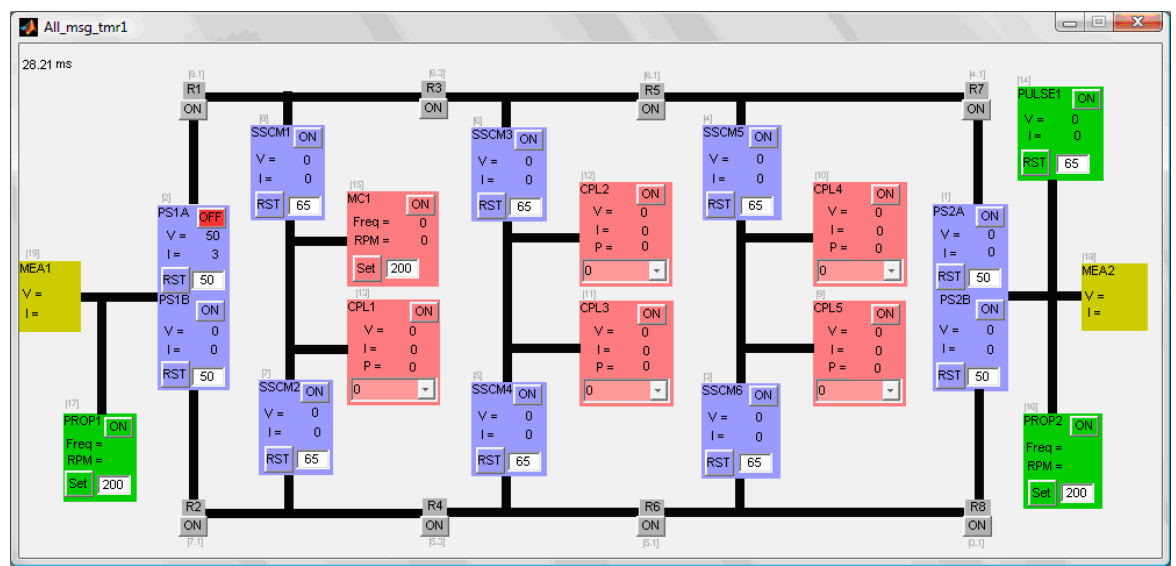

Figure 45: PS1 Turn On

Figure 46 shows the GUI after breaker R1 and SSCM1 are turned on. The value of $R$ in this case is $12 \mathrm{k}$ and the value 65 stands for 30 volts using equation (6). 


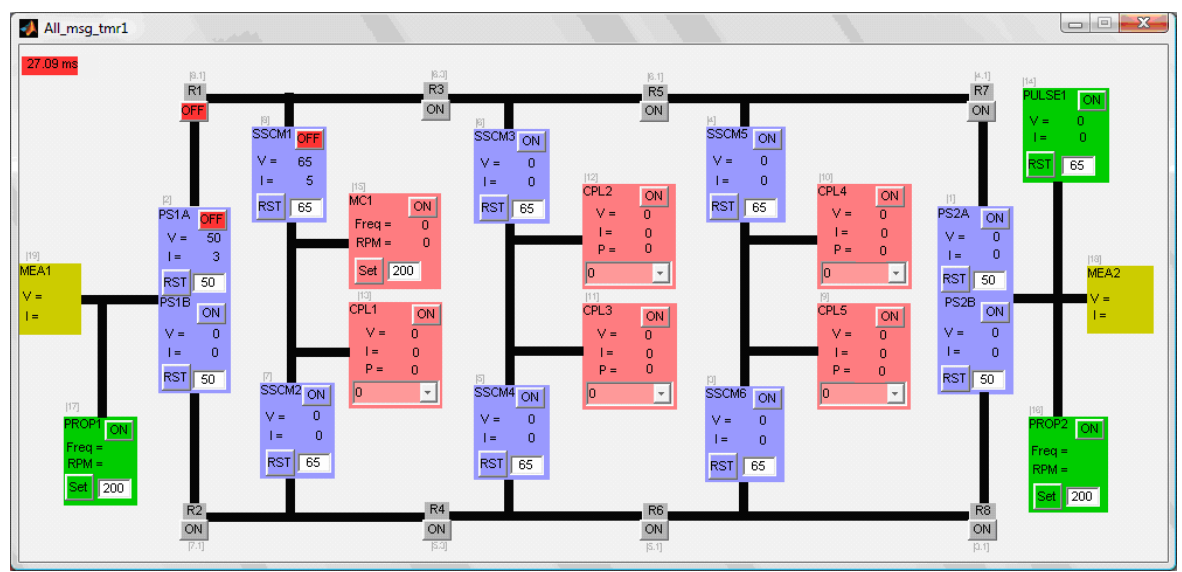

Figure 46: PS1, Breaker R1, and SSCM1 are Turned On.

Figure 47 shows the pull down combo box for constant power load control. Also, the SSCM5 voltage set-point's value is set to 75 , which corresponds to 35 volts using equation (6).

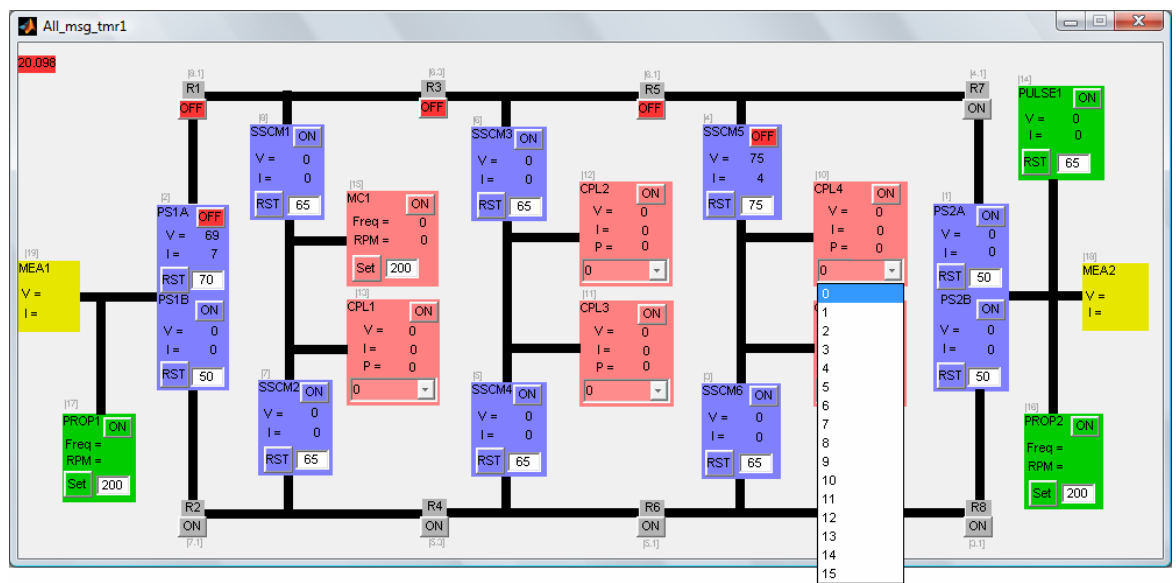

Figure 47: GUI Interface for Constant Power Load.

Figure 48 below shows the main timer function. This function updates values displayed on the GUI screen with the values received at the CAN interface periodically. The function uses switch statements to move data to appropriate text fields. Values like from device (fd) and data designator ( $\mathrm{dd}$ ) are checked for this purpose. A while loop is used to continuously read and empty the PCAN USB buffer. The PCAN USB has been described in section 2.2.5.

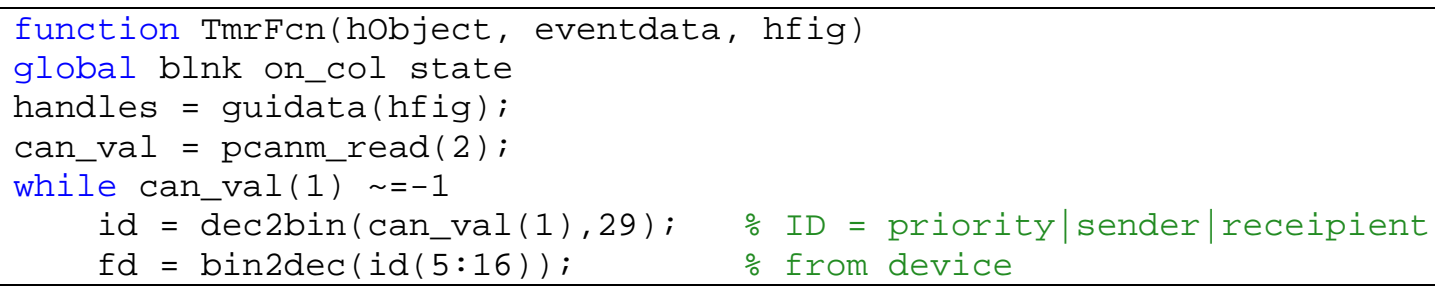




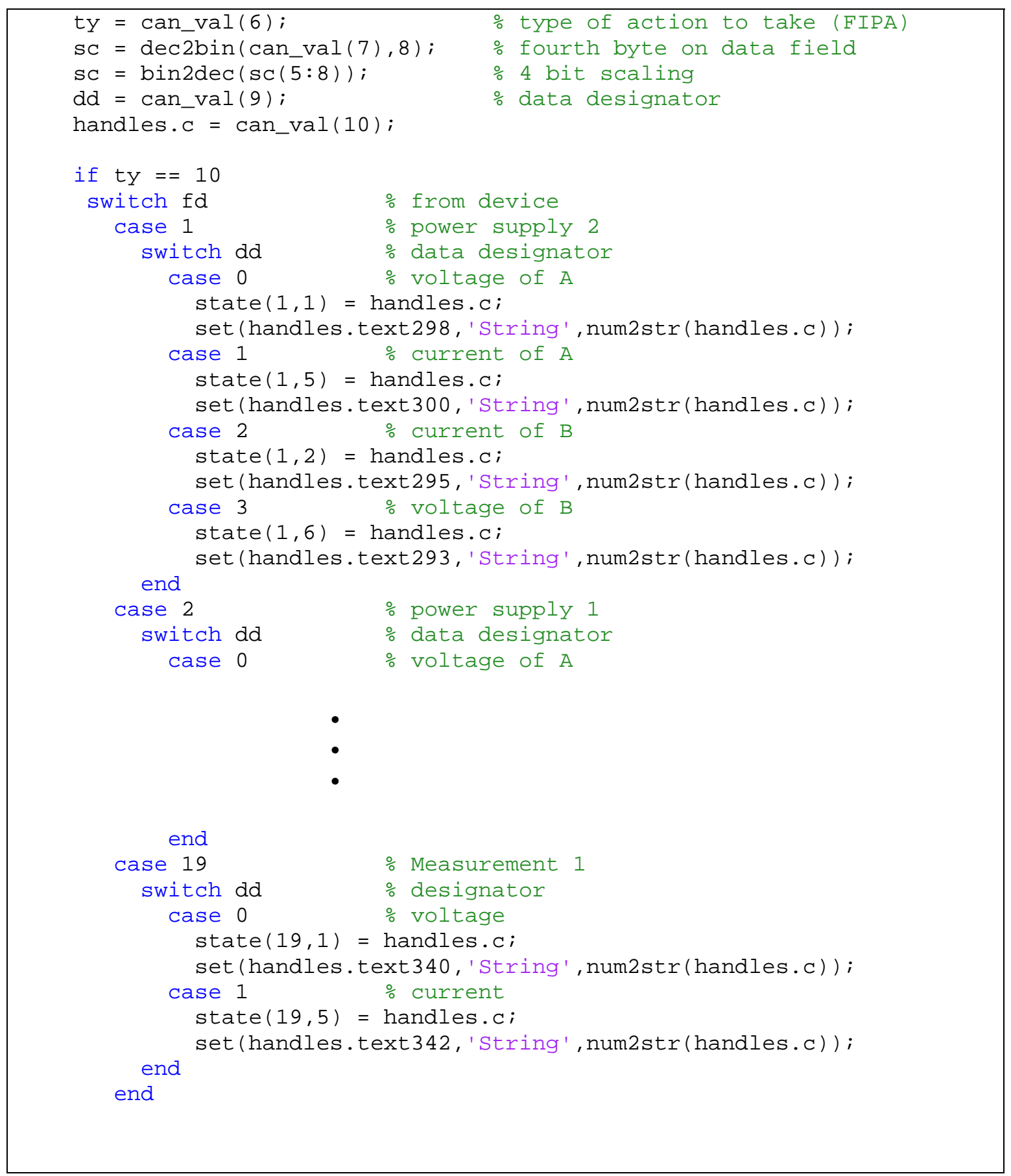

Figure 48: Timer Function for Displaying Values

\subsection{Testbed Construction}

The testbed was built on an OBS board of size $4.5^{\prime}$ x $2.5^{\prime}$, and all devices have been marked by color codes as shown in Figure 49. 


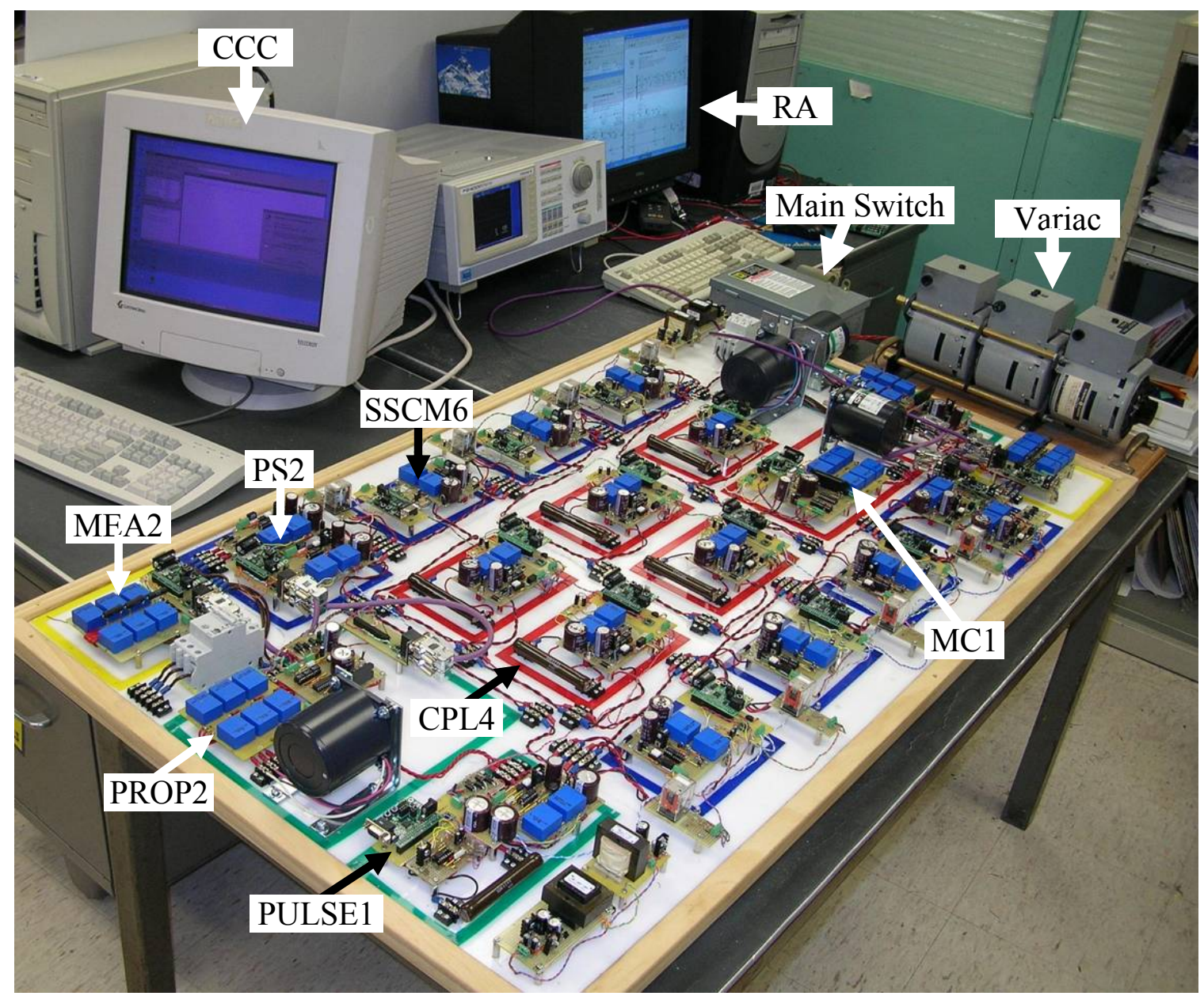

Figure 49: Completely Assembled Testbed Integrated with RA and CCC. 


\section{Chapter 4 Test and Results}

Individual components on the testbed have been tested for steady-state and transient load change responses. The test data includes the voltage and current profiles of inputs and outputs for the converters and loads. Testing also includes performance of safety features like overload and short circuit cutoff. Testing was also performed to check the converter startup currents and voltage profiles. The motor drive was tested at different frequencies.

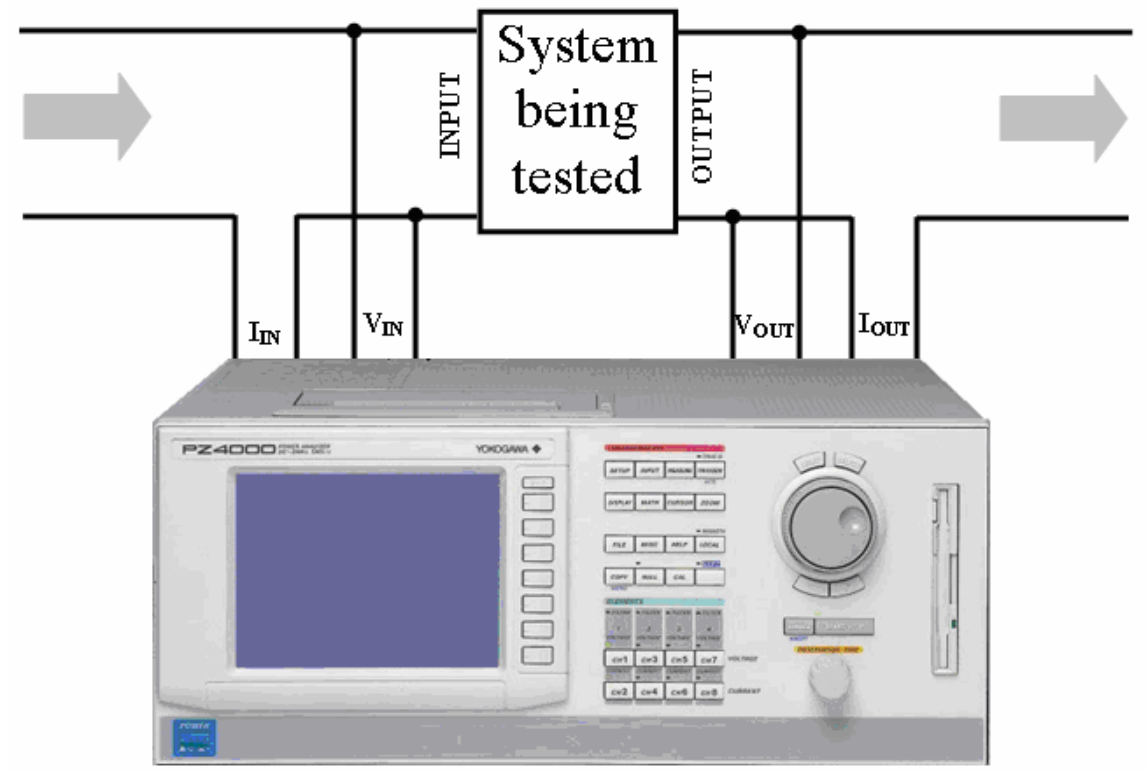

Figure 50: Test Setup Using Power Analyzer PZ4000

Electric power was supplied from a 3-phase, non-isolated variac connected to the $\mathrm{AC}$ mains. Yokogawa Power Analyzer PZ4000 was used for the measurement of voltages and currents. The input current, input voltage, output current, and output voltage were logged. The power analyzer allows screenshots to be saved and these have been documented in this chapter along with an explanation on the test conditions and results.

A 3-phase disconnect switch and two Miniature Circuit Breakers (MCB) have been added to the testbed to ensure protection as shown in Figure 51: . 


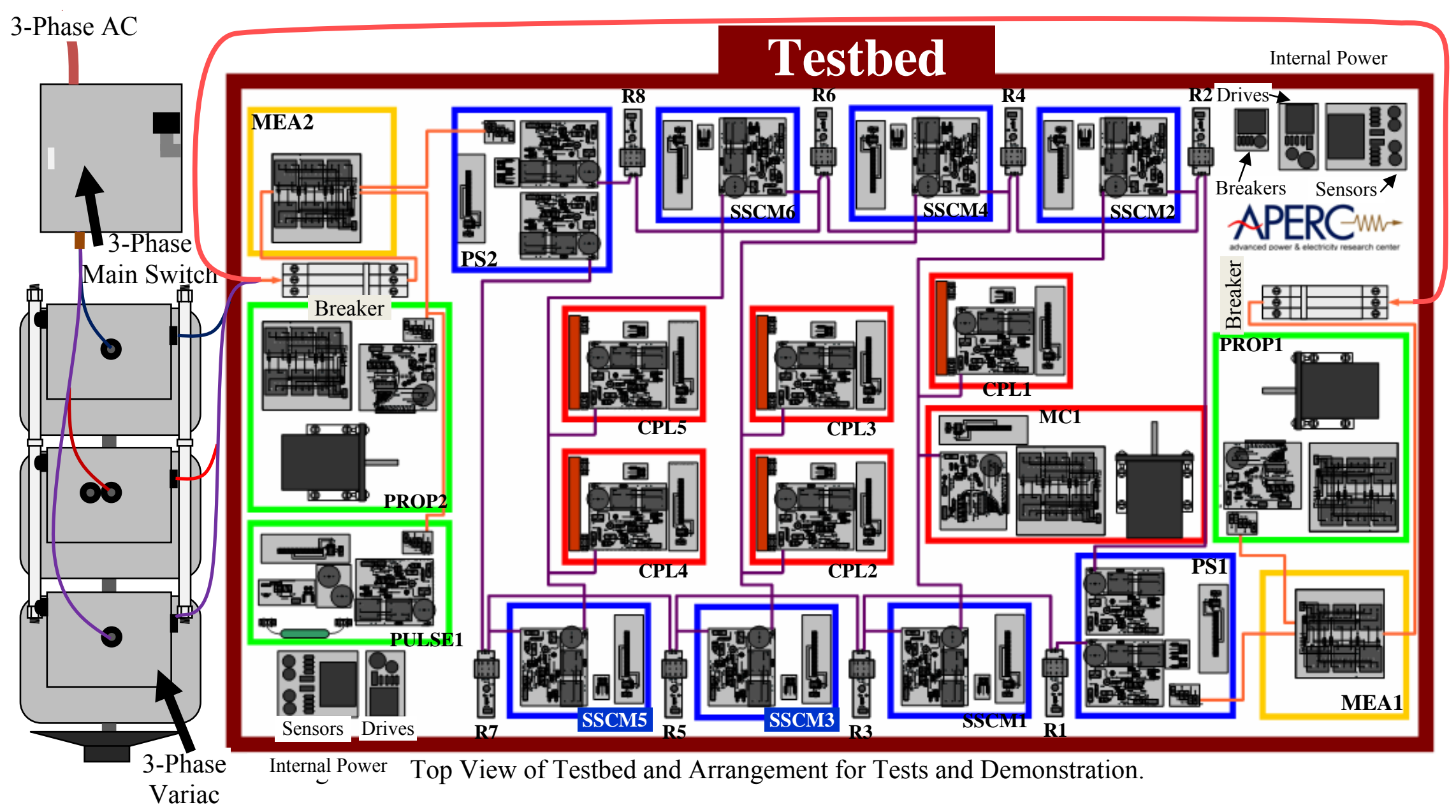




\subsection{Power Supply}

Figure 52 shows the startup of the power supply and measurements taken after the voltage settles. Settling time for the voltage is about $220 \mathrm{~ms}$. Ripple in the input current is due to the peak charging of input capacitor through rectifier diodes. Considering the sampling rate of $10 \mathrm{~ms}$ for all the blocks in the controller model, this settling time is less than 22 samples away from the startup point. The voltage set-point was programmed to be 43 volts (60 in the GUI).

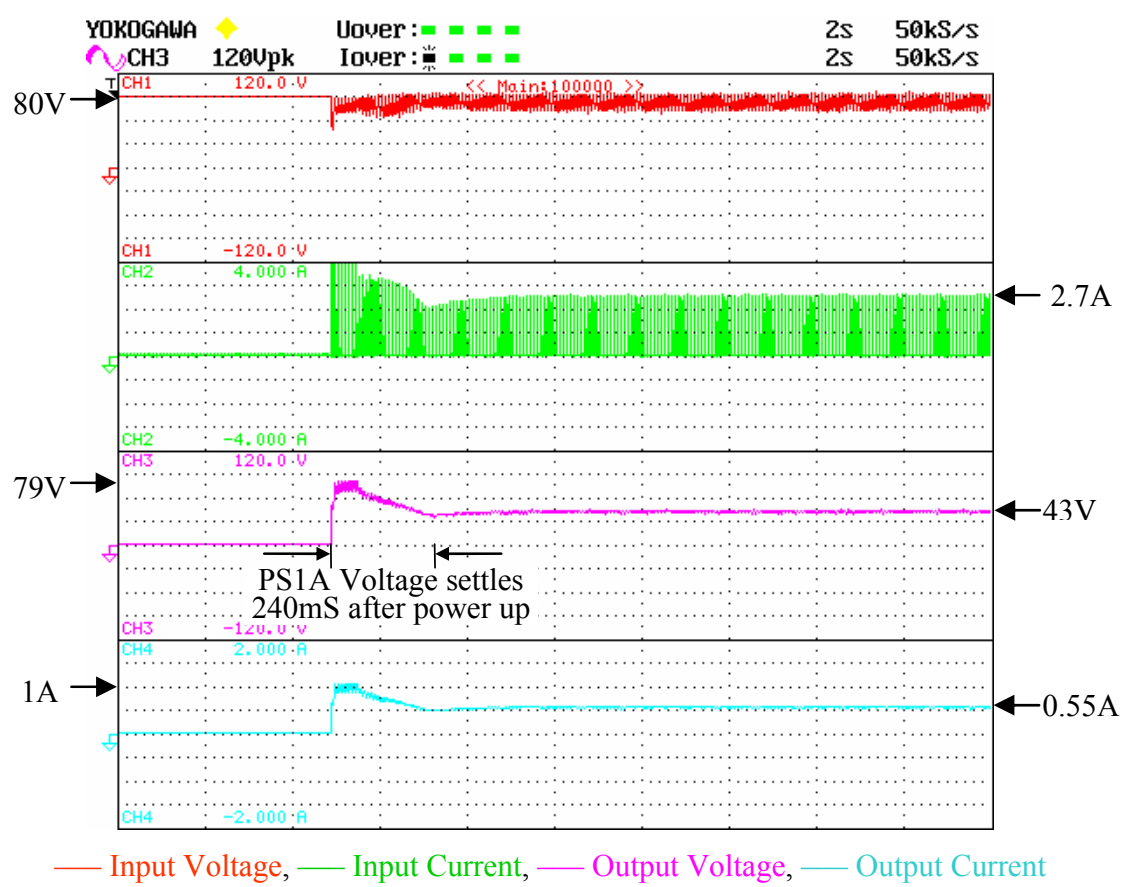

Figure 52: Power Supply Startup

Figure 53 shows a step increase in load. Load current was increased from $0.18 \mathrm{~A}$ to 0.68 A. This increase of $0.5 \mathrm{~A}$ caused the voltage to dip down to $30 \mathrm{~V}$ before the controller responded. It also shows the settling time of $300 \mathrm{~ms}$ for the controller and the increase in output voltage ripple with the increase in load. 


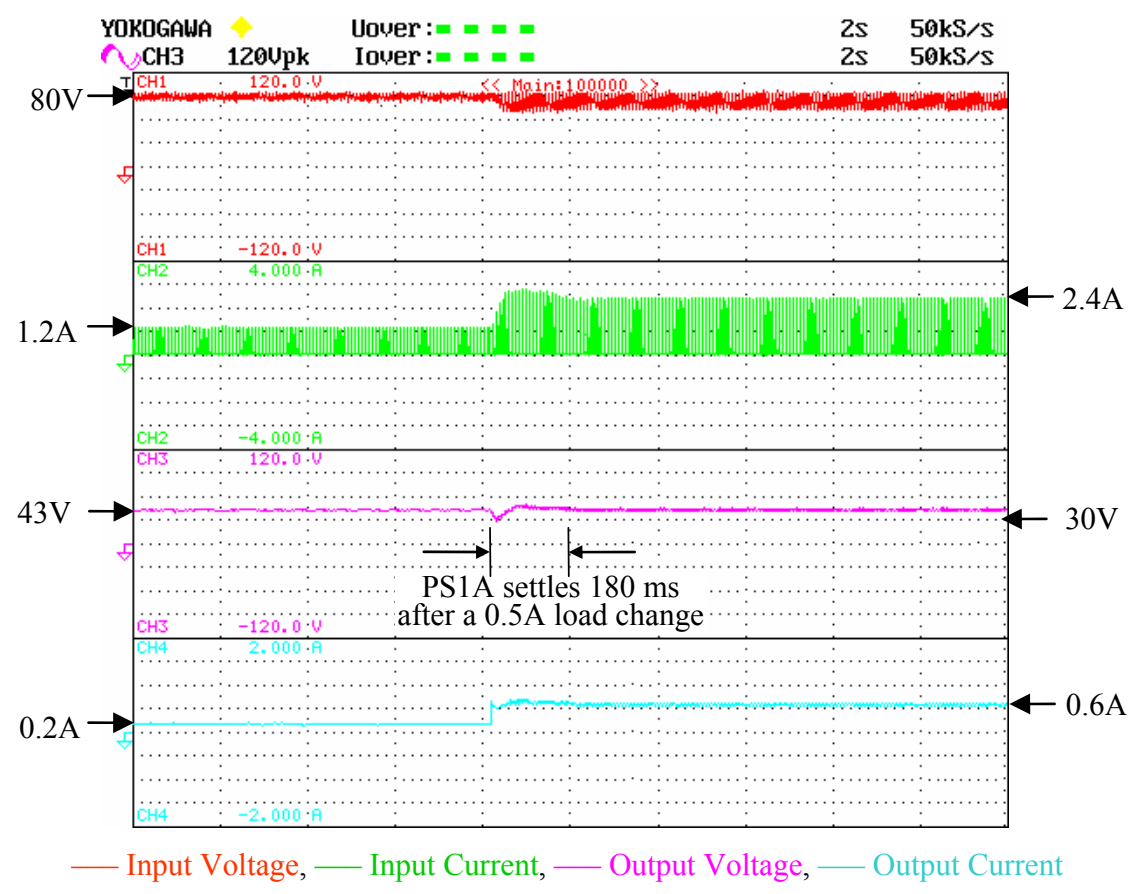

Figure 53: Step load increase

Figure 54 shows the step response for a $0.5 \mathrm{~A}$ decrease load current. A load decrease from 0.63 A to 0.18 A results in a settling time of $240 \mathrm{~ms}$.

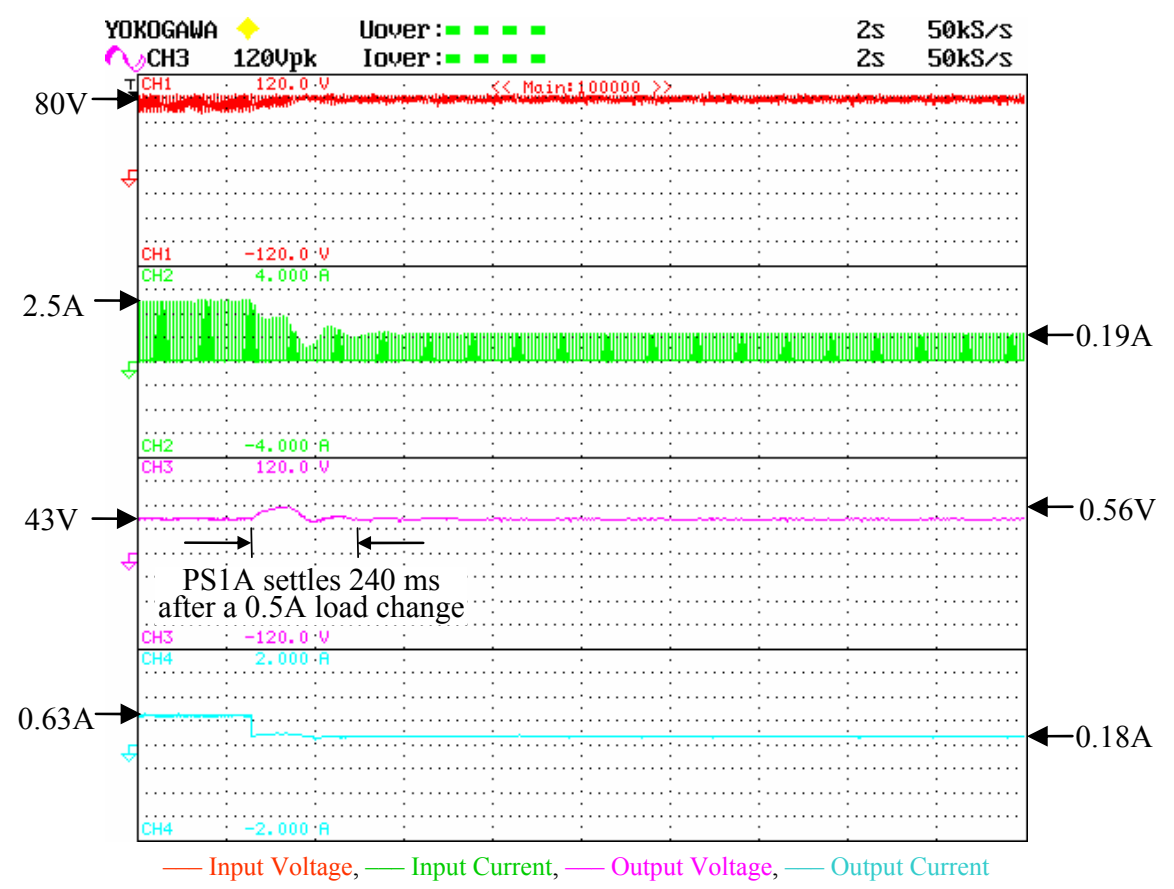

Figure 54: Step load decrease

Overload cut-off is designed to avoid high currents that can occur due to the charging of input capacitors in the succeeding converter. The following figure shows voltage and 
current for an overload. An overload must stay for $160 \mathrm{~ms}$ to trigger an overload shutdown.

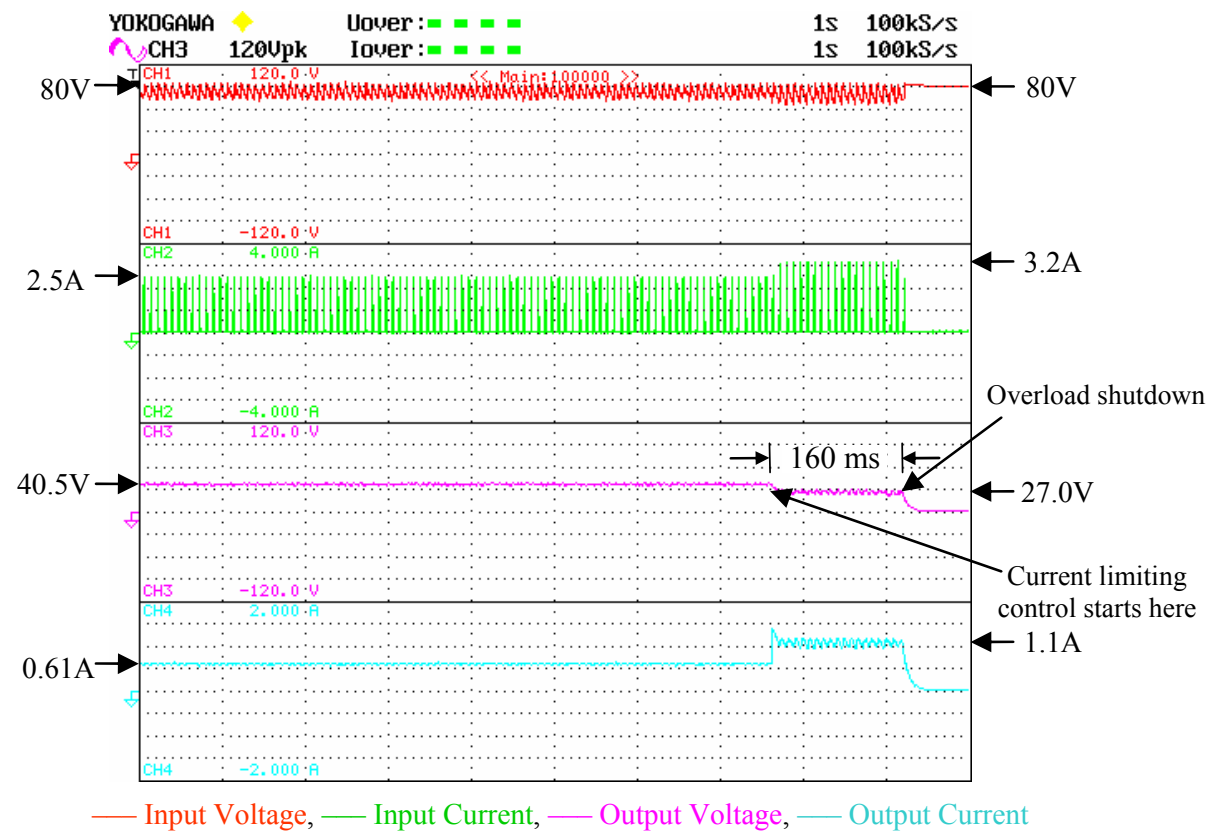

Figure 55: Overload Cutoff

\subsection{Shipboard Service Converter Module}

This converter is connected to the output of the power supply during the testing as it will be during normal operation. Figure 56 shows the startup of the SSCM. The power supply maintains peak voltage at the input capacitor of SSCM. As soon as the SSCM turns on, the power supply output voltage dips down to $32 \mathrm{~V}$ before the controller acts. For this testing, power supply has been set to provide $48 \mathrm{~V}$ output. In approximately $50 \mathrm{~ms}$ of settling time, the power supply maintains the output voltage but as the SSCM converter only sees a high voltage beyond this point it settles in $250 \mathrm{~ms}$. 


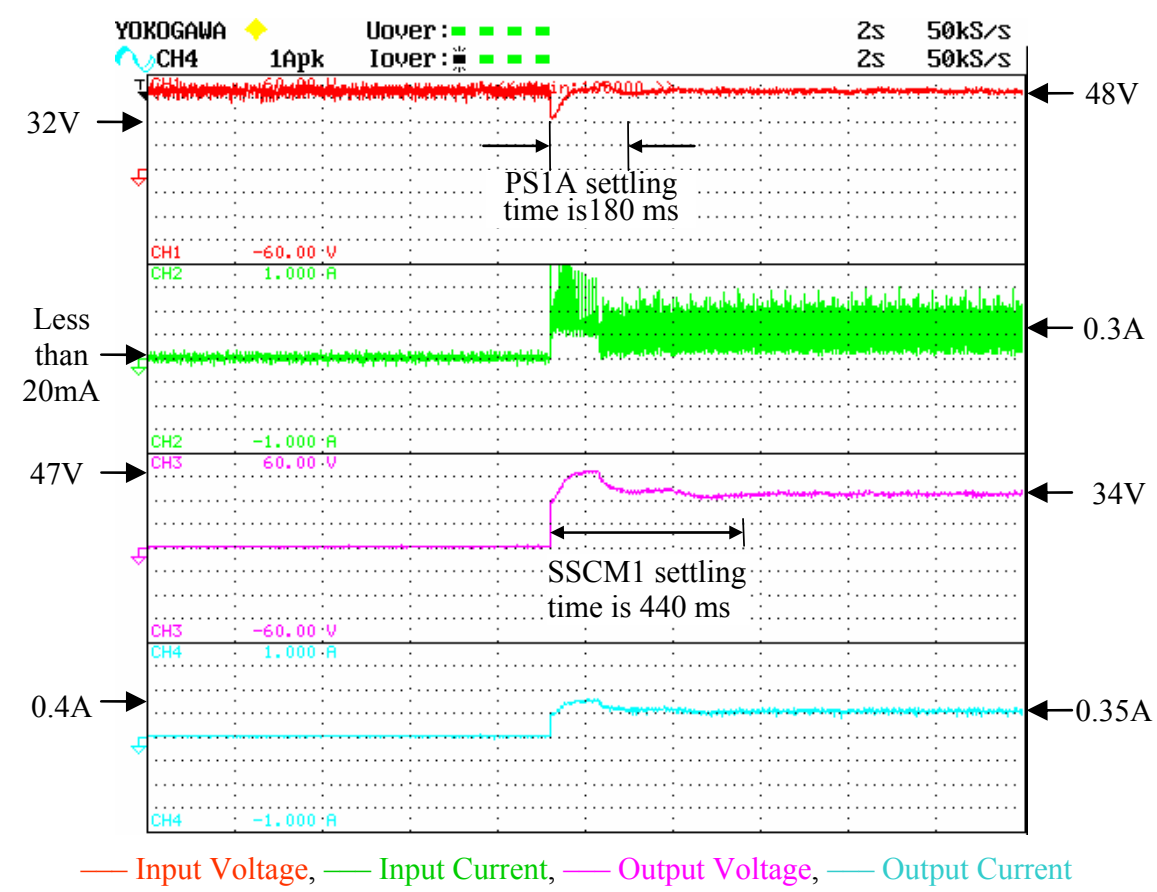

Figure 56: Shipboard Service Converter Module Startup

SSCM1 responds to a change in voltage at the power supply in about $120 \mathrm{~ms}$.

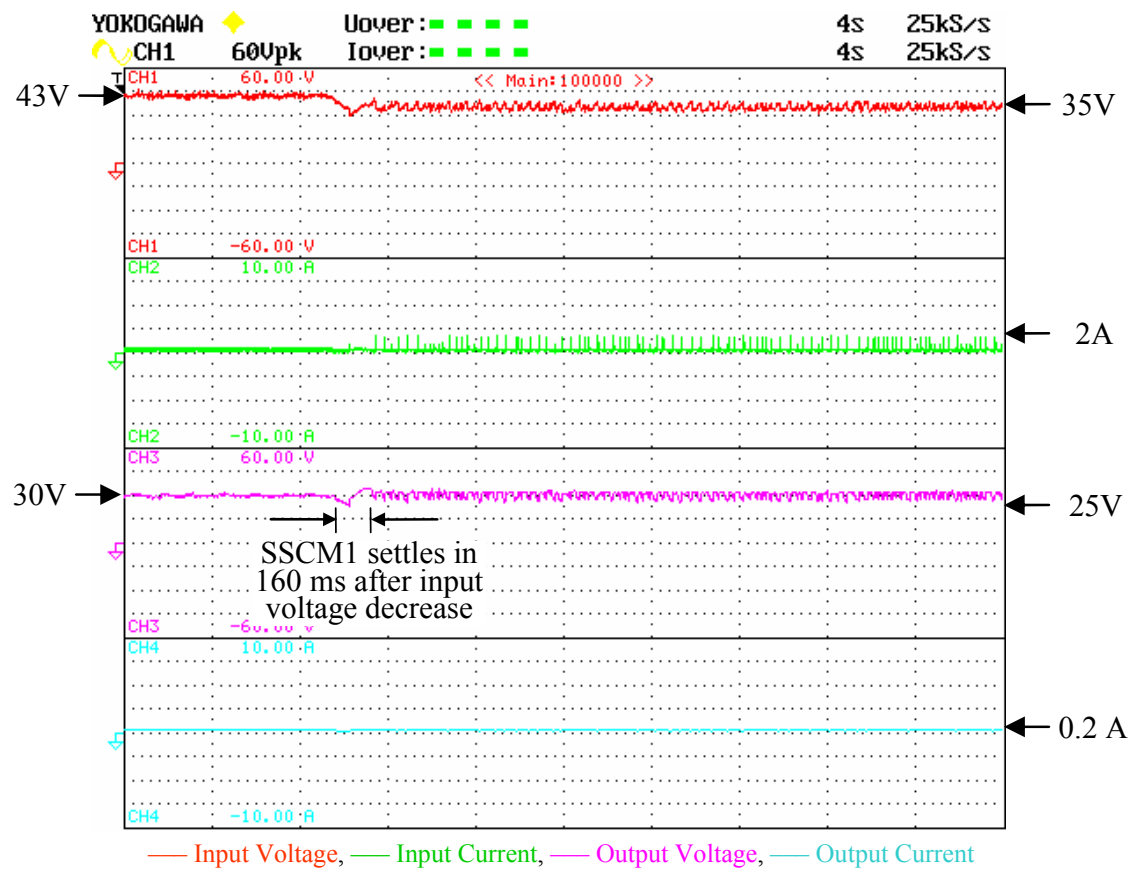

Figure 57: Shipboard Service Converter Module Trun-on

A similar test was performed for an increase in the input voltage of the SSCM1. Voltage was increased from $35 \mathrm{~V}$ to $43 \mathrm{~V}$. 


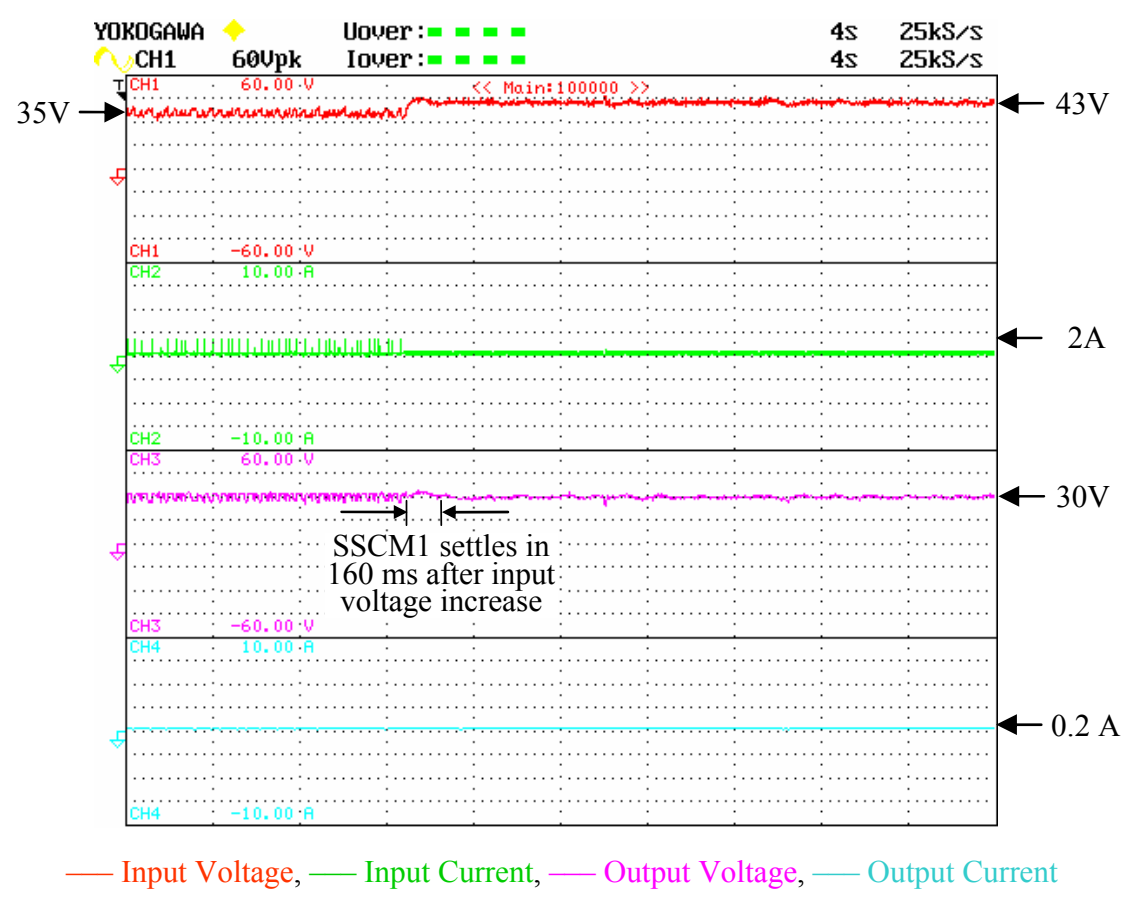

Figure 58: Input voltage increase

\subsection{Pulse Load}

The pulse load was tested in two conditions. The first one evaluates the performance of pulse loading. The second test evaluates the performance of the current control at continuous loading.

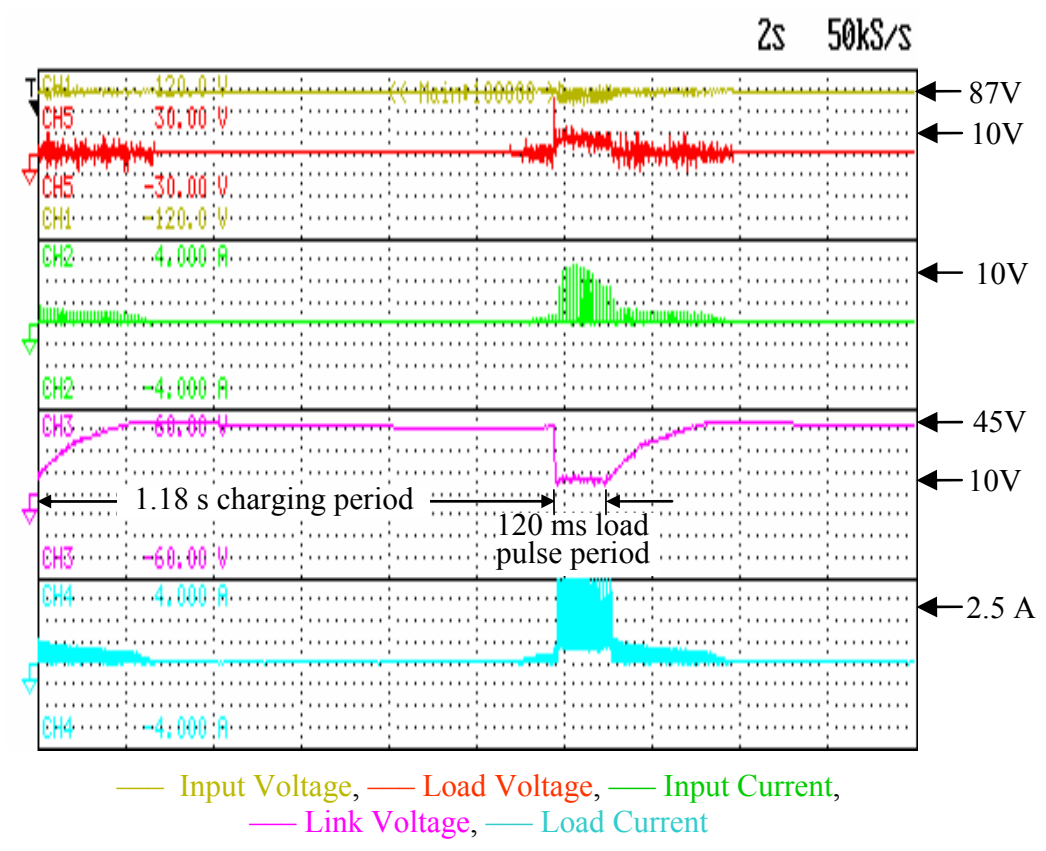

Figure 59: Pulsed Load, Normal Operation. 
The $10 \Omega$ load was turned on for $120 \mathrm{~ms}$ and the capacitor was allowed to charge for 1.18 seconds as shown in Figure 59. The capacitor is charged by a constant current until the voltage reaches the set-point. Afterwards, voltage regulation takes over the current control and maintains the voltage.

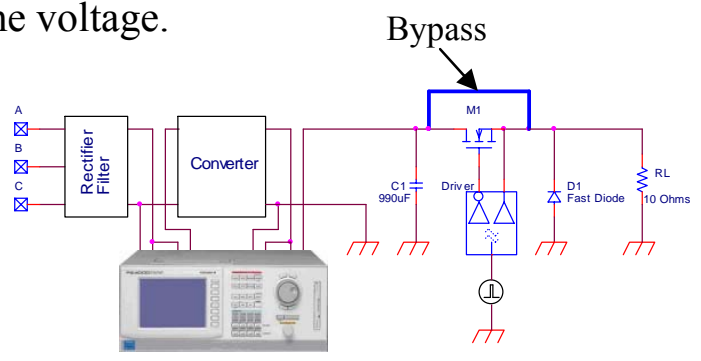

Figure 60: Pulse Load Test Setup.

Second test was intended at evaluating performance of the current regulating controller. The MOSFET for switching output load was bypassed during this testing. Figure 60 shows the setup for the test and Figure 61 shows the results. The bypassing continuously loaded the DC link and forced the converter to stay continuously in the current control mode. A $202 \mathrm{~mA}$ current was measured with a ripple of $87 \mathrm{~mA}$.

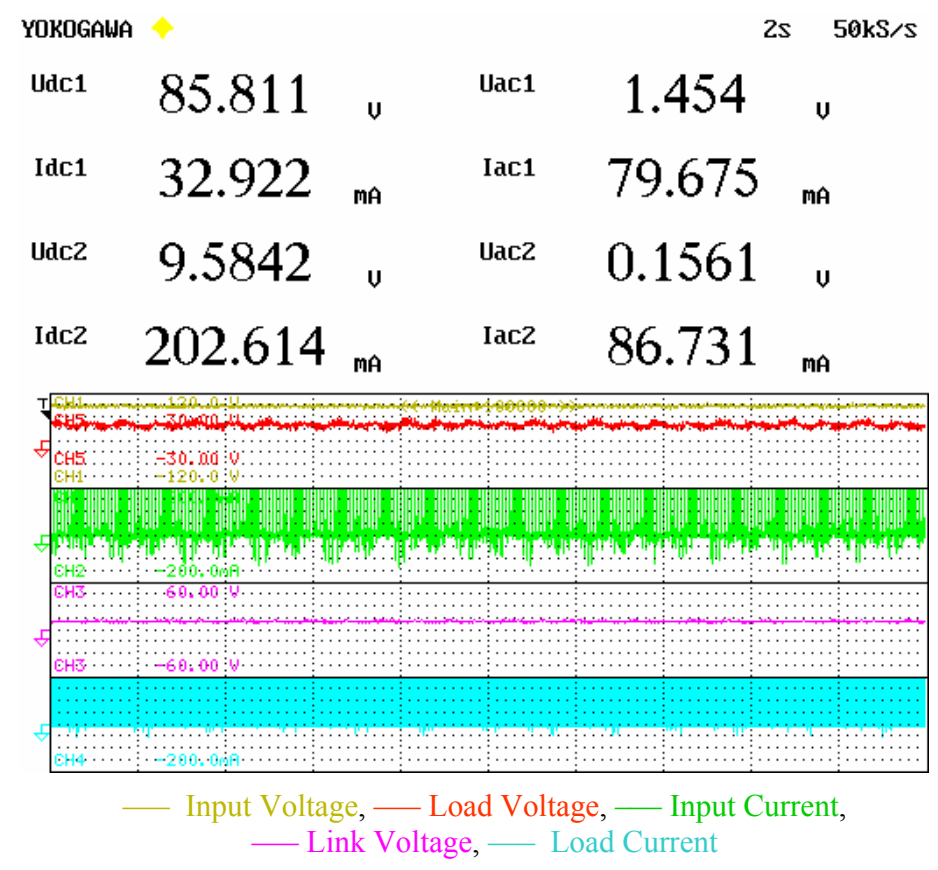

Figure 61: Pulsed Load, Continuous Loading. 


\subsection{Propulsion Drive}

A $60 \mathrm{~Hz}, 3$-phase, 4-pole induction motor was used for this testing. Figure 62 shows the test setup along with the power analyzer.

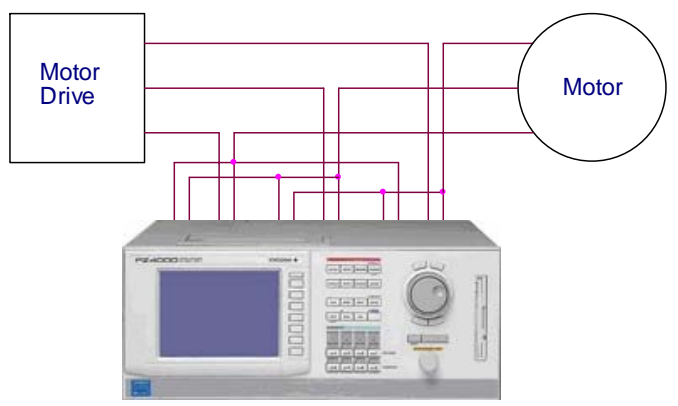

Figure 62: Test Setup for Motor Drive.

The test was performed at two different frequencies. First the lower frequency of $0.27 \mathrm{~Hz}$ (16.2 RPM) was applied. The screenshot of this test in Figure 63 shows a motor speed of 6.5 RPM giving a slip of 1.6 RPM. The line-to-line RMS voltage for the test was fixed at $66 \mathrm{~V}$. An RMS current of $240 \mathrm{~mA}$ was measured for the test.

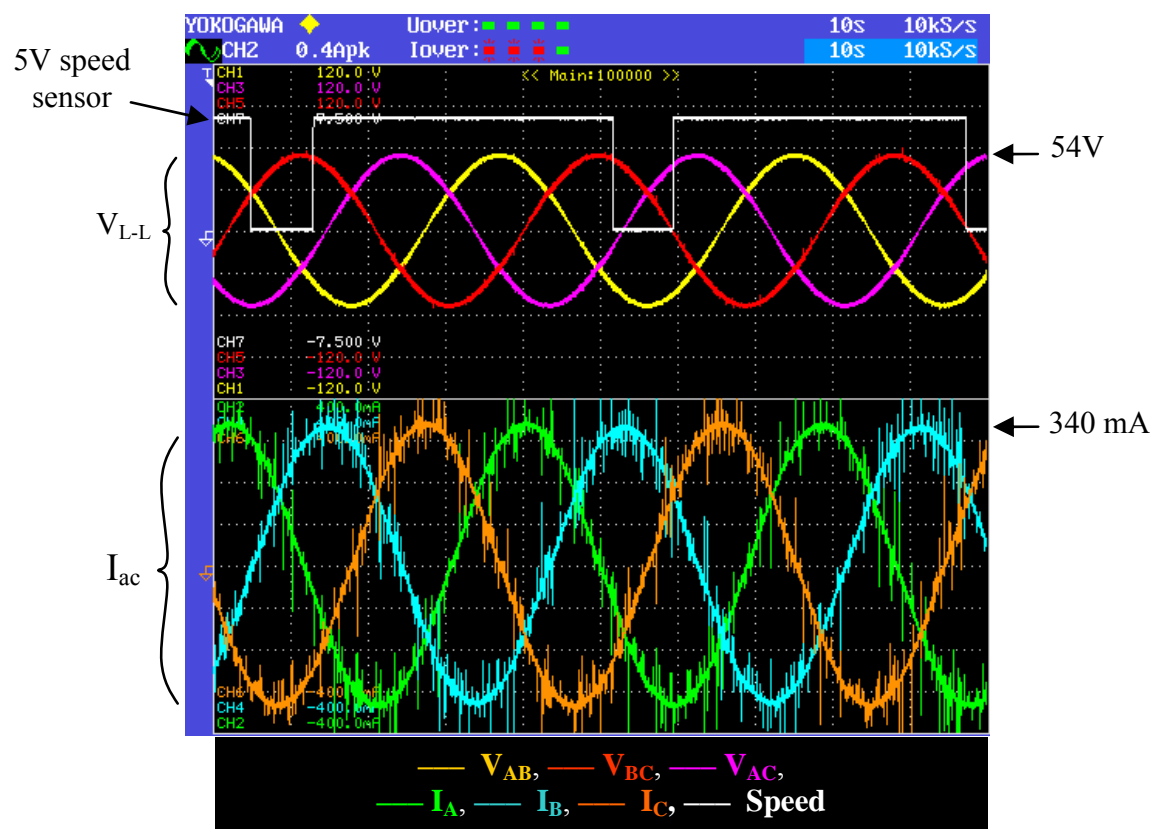

Figure 63: $0.27 \mathrm{~Hz}$ Motor Operation. 
Second test was performed at $71.4 \mathrm{~Hz}$ (4284 RPM) at a line-to-line RMS voltage of $66 \mathrm{~V}$. The measured speed of 1829 RPM showed a slip of 313 RPM. The RMS current per phase was measured $42 \mathrm{~mA}$.

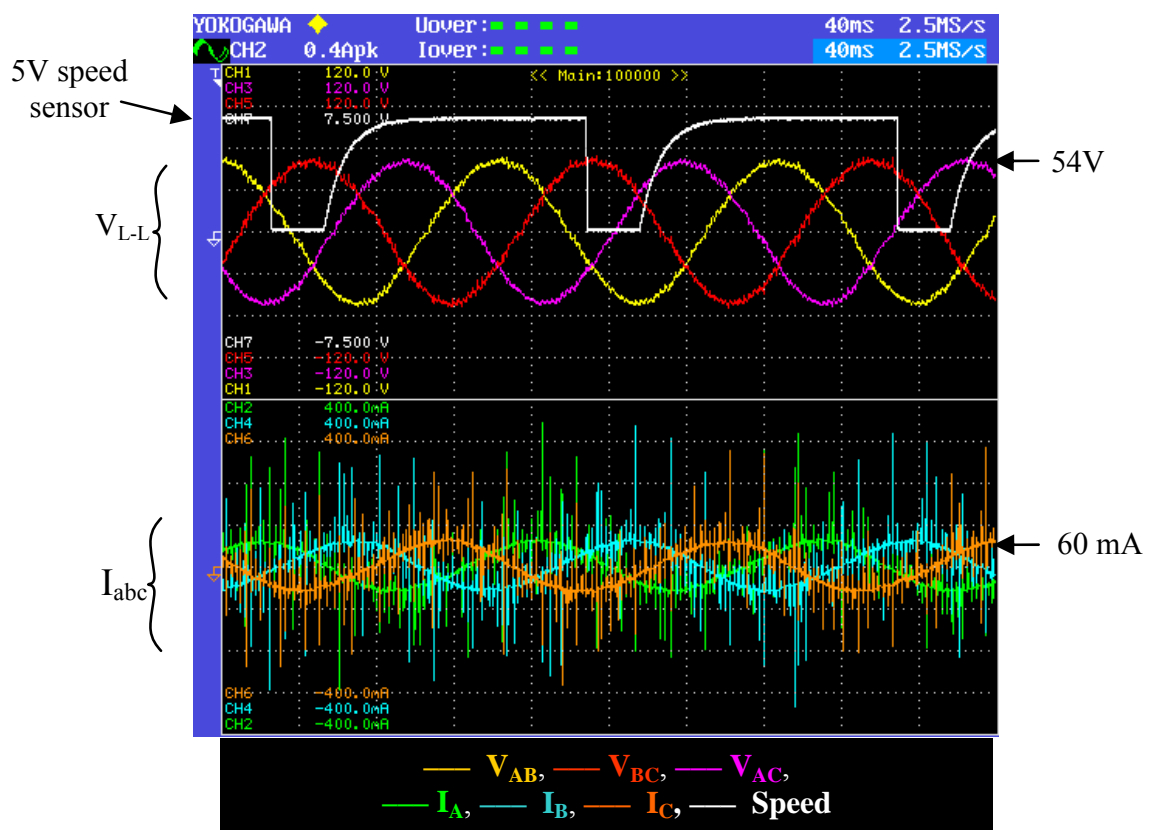

Figure 64: $71 \mathrm{~Hz}$ Motor Operation.

\subsection{Constant Power Load}

The constant power load is designed to sink a fixed amount of power irrespective of the value of the resistor. However, for a given input voltage, resistors can only achieve up to a finite maximum power. Maximum amount of power decreases with increase in load resistance. This controller is designed to provide 16 distinctive power levels at a maximum voltage of $29 \mathrm{~V}$ using a $250 \Omega$ resistor. Integer numbers from 0 to 15 is used as power set points, which has been described in section 3.7. Multiplying this integer number by a factor 0.3 gives the corresponding power in watts. The fourth column in

Table 12 lists the power drawn by CPL. CPL was run at different power levels and the voltages were recorded. 
Table 12 lists the measured voltages at different power levels. The table also lists the calculated voltage for the corresponding voltage levels.

Table 12: Constant Power Load Test with $250 \Omega$

\begin{tabular}{|r|c|c|c|c|c|}
\hline $\begin{array}{c}\text { Power } \\
\text { Level }\end{array}$ & $\begin{array}{c}\text { Calculated } \\
\text { Voltage }\end{array}$ & $\begin{array}{c}\text { Measured } \\
\text { Voltage }\end{array}$ & $\begin{array}{c}\text { Input } \\
\text { Power }\end{array}$ & $\begin{array}{c}\text { Output } \\
\text { Power }\end{array}$ & $\begin{array}{c}\text { Percentage } \\
\text { Efficiency }\end{array}$ \\
\hline 1 & 7.5 & 7.4 & 0.31 & 0.22 & 71.0 \\
\hline 2 & 10.6 & 10.6 & 0.58 & 0.43 & 74.1 \\
\hline 3 & 13.0 & 13.2 & 0.84 & 0.67 & 79.8 \\
\hline 4 & 15.0 & 15.1 & 1.06 & 0.89 & 84.0 \\
\hline 5 & 16.8 & 16.8 & 1.29 & 1.11 & 86.0 \\
\hline 6 & 18.4 & 18.4 & 1.54 & 1.32 & 85.7 \\
\hline 7 & 19.8 & 20.1 & 1.81 & 1.57 & 86.7 \\
\hline 8 & 21.2 & 21.4 & 2.02 & 1.80 & 89.1 \\
\hline 9 & 22.5 & 22.5 & 2.23 & 1.98 & 88.8 \\
\hline 10 & 23.7 & 24.0 & 2.51 & 2.26 & 90.0 \\
\hline 11 & 24.9 & 25.3 & 2.76 & 2.48 & 89.9 \\
\hline 12 & 26.0 & 26.5 & 3.00 & 2.65 & 88.3 \\
\hline 13 & 27.0 & 27.4 & 3.21 & 3.01 & 93.8 \\
\hline 14 & 28.1 & 28.4 & 3.28 & 3.12 & 95.1 \\
\hline 15 & 29.0 & 28.6 & 3.60 & 3.15 & 87.5 \\
\hline
\end{tabular}

Figure 66 shows a plot of the calculated and measured values of voltage across the $250 \Omega$ load resistor. For a given value of load resistor, voltage can be used as the measurement for the power. 


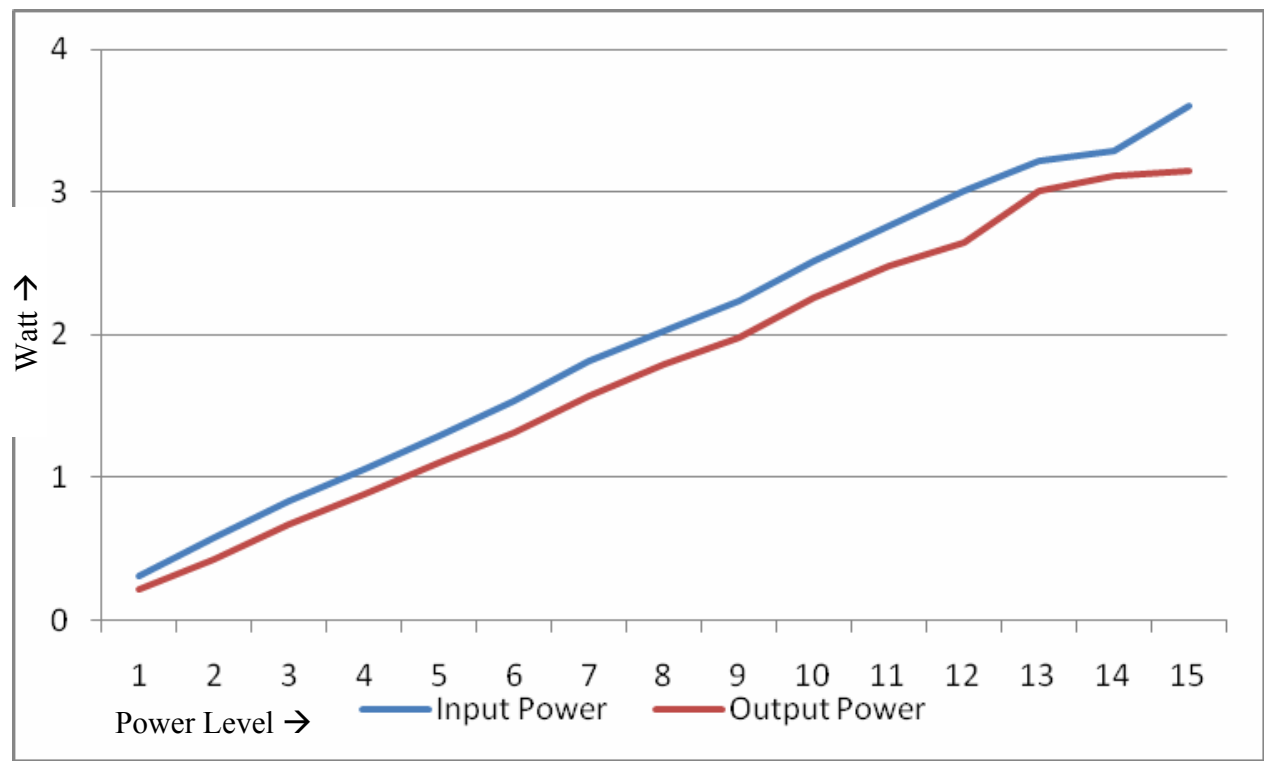

Figure 65: Calculated Voltage Vs Measured Voltage.

The following plot shows efficiency of CPL at different loading levels.

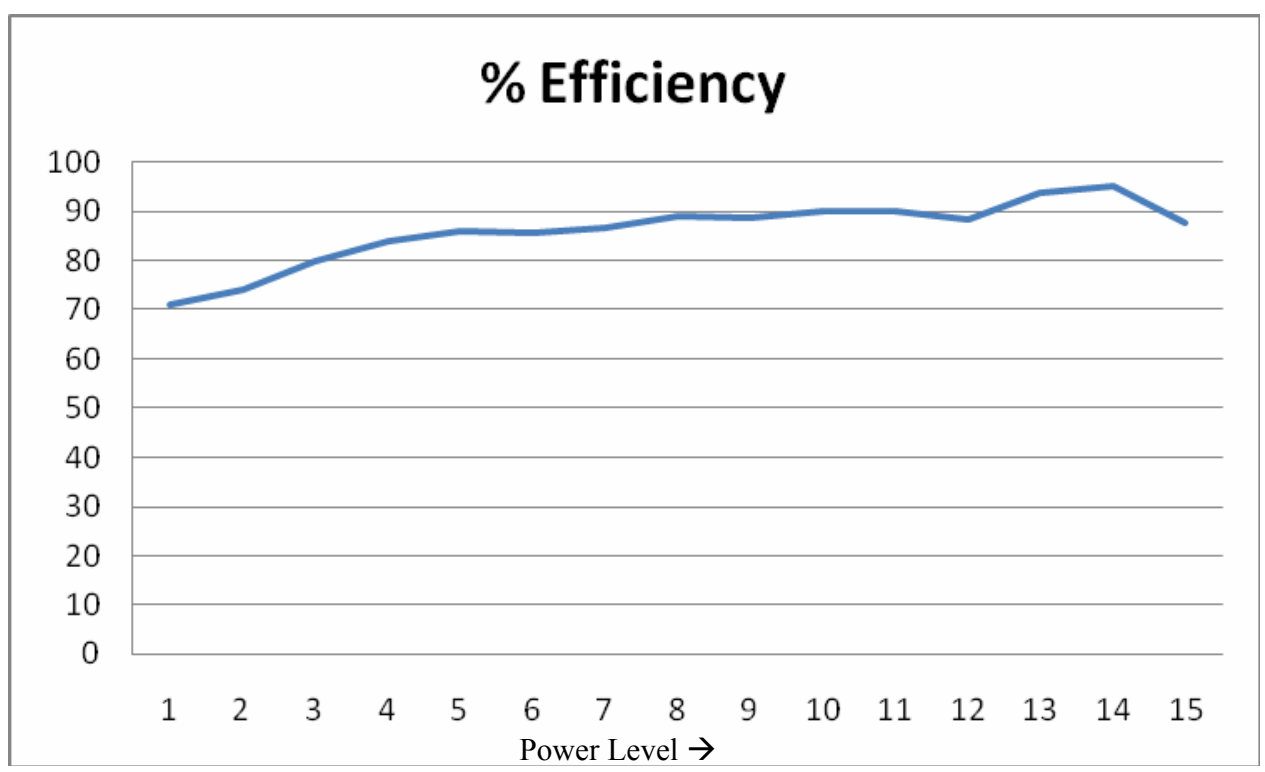

Figure 66: Efficiency at Different Power Levels of CPL.

Figure 67 shows the constant power load running at power level 5, which is calculated to be $1.13 \mathrm{~W}$ or $16.8 \mathrm{~V}$ for a $250 \Omega$ resistor. When another resistor of the same value was added to the load making it $125 \Omega$, the load voltage dropped to about $12 \mathrm{~V}$ and power was maintained at $1.13 \mathrm{~W}$. 


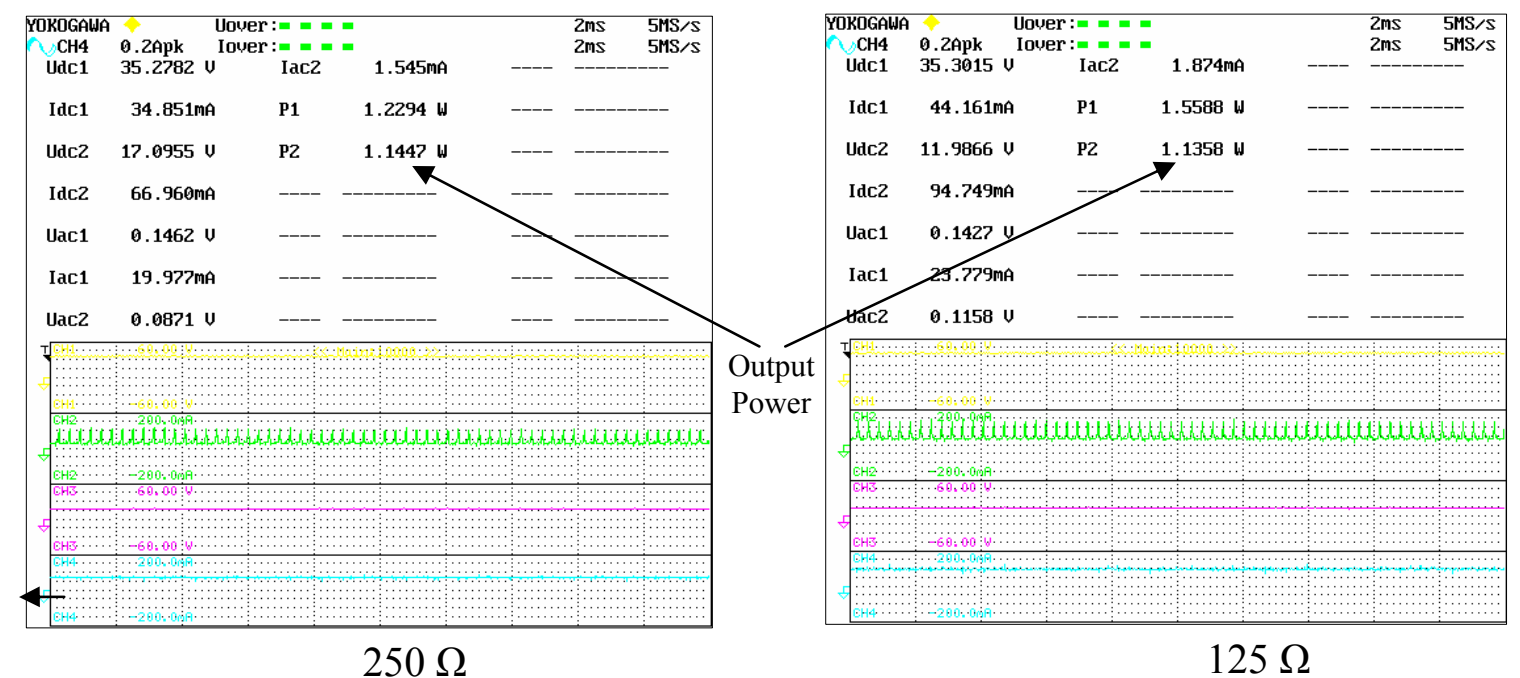

Figure 67: Constant Power Load with Lower Resistance. 


\section{Chapter 5 Conclusion}

\subsection{Conclusion}

This thesis presents the design of a testbed for the all-electric shipboard power system, which integrates power and communication in a single system. The testbed includes most of the loads present on a modern all electric shipboard. Communication is implemented using a single CAN bus. An external device can communicate with the local controllers to obtain measured values like voltage, current, and power demand or modify set-points. This hardware provides a platform for testing higher level distributed controls while it efficiently handles the embedded local controls and device safety. Overloading or short circuiting any terminals on the hardware is protected. All the controls and communication on the testbed operate in hard real-time. This easy to use hardware is ready to use right after power-up. Default set-point values are effective until they are altered using the remote terminal or other sources of IA messages. The testbed listens to valid commands on the CAN bus and takes actions as required.

The controls are implemented using programmable microcontrollers, making the testbed more flexible for upgrading or testing new control techniques for the individual devices.

The overall parts cost for the hardware was about $\$ 5300$. This number includes discrete components, circuit boards, microcontrollers, sensors, and relays. Another supporting expense of about \$ 2105 was made on MATLAB toolbox and additional materials to have 3-phase power available for the testbed.

\subsection{Future Work}

The testbed includes complete DC zonal distribution system, propulsion system, and pulse load making it a complete distribution testbed. The testbed could be connected to prime mover-generator sets to further evaluate dynamics during reconfiguration.

The testbed consists of at least three variable frequency motor drives. Currently these drives use a potentiometer at the MCU input for frequency adjustment. Speed controls 
through CAN interface can be added and integrated with the rest of the system. Magnetic speed sensors have been attached to each of these motors and the measured pulses are made available at the appropriate pins of the microcontroller. Closed loop controls can be established using the feedback loops.

Another upgrade to the testbed could be on the communication side. Although CAN is noise immune, it is still vulnerable in the sense that it requires a pair of twisted wires to be intact and to have communication available. Any disconnection in the network can bring the whole communication down. Encrypted wireless networks can be added via serial or SPI interface available with the existing MCU board to provide additional reliability. The existing microcontroller offers a program memory of $32 \mathrm{k}$ bytes and a data memory of $2 \mathrm{k}$ bytes. Although the compiled code for most of the controls for the project does not exceed $4 \mathrm{k}$ bytes in size, but some essential library files make the code size exceed 20k bytes in some cases. Therefore, the memory size may not be sufficient to implement the reconfiguration algorithm on the processor The processors could be upgraded to more powerful versions and this would provide enough resources to program the reconfiguration agents on the local devices themselves. 


\section{References}

[1] "The $I^{2} C$-bus Specification, Version 2.1, January 2000" available at http://www.nxp.com/acrobat_download/literature/9398/39340011.pdf

[2] Analog Devices, “ADSP-219x/2192 DSP Hardware Reference”, Revision 1.1, p. 567, April 2004.

[3] Axiom Manufacturing Inc., URL: http://www.axman.com/

[4] Balogh Laszlo, "Design And Application Guide For High Speed MOSFET Gate Drive Circuits", 2001 Unitrode Design Seminars - SEM1400, topic 2, Dallas, Texas, USA, 2001.

[5] Bell, C. G., Mudge, J. C., McNamara, J. E., Computer Engineering: A DEC View of Hardware Systems Design (1979), http://research.microsoft.com/users/gbell/Computer_Engineering/00000095.htm

[6] Benatmane, M., McCoy, T., "Development of a 19MW PWM Converter for US Navy Surface Ships," International Conference on Electric Machines / ElecShip '98, Istanbul, Turkey, September 1998.

[7] Bimal K. Bose, Modern Power Electronics and AC Drives, Prentice Hall PTR, Upper Saddle River, NJ 07458, 2002.

[8] CAN in Automation, URL: http://www.can-cia.org/canopen/

[9] Casini, Virgil, "Overview of Electrical Hazards", National Institute for Occupational Safety and Health (NIOSH), October 4, 2006.

[10] Crane, A., McCoy, T., "Electromagnetic Compatibility Design for a 19 MW PWM Motor Drive," IEEE IAS Annual Meeting, 3-7 October, 1999, Phoenix, AZ.

[11] Davis, Leroy, URL: http://www.interfacebus.com/Standards.html

[12] DeviceNet Introduction, Real Time Automation, URL: http://www.rtaautomation.com/devicenet/ 
[13] Elbanhawy, Alan, "Optimizing Voltage Selection in Buck Converters", Power Electronics Technology, June 2005.

[14] Embedded System Academy, "History of the $\mathrm{I}^{2} \mathrm{C}$ Bus", available at the URL: http://www.esacademy.com/

[15] Fairchild Semiconductor, H11L3M Opto-isolated NAND gate datasheet, December 2006

[16] Farsi, M. Ratcliff, K. Barbosa, M., "An overview of controller area network", Computing \& Control Engineering Journal, Aug 1999, Volume: 10, Issue: 3, p113-120

[17] Foundation for Intelligent Physical Agents, ACL Message Structure Specification, SC00061G, 2002.

[18] Foundation for Intelligent Physical Agents, FIPA ACL Message Representation in Bit-Efficient Encoding Specification, SC00069G, 2002.

[19] Freescale Semiconductor, "16-bit Microcontrollers MC9S12C32", Document Number: MC9S12C32FS, 2005.

[20] Freescale Semiconductor, "CodeWarrior Development Studio for HCS12(X) Microcontrollers", Data Sheet, 2007.

[21] Freescale Semiconductor's Corporate Website, URL: http://freescale.com/

[22] Hewlett-Packard, Tutorial Description of the Hewlett-Packard Interface BUS, HP184 November 1984.

[23] Hewlett Packard Technical Datasheet, "Low Input Current Logic Gate Optocouplers", Agilent Technologies, Retrieved from http://www.avagotech.com/assets/downloadDocument.do?id=21, on Nov 02, 2007.

[24] International Rectifier, IRAMS06UP60A datasheet, September, 2004, www.irf.com.

[25] Jayabalan, R.; Fahimi, B., "Naval shipboard power system", Vehicle Power and Propulsion, 2005 IEEE Conference. 
[26] Kolar, D.; Cerny, S., "Evolution of software for embedded system in processor expert", Proceedings of $11^{\text {th }}$ IEEE international Conference and Workshop on the Engineering of Computer Based Systems, 2004.

[27] LEM Components, Industrial Transducer Catalogue, Edition 2004/2005, September 2004.

[28] LEM USA, URL: http://www.lem.com/

[29] Luft, Lee A., Anderson, Larry, Frank Cassidy, "NMEA 2000® A Digital Interface for the $21^{\text {st }}$ Century", National Technical Meeting 2002, Institute of Navigation.

[30] Mathworks website, URL: http://www.mathworks.com/

[31] MATLAB, ver2006b help topic, abc_to_dq0 Transformaiton.

[32] MC9S12C Family Device User Guide V01.11, Freescale Semiconductor, 25 JAN 2003, Revised 28 FEB 2005

[33] Mccoy, T.J, “Trends in Ship Electric Propulsion," Panel Session at IEEE PES 2002 Summer Meeting.

[34] CodeWarrior Development Studio for Freescale HCS12(X) Microcontrollers data sheet.

[35] Mohan, Ned, Undeland, Tore, Robbins, Willam, Power Electronics Converters. Applications and Design, John Wiley \& Sons Inc, 1995, pp. 301, 16 and 23.

[36] MOST Cooperation, URL: http://www.mostcooperation.com/

[37] Nasser Kutkut, "High Efficiency Power Switches", Technical Note, Power Designers LLC, Retrieved on Nov 01, 2007 from http://www.powerdesigners.com/

[38] Nippon Chemi-Con, Miniature Aluminum Electrolytic Capacitors, Cat. No. E1001G (Ver.3), pp. 10.

[39] Nippon Chemi-Con, Miniature Aluminum Electrolytic Capacitors, KQM Series Datasheet. 
[40] Occupational Safety \& Health Administration (OSHA), "How Electrical Current Affects the Human Body", September 01, 2007.

[41] Omron Electronics datasheet on general-purpose MY family relays, Catalogue No. GC RLY7, Jan 2000.

[42] Pant, Pradeep, Schoder, Karl, Feliachi, Ali, "An Integrated Electric Shipboard Power System Testbed”, Electric Ship Technologies Symposium, 2007. ESTS'07. IEEE, 21-23 May 2007.

[43] Pekarek., S.D., Sudhoff, S.D., Sauer, J.D., Delisle, D.E., Zivi, E.J., "Overview of the Naval Combat Survivability Program", Proceedings of the Thirteenth Ship Control Systems Symposium (SCSS 2003), Orlando, Florida, April 7-9, 2003.

[44] Pinak Tulpule, "Multiagent Approach for Power System Reconfiguration", MS. Thesis, Lane Department of Computer Science and Electrical Engineering, West Virginia University, Morgantown, WV, 2007

[45] Press release on IEEE 488.1-2003, Available at URL: http://standards.ieee.org/announcements/pr_4881upgrade.html.

[46] Pressman, Abraham I., "Switching Power Supply Design", Second Edition, McGraw-Hill, 1998, pp 17, 359,

[47] Purdue University, Annual Research Summary 2003-2004, Electrical and Computer Engineering, URL: https://engineering.purdue.edu/ECE/Research/ARS/ARS2004.pdf

[48] Robert Bosch GmbH, "Bosch CAN Specifications, Version 2.0", 1991, URL: http://www.semiconductors.bosch.de/pdf/can2spec.pdf

[49] Robert C. Kremer \& Roberto A. Flores, "Using a Performative Subsumption Lattice to Support Commitment-based Conversations.", Proceedings of the 4th International Joint Conference on Autonomous Agents and Multi Agent Systems, ACM Press, pp. 114-121, Netherlands, July, 2005

[50] Ruff, Matthew, "Evolution of local interconnect network (LIN) solutions" Vehicular Technology Conference, 2003. VTC 2003-Fall. 2003 IEEE 58th.

[51] Sudhoff, S.D., Pekarek, S.D., Kuhn, B.T., Glover, S.F., Sauer, J. D., Delisle, E., "Naval Combat Survivability Testbeds for Investigation of Issues in Shipboard Power Electronics Based Power and Propulsion Systems", Proceedings of the 
IEEE Power Engineering Society Summer Meeting, July 21-25, 2002, Chicago, Illinois.

[52] Texas Instruments, Introduction To Controller Area Network, Application Report, August 2002.

[53] The MathWorks, Inc., Embedded Target for Motorola HC12 User's Guide, Version 1, February 2003

[54] The MathWorks, Inc., Target Language Compiler: Code Generation Process, Online Help available at URL: http://www.mathworks.com/access/helpdesk/help/toolbox/rtw/tlc/bp6j4co.html URL: http://www.interfacebus.com/Design_Connector_IEbus.html

[55] Valvano, Jonathan W, "Embedded Microcomputer Systems Real Time Interfacing", Second Edition, Thomson, 2007, pp. 314.

[56] Wikipedia, Local Interconnect Networking, URL: http://en.wikipedia.org/wiki/LIN

[57] Wunnava, S.V.; Hoo, P. Lexington, Kentucky, "Remote instrumentation access and control (RIAC) through inter-networking", IEEE Southeastcon Proceedings 1999. 


\section{Appendix A: Circuit Board and Wiring}

\section{Power Supply}

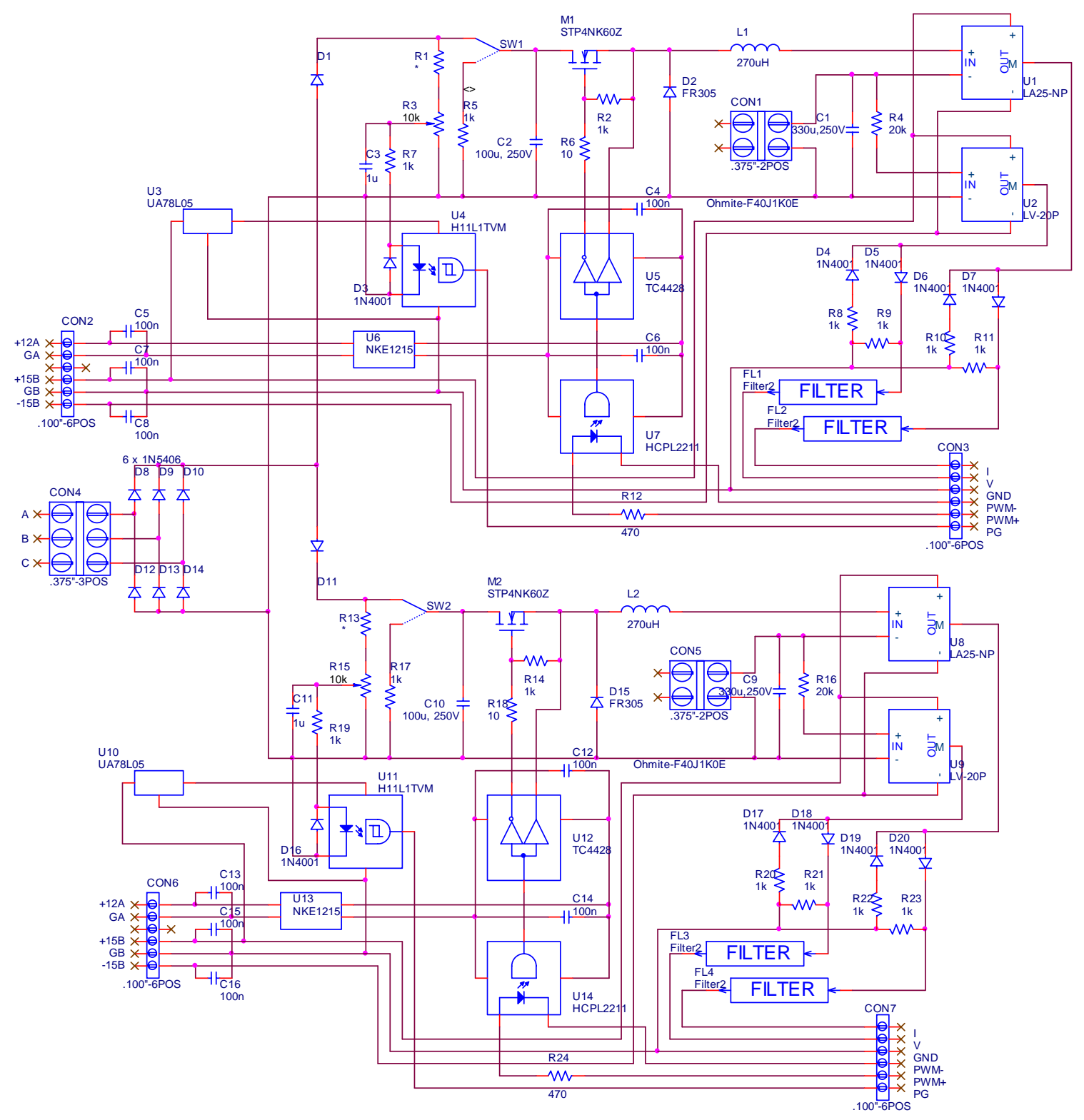

Figure 68: Power Supply Schematic. 


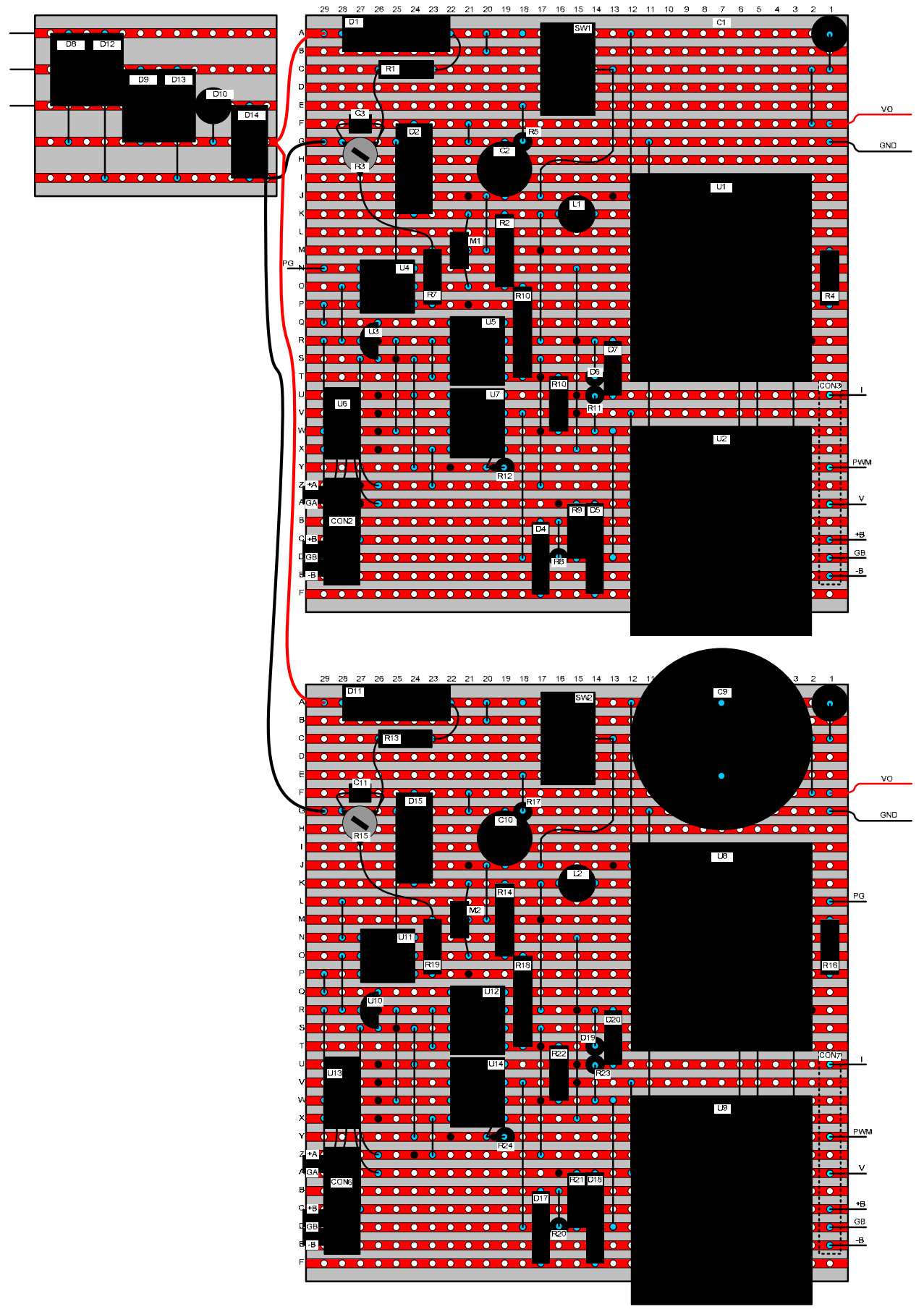

Figure 69: Power Supply Board and Wiring. 


\section{Motor Drive}

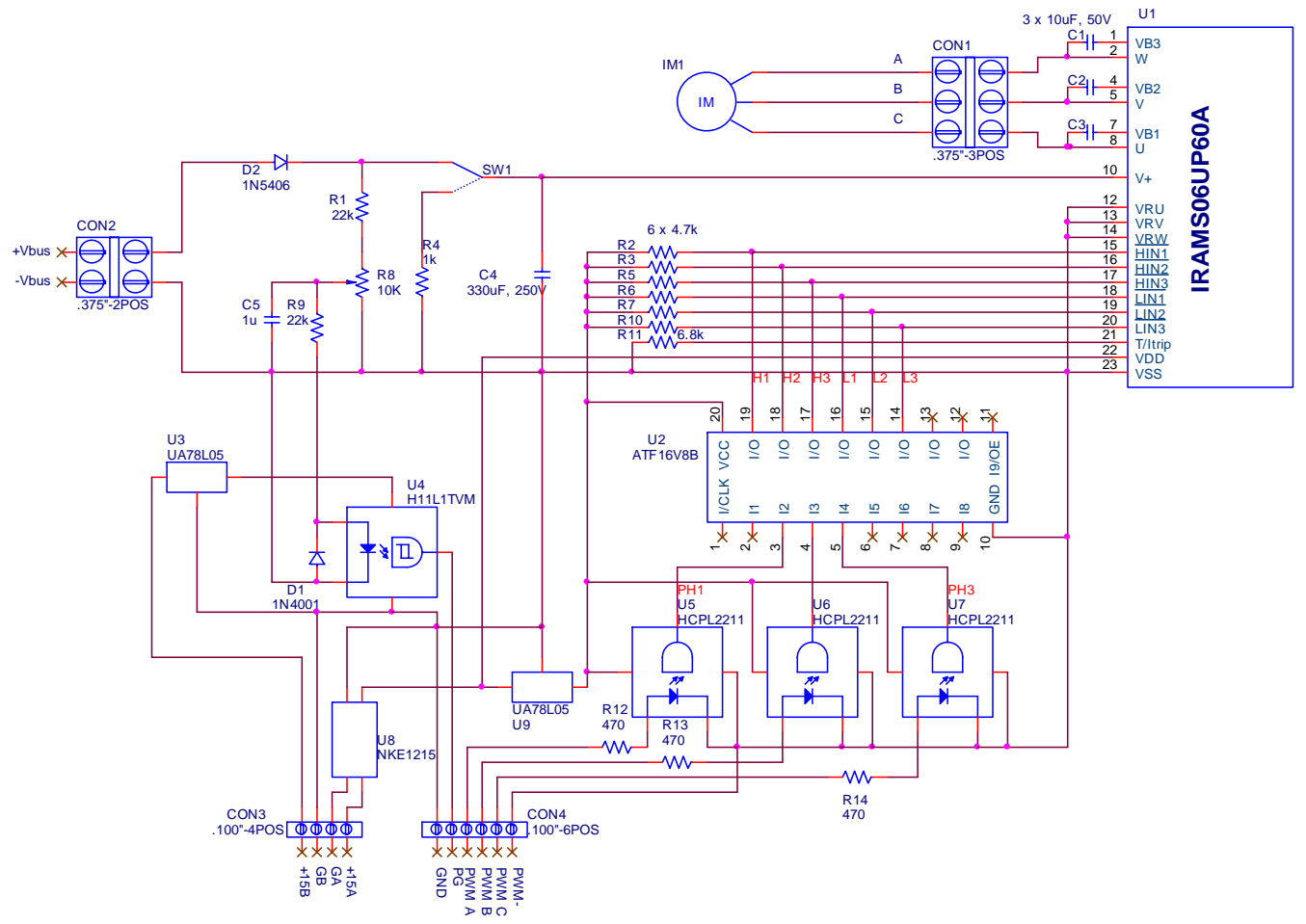

Figure 70: Motor Drive Schematic.

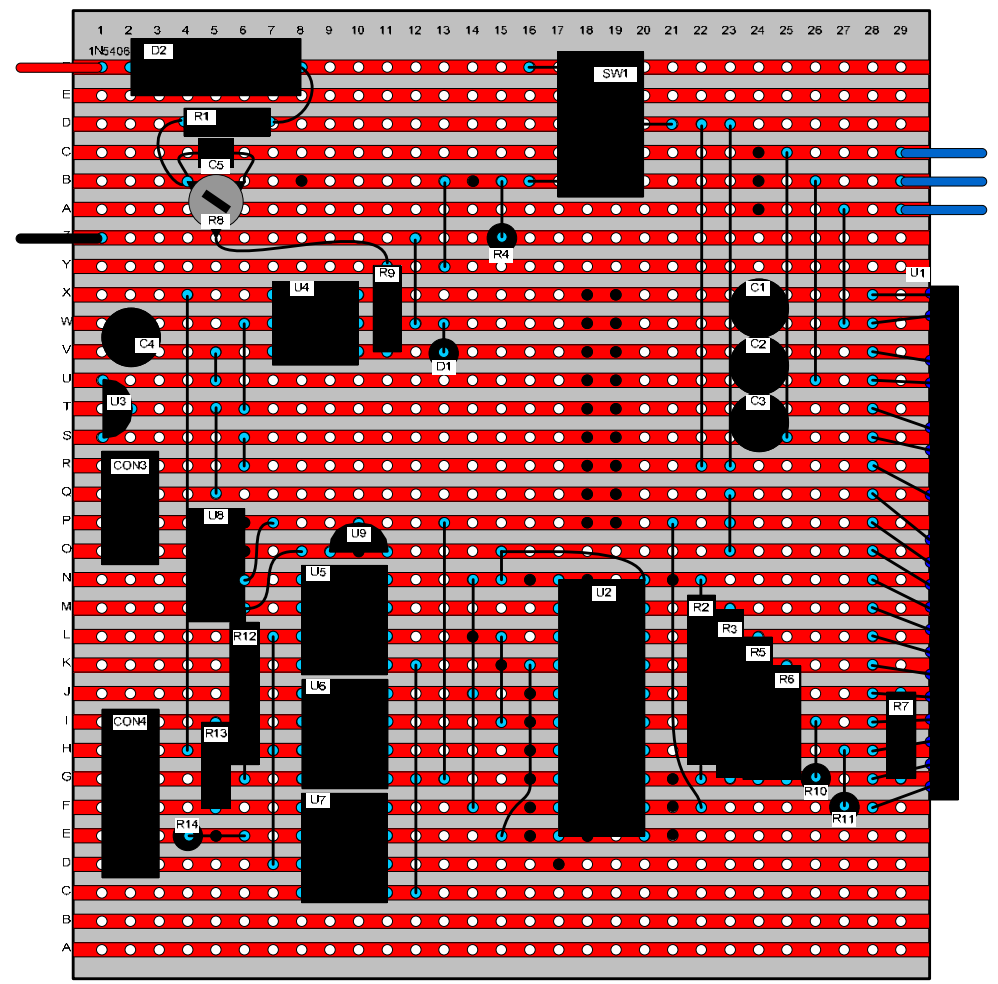

Figure 71: Motor Drive Board. 


\section{Pulsed Power Load}

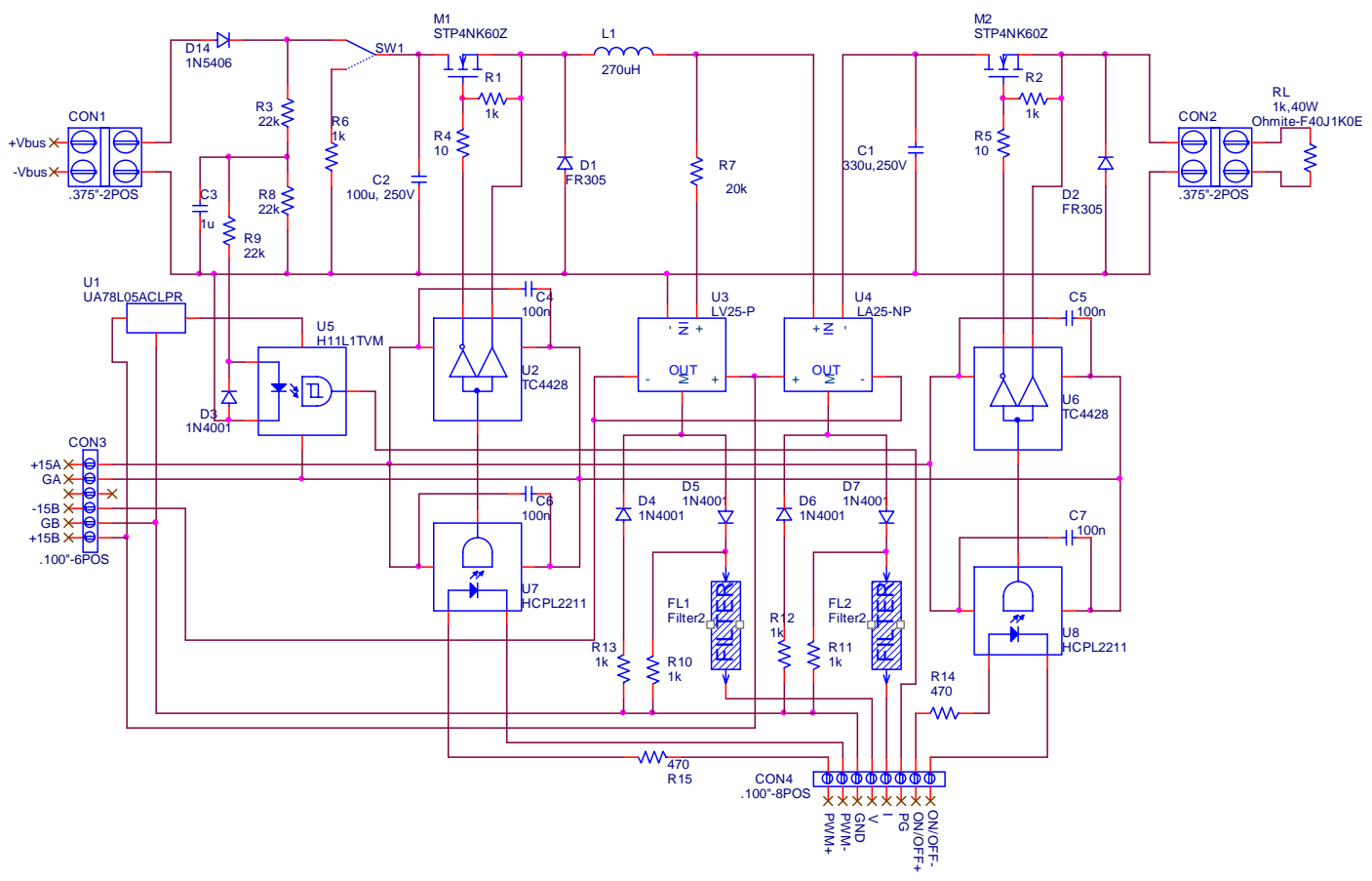

Figure 72: Pulsed Load Schematic.

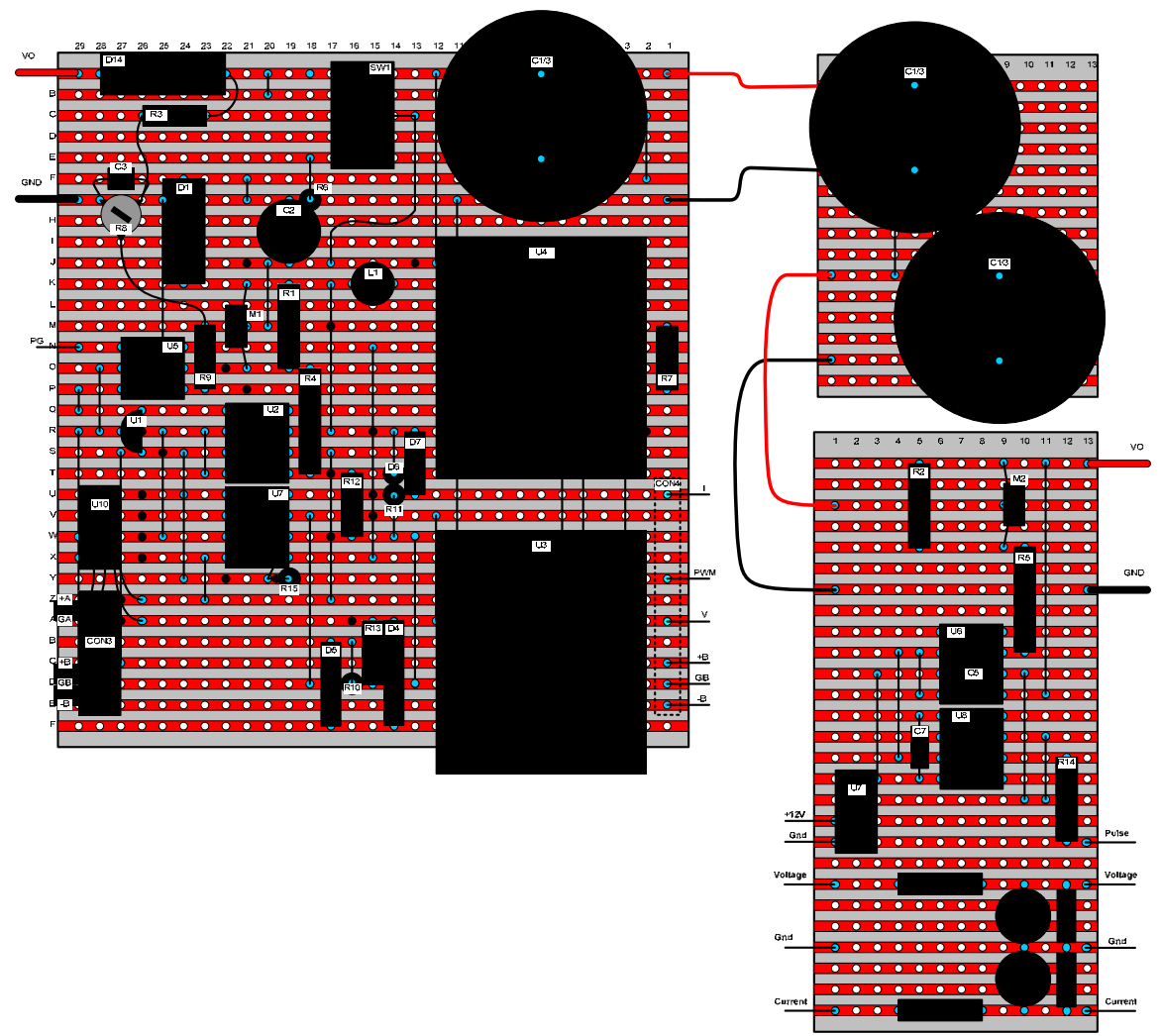

Figure 73: Pulsed Load Board and Wiring. 


\section{Shipboard Service Converter Module}

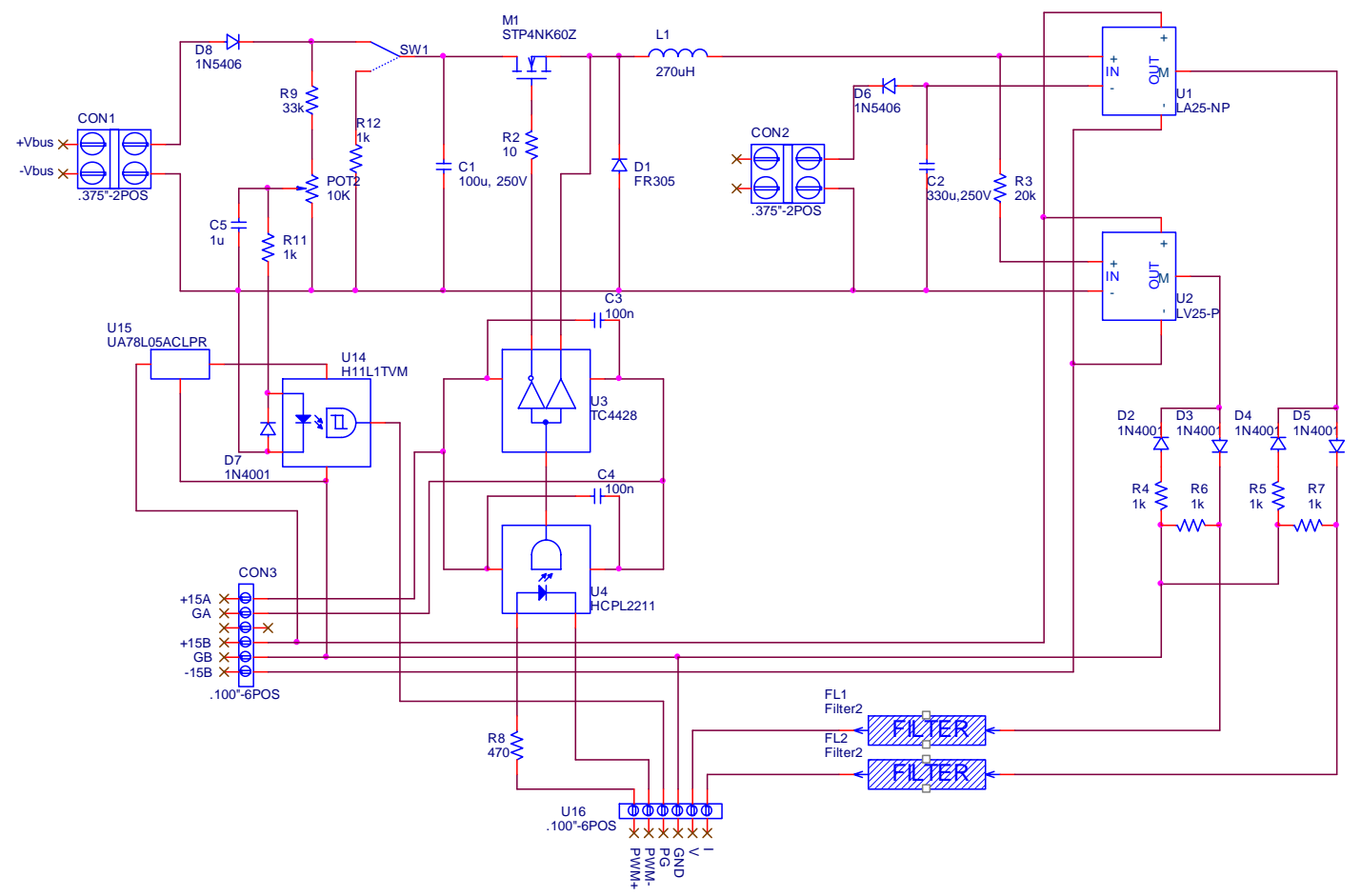

Figure 74: Shipboard Service Converter Module Schematic.

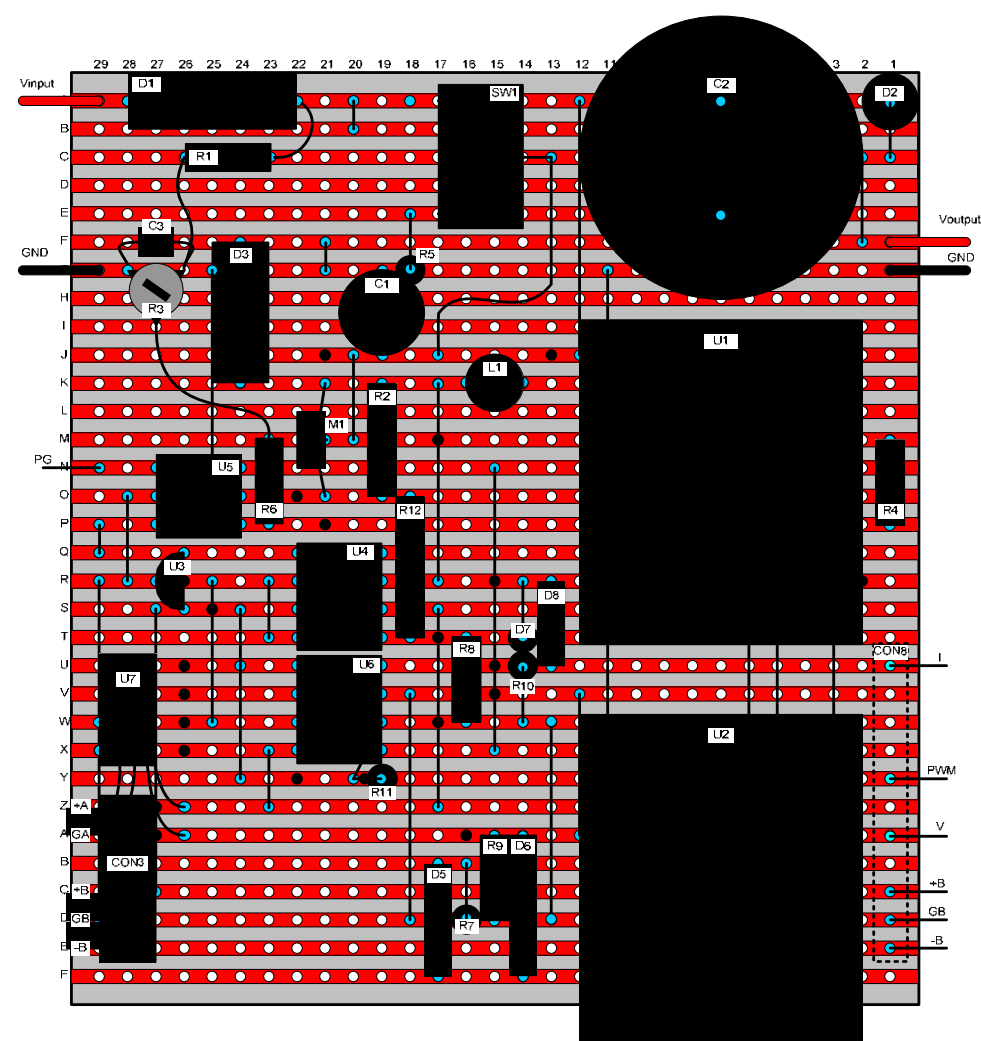

Figure 75: Shipboard Service Converter Module Board. 


\section{Constant Power Load}

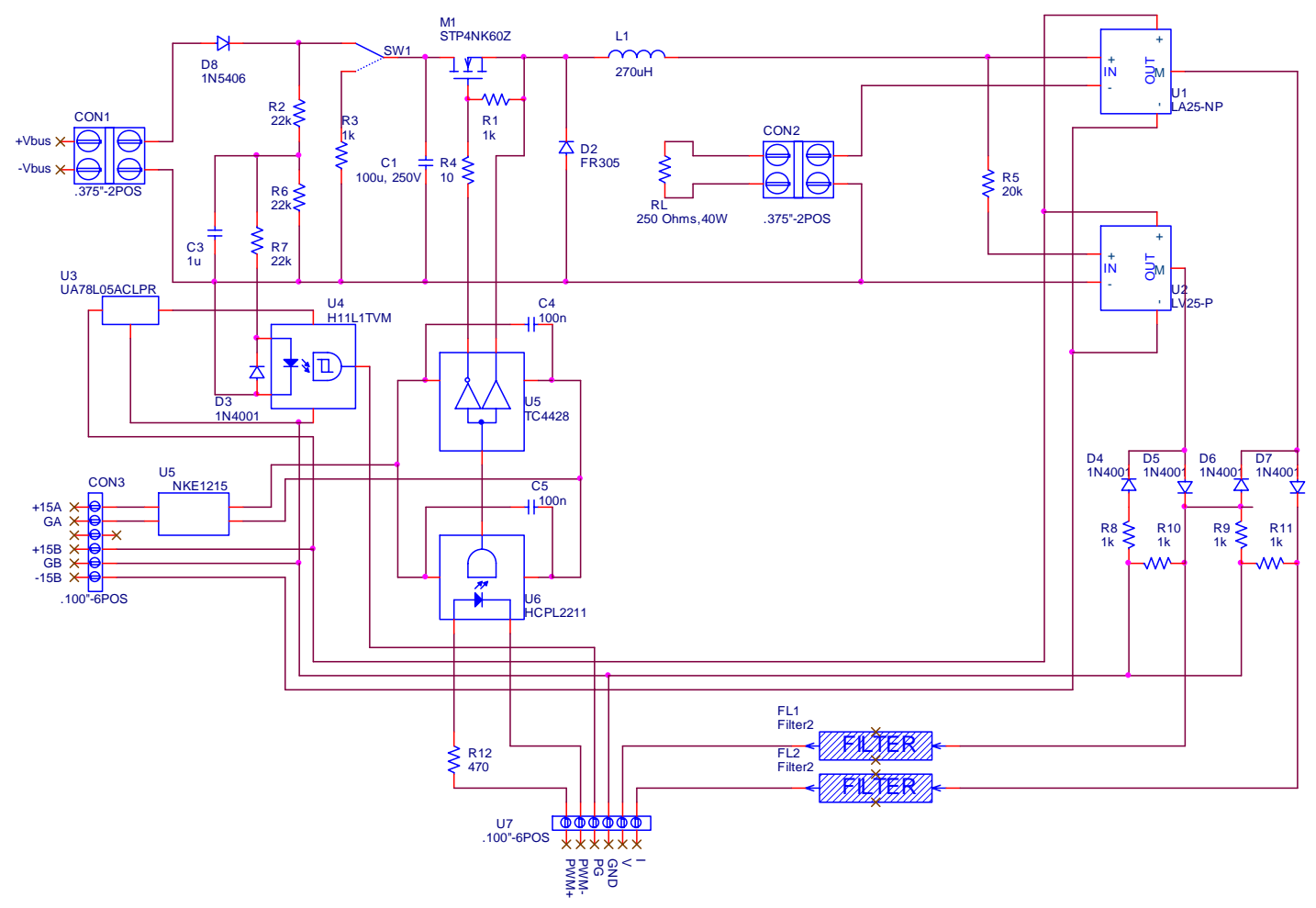

Figure 76: Constant Power Load Schematic.

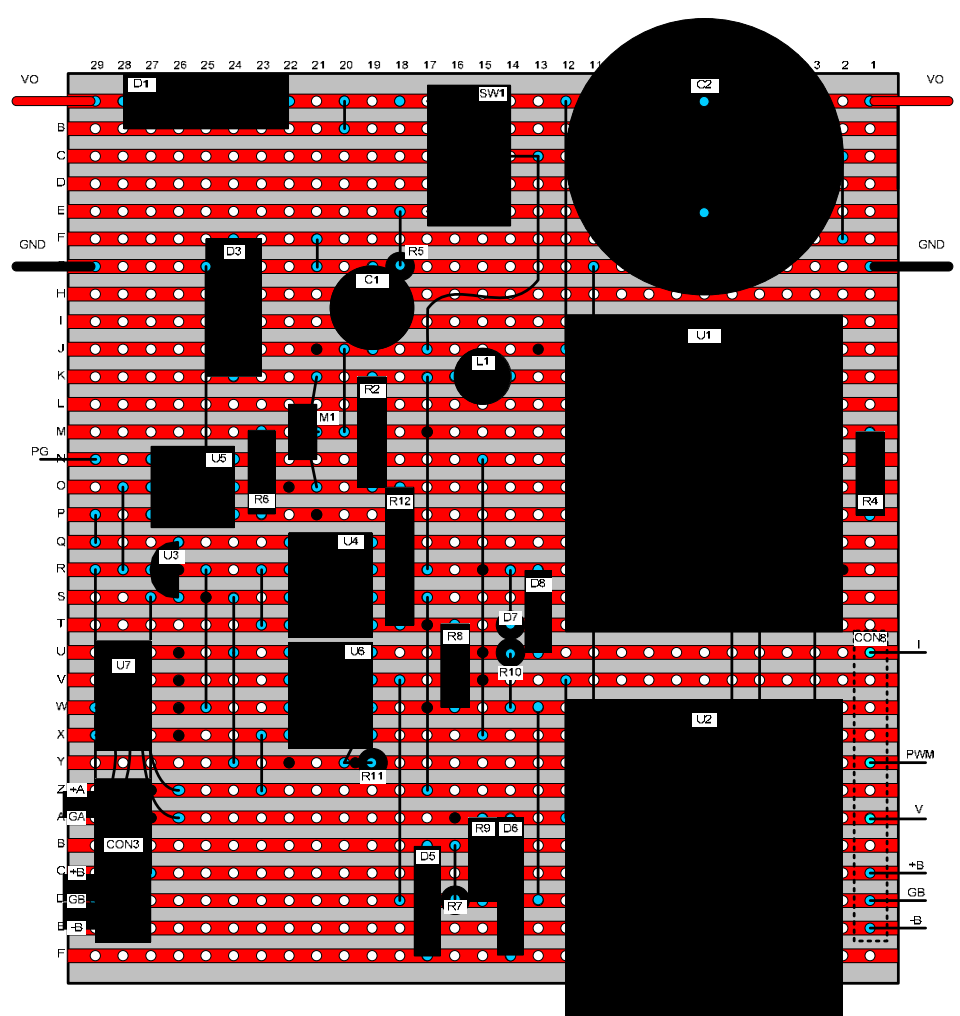

Figure 77: Constant Power Load Circuit Board. 